submitted to ApJ on 7 November 2008

\title{
Dust Processing and Grain Growth in Protoplanetary Disks in the Taurus-Auriga Star-Forming Region
}

\author{
B.A. Sargent ${ }^{1}$, W.J. Forrest ${ }^{2}$, C. Tayrien ${ }^{2}$, M.K. McClure ${ }^{3}$, Dan M. Watson ${ }^{2}$, G.C. Sloan ${ }^{4}$, \\ A. $\mathrm{Li}^{5}$, P. Manoj ${ }^{2}$, C.J. Bohac ${ }^{2}$, E. Furlan ${ }^{6}$, K.H. Kim², J.D. Green ${ }^{2}$
}

\begin{abstract}
Mid-infrared spectra of 65 T Tauri stars (TTS) taken with the Infrared Spectrograph (IRS) on board the Spitzer Space Telescope are modeled using populations of optically thin dust at two temperatures to probe the radial variation in dust composition in the uppermost layers of protoplanetary disks. Most spectra with narrow emission features associated with crystalline silicates require $\mathrm{Mg}$ rich minerals and silica, but a very small number suggest other components. Spectra indicating large amounts of enstatite at higher temperatures (400-500 K) also require crystalline silicates, either enstatite or forsterite, at temperatures lower $(100-200 \mathrm{~K})$ than those required for spectra showing high abundance of other crystalline silicates. A few spectra show $10 \mu \mathrm{m}$ complexes of very small equivalent width. They are fit well using abundant crystalline silicates but very few large grains, inconsistent with the expectation that low peak-to-continuum ratio of the $10 \mu \mathrm{m}$ complex always indicates grain growth. Most spectra in our sample are fit well without using the opacities of large crystalline silicate grains. If large grains grow by agglomeration of submicron grains of all dust types, the amorphous silicate components of these aggregates must typically be more abundant than the crystalline silicate components. We also find that the more there is of one crystalline dust species, the more there is of the others. This suggests
\end{abstract}

\footnotetext{
${ }^{1}$ Space Telescope Science Institute, 3700 San Martin Drive, Baltimore, MD 21218; sargent@stsci.edu

${ }^{2}$ Department of Physics and Astronomy, University of Rochester, Rochester, NY 14627

${ }^{3}$ Department of Astronomy, The University of Michigan, 830 Dennison Building, 500 Church Street, Ann Arbor, MI, 48109-1042

${ }^{4}$ Center for Radiophysics and Space Research, Cornell University, Ithaca, NY 14853

${ }^{5}$ Department of Physics and Astronomy, University of Missouri, Columbia, MO 65211

${ }^{6}$ Spitzer Fellow; JPL, Caltech, Mail Stop 264-767, 4800 Oak Grove Drive, Pasadena, CA 91109
} 
that crystalline silicates are processed directly from amorphous silicates, whether by evaporation of the amorphous grains and condensation in chemical equilibrium or by annealing of the amorphous precursor grains. This also suggests that neither forsterite, enstatite, nor silica are intermediate steps along the way to producing either of the other two for the majority of the crystalline dust produced. Crystalline silicate abundance is correlated tightly with disk geometry, in the sense of higher crystallinity accompanying more settled disks, which are commonly associated with growth and settling of grains. Large-grain abundance is also correlated with disks that are more highly settled, but with a wide range of large grain abundance for a given degree of settling. We interpret this range to mean that the settling of large grains is sensitive to individual disk properties. We also find that lower-mass stars have higher abundances of large grains in their inner regions.

Subject headings: circumstellar matter, infrared: stars, stars: pre-main-sequence, planetary systems: protoplanetary disks

\section{Introduction}

Comets are thought to be largely unaltered reservoirs of leftover material from the primordial mixture of dust in the Solar Nebula orbiting the newly-formed Sun (e.g., Wooden et al. 2005). Spectral observations of Comet Hale-Bopp by Crovisier et al. (1997) using the Infrared Space Observatory (ISO; Kessler et al. 1996) allowed for detailed spectral models over a wide range of wavelengths ( $\mathrm{Li} \&$ Greenberg 1998; Wooden et al. 1999; Harker et al. 2002). The amorphous dust of pyroxene $\left([\mathrm{Mg}, \mathrm{Fe}] \mathrm{SiO}_{3}\right)$ or olivine $\left([\mathrm{Mg}, \mathrm{Fe}]_{2} \mathrm{SiO}_{4}\right)$ stoichiometry (henceforth, "amorphous pyroxene" or "amorphous olivine", respectively) was found to require a stoichiometric ratio of magnesium to iron of about unity, being relatively iron-rich (Harker et al. 2002); the crystalline pyroxene must be iron-poor, as it is cooler (Wooden et al. 1999); and the larger grains must be porous, fluffy aggregates of smaller grains (Li \& Greenberg 1998; Harker et al. 2002). Interplanetary Dust Particles (IDPs; Bradley 2003), believed to originate from comets, were often found to be highly porous aggregates of Mg-rich crystalline silicate dust, having qualities very similar to those inferred from comet spectra and spectra of Young Stellar Objects (YSOs; systems in the process of forming stars and planets).

Using increasingly powerful telescopes and sensitive detectors, astrophysicists have obtained increasingly higher-quality spectra for decreasingly fainter objects. Cohen \& Witteborn (1985) distinguished $10 \mu \mathrm{m}$ silicate complexes in absorption versus emission, while Honda et al. 
(2003) identified amorphous silicates, forsterite, enstatite, and silica using their spectrum of the $10 \mu \mathrm{m}$ complex of Hen 3-600A.

Approaches toward analysis of the dust composition of protoplanetary disks in Herbig Ae/Be and T Tauri (SED Class II YSO) systems have taken two forms: computation of indices from flux ratios over the $10 \mu \mathrm{m}$ (and sometimes longer wavelength) complex, or detailed modeling using opacities of minerals and amorphous dust measured on Earth. Studies taking the former approach include Bouwman et al. (2001), Przvgodda et al. (2003), van Boekel et al. (2003), Kessler-Silacci et al. (2005), Kessler-Silacci et al. (2006), and Watson et al. (2008). Studies taking the latter approach include Bouwman et al. (2001), Bowev \& Adamson (2002), Molster et al. (2002), Li \& Lunine (2003a), Li \& Lunine (2003b), Li et al. (2003), Bouwman et al. (2003), van Boekel et al. (2004), Uchida et al. (2004), van Boekel et al. (2005), Schütz et al. (2005), Sargent et al. (2006), Schegerer et al. (2006), Honda et al. (2006), and Sargent et al. (2008).

The Infrared Spectrograph (IRS; Houck et al. 2004) on board the Spitzer Space Telescope (Werner et al. 2004) allows good S/N spectra to be obtained at 5-37 $\mu \mathrm{m}$ wavelength for objects with flux densities of a few tens of mJy. Dust at a considerable range of temperatures in protoplanetary disks gives rise to the emission seen from such systems in IRS spectra. Our approach has been to model this using two temperatures (see Chen et al. 2006; Kastner et al. 2006; Sargent et al. 2008), though others have used more temperatures (see Bouwman et al. 2008). Here we model the dust emission over the 7.7-37 $\mu \mathrm{m}$ wavelength range for 65 Class II YSOs in the 1-2 Myr old Taurus-Auriga star-forming region (Kenyon \& Hartmann 1995) using dust at two temperatures, in order to analyze the dust composition in the inner and outer disk regions.

"Crystalline indices" computed from flux ratios that characterize crystalline silicate emission have the advantage of being quickly computed and measured repeatably for a wide range of objects. However, they do not make full use of the information on dust emissivity available in a high-quality mid-infrared spectrum; for instance, they measure the combined effect of grain growth and crystallinity, but such are difficult to disentangle (Watson et al. 2008) without detailed modeling using dust opacities, as is done here.

\section{Data Reduction}

Much of the process of data reduction of the 65 spectra analyzed in this study has been described by Sargent et al. (2008) and Furlan et al. (2006). Here we give a brief overview and provide details specific to this study. We note that the spectra of ZZ Tau and ROXs 42C 
used here are exactly the same as used and described by Sargent et al. (2008); the following discussion of data reduction applies to all spectra except those of ZZ Tau and ROXs 42C.

\subsection{Observations}

Sixty-three of the 65 objects in our sample come from the 85 Class II Young Stellar Objects (YSOs) analyzed by Furlan et al. (2006). The other 22 objects whose spectra were analyzed by Furlan et al. (2006) were rejected from our study due to high (here, defined as having $A_{V}>6$ ) or unknown extinction, the presence of Polycyclic Aromatic Hydrocarbon bands, saturation of the detectors, and confusion of Class II YSOs with Class I YSOs in the same spectrograph slit. To the 63 from Furlan et al. (2006) we add GG Tau B and Haro 6-28. For opacity calibration (see Section 3), we reduced data for ROXs 42C (see Sargent et al. (2008)), HBC 656, and TW Cha. All of these objects were observed using the combinations of IRS modules Short-Low and Long-Low or Short-Low, Short-High, and Long-High in either Staring Mode or Mapping Mode; for more information, see Sargent et al. (2008).

\subsection{Extraction and Calibration of Spectra and Rebinning of High Resolution Spectra}

We obtained Basic Calibrated Data products from the Spitzer Science Center (SSC) for each of the targets in our sample. BCD data are corrected for the dark current, stray light, and flatfield variations. The spectrum of HBC 656 was reduced using data from the S16.1.0 data calibration pipeline. The spectrum of ZZ Tau was reduced using data from the S14.0.0 IRS data calibration pipeline, using the methods described by Sargent et al. (2008); the present discussion of extraction and calibration of spectra excludes the spectrum of ZZ Tau. For the spectra of TW Cha and the $64 \mathrm{~T}$ Tauri stars in Taurus-Auriga excluding ZZ Tau, the data are from the S13.2.0 data calibration pipeline.

Next we identified and fixed by interpolation in the spectral direction bad pixels, as described by Watson et al. (2007) and Sargent et al. (2008). We then extracted spectra using the Spectral Modeling, Analysis, and Reduction Tool (SMART; Higdon et al. 2004). We sky-subtracted data from the low spectral resolution modules SL and LL using the off-order observation. We did not subtract sky from high spectral resolution ( $\mathrm{SH}$ and $\mathrm{LH}$ ) observations, as explained by Sargent et al. (2006). Multiple Data Collection Events (DCEs; detector samplings) at the same telecope position, same order, and same modules were averaged, and the uncertainties were calculated from the standard deviation of the mean. Tapered column 
extraction was used for SL and LL data, and full-column extraction was used for SH and LH data (Sargent et al. 2006). We used Relative Spectral Response Functions (RSRFs) to calibrate the flux (Sargent et al. 2006). For both orders of SL and for the both orders of LL (for all observations except for HBC 656), a spectral template of $\alpha$ Lacertae (A1 V; M. Cohen 2004, private communication) of higher spectral resolution than the templates described by Cohen et al. (2003) was used. For the LL flux calibration of HBC 656 over second, bonus, and first orders out to $36 \mu \mathrm{m}$ wavelength, RSRFs generated from data of $\xi$ Dra and the template for $\xi$ Dra from Cohen et al. (2003) were used. Past $36 \mu \mathrm{m}$, a LL first-order RSRF generated from data of Markarian 231 and the template for Markarian 231 (J. Marshall, private communication; Marshall et al. 2007; Armus et al. 2007) were used. For SH and LH, the spectral template for $\xi$ Dra (K2 III) by Cohen et al. (2003) and its IRS data were used to generate RSRFs. Except for GG Tau A, GG Tau B, ZZ Tau, ROXs 42C, HBC 656, and TW Cha, all RSRF-calibrated spectra are the same as presented by Watson et al. (2008).

We rebin our high-resolution spectra (see Table A1 for whether the spectra were obtained with high or low spectral resolution) to low spectral resolution. The width of each bin is determined by requiring spectral resolution $\mathrm{R}=\lambda / \Delta \lambda \sim 90$, and rebinned spectrum is sampled such that each bin is $\Delta \lambda$ from the next bin.

\subsection{Correction for Extinction, Mispointing, Uncertainties, and Stellar Photosphere}

We corrected our spectra for extinction using the same method described by Sargent et al. (2006), except we assume a ratio of V-band extinction to total optical depth at $9.7 \mu \mathrm{m}$, $\mathrm{A}_{V} / \tau_{9.7}$ of 25 . This value is suggested by data plotted by Chiar et al. (2007). We use values of $\mathrm{A}_{V}$ given by Furlan et al. (2006). We do not correct for extinction if $\mathrm{A}_{V}<1.4$, as we want to minimize any artifacts due to overcorrection of extinction (see Sargent et al. 2006). CoKu Tau/4, GG Tau A, GG Tau B, and Haro 6-28 are not corrected, but V410 Anon 13 is, assuming $\mathrm{A}_{V}=5.8$ (see Sargent et al. 2006). The extinction correction for ROXs $42 \mathrm{C}$ is the same as made by Sargent et al. (2008). The average (V-R) colors as measured by Batalha et al. (1998) of HBC 656 (also known as AS 216) are $\sim 0.64$, which is slightly bluer than the intrinsic (V-R) color of a K2 star (the spectral type assigned to HBC 656 by Batalha et al. 1998) of 0.74 given by Kenyon \& Hartmann (1995). This is consistent with HBC 656 being of slightly earlier spectral type (e.g., K0) and having $\mathrm{A}_{V} \sim 0$, so we assign $\mathrm{A}_{V}=0$ to HBC 656. Finally, we compute $\mathrm{A}_{V}=1.78$ for TW Cha using I and J magnitudes from DENIS (Cambresv et al. 1998) and intrinsic colors for a K4 main-sequence star from Kenyon \& Hartmann (1995), assigning a spectral type of K4 to TW Cha despite Luhman 
(2004) adopting K8 for TW Cha after having found various classifications for this star in the literature of $\mathrm{K} 0, \mathrm{~K} 8$, and $\mathrm{M} 0$.

Next, we corrected for slight mispointing of the telescope from the standard nod positions by scaling the mispointed nods, as described by Sargent et al. (2008). The scalars used are given in Table A1 and further described in the Appendix. After applying these scalars, the spectral uncertainties were computed. For all objects except ZZ Tau, the flux density uncertainties were half the difference between the spectra obtained at the two nod positions (see Sargent et al. 2006). The uncertainties were derived from only two independent measurements of flux (except for ZZ Tau), so sometimes the relative uncertainty was less than $1 \%$. Because of this and because of uncontrolled systematic errors, when the relative uncertainty was less than 1\%, it was set to $1 \%$ as described by Sargent et al. (2008). The spectra plotted by Furlan et al. (2006) for CoKu Tau/4, DM Tau, and GM Aur showed very little excess emission above the stellar photosphere at $\lambda<8 \mu \mathrm{m}$; for these, we subtracted Planck functions using solid angles and effective stellar temperatures given by Sargent et al. (2006) for the same purpose. Photospheres were subtracted for ZZ Tau and ROXs 42C in exactly the manner described by Sargent et al. (2008). For all other spectra, the stellar photosphere contribution to the spectra appears minor, and stellar photospheres are not subtracted before modeling them.

\section{Analysis}

\section{1. $\quad$ Models}

To characterize the silicate dust composition of the upper levels of the T Tauri disks giving rise to the emission features in our spectra, we construct detailed models (Sargent et al. 2008). Briefly summarized, each model is a sum of emission from blackbodies at temperatures $\mathrm{T}_{w}($ warm $)$ and $\mathrm{T}_{c}(\mathrm{cool})$ and emission from optically thin dust grains proportional to their opacities multiplied by Planck functions at $\mathrm{T}_{w}$ and $\mathrm{T}_{c}$ :

$$
\begin{aligned}
F_{\nu}(\lambda)^{\bmod }= & B_{\nu}\left(\lambda, T_{c}\right)\left[\Omega_{c}+\sum_{i} a_{c, i} \kappa_{i}(\lambda)\right]+ \\
& B_{\nu}\left(\lambda, T_{w}\right)\left[\Omega_{w}+\sum_{j} a_{w, j} \kappa_{j}(\lambda)\right],
\end{aligned}
$$

where $\mathrm{B}_{\nu}(\lambda, \mathrm{T})$ is the Planck function evaluated at wavelength $\lambda$ and temperature $\mathrm{T}, \Omega_{c}\left(\Omega_{w}\right)$ 
is the solid angle of the blackbody at temperature $\mathrm{T}_{c}\left(\mathrm{~T}_{w}\right), \mathrm{a}_{c, i}\left(\mathrm{a}_{w, i}\right)$ is the mass weight of component $\mathrm{j}$ at temperature $\mathrm{T}_{c}\left(\mathrm{~T}_{w}\right)$, and $\kappa_{j}(\lambda)$ is the opacity (effective cross-section per unit mass) at wavelength $\lambda$ of dust species $\mathrm{j}$. The mass weights, $\mathrm{a}_{c, i}\left(\mathrm{a}_{w, i}\right)$, are equal to $m_{c, i} / d^{2}$ $\left(m_{w, i} / d^{2}\right)$, where $m_{c, i}\left(m_{w, j}\right)$ is the mass of dust species $i$ at $T_{c}\left(T_{w}\right)$ and $d$ is the distance to the T Tauri star, which is assumed to be 140 parsecs for T Tauri stars in the TaurusAuriga star-forming region (Kenyon et al. 1994). The blackbody components of each model, $\Omega_{c} \mathrm{~B}_{\nu}\left(\lambda, \mathrm{T}_{c}\right)$ and $\Omega_{w} \mathrm{~B}_{\nu}\left(\lambda, \mathrm{T}_{w}\right)$, represent optically thick continuum emission, unsubtracted stellar photosphere emission $\left(\mathrm{F}_{\nu}\right.$ proportional to $1 / \lambda^{2}$, and it should be small for spectra for which we have not already subtracted a Planck function at the stellar effective temperature to represent it), and emission from grains in the optically thin disk regions whose opacities are featureless continua, like amorphous carbon and very large silicate grains. The model components proportional to opacity multiplied by a Planck function represent emission from submicron- to few-micron-sized dust grains, located in the optically thin disk regions, with strong resonances giving rise to detectable features in their opacities.

We will assume in the following that all grain species in the same location in the disk have the same temperature, the thermal coupling provided by gas. Kamp \& Dullemond (2004) find that the layer with $\mathrm{A}_{V} \leq 0.1$ (where $\mathrm{A}_{V}$ is the visual extinction of star into the disk), the layer above the layer where dust emission features arise (what they call the "superheated surface layer"), has gas and dust temperatures within $10 \%$ of each other, suggesting the gas would bring dust grains of differing optical properties to roughly the same temperature. In general, settling of dust towards the disk midplane (D'Alessio et al. 2006) should enhance the gas particle density for the optically thin regions in the disk atmosphere that gives rise to the $10 \mu \mathrm{m}$ silicate feature because the gas density is higher towards the disk midplane (see Figure 1 of Glassgold et al. 2004), so dust and gas temperatures should be better coupled in more settled disks. However, Chiang \& Goldreich (1997) found that, for a fiducial disk at 1 $\mathrm{AU}$, the dust grains should remain at their radiative equilibrium temperatures, undisturbed by gas-grain collisions, for the vast majority of plausible gas particle densities, $10^{8}$ to $3 \times 10^{14}$ $\mathrm{cm}^{-3}$, at this distance from the star. They found that only between about $10^{14}$ and $3 \times 10^{14}$ $\mathrm{cm}^{-3}$ would the dust grain temperatures be affected by collisions with gas molecules. At 8 AU, Gorti \& Hollenbach (2008) find that gas and dust temperatures are only equal for $\mathrm{A}_{V}$ slightly greater than 1, suggesting greater difference between dust and gas temperature in the "superheated surface layer" than Kamp \& Dullemond (2004) predict. For the purposes of dust modeling of T Tauri stars, Planck functions and opacity-weighted Planck functions at two temperatures (at most) will be used, while keeping in mind the results of these sophisticated studies of dust and gas coupling. We allow for the two temperatures in order to study compositional differences of disks between inner disk regions and outer disk regions. If, however, there is not sufficient coupling of dust and gas heating in the optically thin 
surface layers giving rise to emission features in our TTS spectra, this means dust that absorbs starlight poorly at optical and near-infrared wavelengths with respect to mid-infrared wavelengths, like enstatite and forsterite, will be located closer to the star than dust like ironrich amorphous pyroxene and olivine at the same temperature. Such iron-rich amorphous silicates absorb starlight more efficiently at visible and near-infrared wavelengths with respect to mid-infrared wavelengths (Harker et al. 2002).

To determine the best fit of a model to a spectrum, we minimize $\chi^{2}$ between the model and the 7.7-37 $\mu \mathrm{m}$ region of the spectrum, with respect to the two temperatures, the solid angles, and the mass weights. We choose ranges of $\mathrm{T}_{c}$ and $\mathrm{T}_{w}$ to explore, and, picking a single pair of $\left[\mathrm{T}_{c}, \mathrm{~T}_{w}\right]$, we minimize $\chi^{2}$,

$$
\chi^{2}=\sum_{k}\left[\frac{F_{\nu}\left(\lambda_{k}\right)^{\mathrm{irs}}-F_{\nu}\left(\lambda_{k}\right)^{\mathrm{mod}}}{\Delta F_{\nu}\left(\lambda_{k}\right)^{\mathrm{irs}}}\right]^{2},
$$

with respect to all solid angles and all mass weights. When this results in mass weights or solid angles that are negative, we eliminate from the model the component whose flux integrated from 7.7-37 $\mu \mathrm{m}$ is most negative, and minimize $\chi^{2}$ of the model without this component. This is repeated at a given temperature pair $\left[\mathrm{T}_{c}, \mathrm{~T}_{w}\right]$ until there are no negative mass weights 1 . This entire process is carried out for each temperature pair. The temperature pair for which $\chi^{2}$ per degree of freedom (where the number of degrees of freedom is the number of data points $(\sim 200)$ minus the number of temperatures used, 2 , minus the number of nonzero solid angles minus the number of nonzero mass weights) is lowest is the best-fit model for a spectrum.

We also determine uncertainties for our model parameters. We define the uncertainty in a parameter to be the increment of the parameter from its best-fit model value that changes by 1.0 the value of $\chi^{2}$ per degree of freedom, or $\chi_{\nu}^{2}$, of the model with the incremented value of the parameter. We use a Taylor series expansion to approximate this.

We note that our definition of uncertainty in a parameter is similar to what Wooden et al. (1999) call "independent point uncertainty" in that all model parameters are kept at their best-fit values except for the one whose uncertainty is being determined. However, our uncertainties are determined differently from how others have been determined in the literature. Press et al. (1992) suggest taking the uncertainty to be that increment of the parameter

\footnotetext{
${ }^{1}$ The only solid angle that was found to be negative and in the subsequent iteration zeroed out was that of the cool blackbody component of $\mathrm{CoKu} \mathrm{Tau} / 3$
} 
that increases $\chi^{2}$ by a number computed from the incomplete gamma function for a given level of significance. Avni (1976) found that uncertainties computed using the increment in $\chi^{2}$ determined in this manner are quite consistent with uncertainties computed from Monte Carlo analysis. In such an analysis, the standard deviation in the set of parameter values obtained by fitting multiple versions of the same spectrum, each manipulated by adding noise according to Poisson statistics, is taken to be the uncertainty in that parameter.

For example, the model for DG Tau has only eight constraints. The incomplete gamma function implies that an increment of $\chi^{2}$ of 9.31 would determine the $68.3 \%$ (one sigma) uncertainty level on parameters. This would result in a one sigma uncertainty on the mass weight of cool small amorphous pyroxene of $1.68 \times 10^{-18} \frac{\mathrm{gm}}{\mathrm{cm}^{2}}$. This is nearly identical to the uncertainty on this component obtained via the Monte Carlo analysis described previously of $1.62 \times 10^{-18} \frac{\mathrm{gm}}{\mathrm{cm}^{2}}$. Using the method adopted in this study, an uncertainty of $8.21 \times 10^{-18}$ $\frac{\mathrm{gm}}{\mathrm{cm}^{2}}$ is obtained, which is almost 5 times higher than the one sigma uncertainties obtained using the incomplete gamma function to determine the increment of $\chi^{2}$ or the Monte Carlo approach. We note that, since the number of data points (about 200) in one of our spectra is much greater than the number of model constraints (by a factor of $>20$ ), our uncertainty

level involves increasing $\chi^{2}$ by much more than suggested by Press et al. (1992). By so doing, we conservatively bias the uncertainties in our parameter values to many times one sigma.

Our models include the opacity of annealed silica from Fabian et al. (2000). This was shown to provide the best fit to emission features at $9,12,16$, and $20 \mu \mathrm{m}$ in astronomical spectra of TTS by Sargent et al. (2008). We now justify our choice of opacities for submicron amorphous and crystalline silicates and large (few-micron-sized) silicate dust grains. In order to choose the best opacity curves to use to model emission from TTS, we assume that all disks in our sample have the same major kinds of dust, but in different relative abundances. Assuming all disks have basically the same major kinds of dust - amorphous silicates of olivine and pyroxene composition, crystalline olivines, crystalline pyroxenes, and crystalline silica - our goal is to find the best realistic opacities to represent these major kinds of dust, not necessarily to constrain the specific properties of the grains themselves (e.g., sizes, porosities, composition).

\section{2. $\quad$ Forsterite Opacity}

The first opacity for our models with which we concern ourselves is that of forsterite. Olivines have been used before in modeling dust emission to fit emission (and sometimes absorption) complexes at 10.0, 11.2, 16, 19, 23, 28, and $33 \mu \mathrm{m}$ (e.g., Molster et al. 2002). One spectrum for which these features appear at very high signal-to-noise ratio $(\mathrm{S} / \mathrm{N})$ is that 
of HBC 656, which we include as our first and most important forsterite exemplar. Others with these features in their spectra at fairly high $\mathrm{S} / \mathrm{N}$ ratios that we include as forsterite exemplars are DK Tau, GN Tau, IS Tau, ROXs 42C (which has already been identified as a silica exemplar; see Sargent et al. 2008), V836 Tau, and V955 Tau.

To fit the forsterite features in these exemplars' spectra, we have compiled opacities of crystalline olivine from a wide range of sources. From Jaeger et al. (1998), we include opacities from transmission measurements of the following olivine powders pressed into potassium bromide $(\mathrm{KBr})$ pellets: forsterite $\left(\mathrm{Mg}_{2} \mathrm{SiO}_{4}\right)$, magnesium-rich olivine $\left(\mathrm{Mg}_{1.8} \mathrm{Fe}_{0.2} \mathrm{SiO}_{4}\right)$, hortonolite $\left(\mathrm{MgFeSiO}_{4}\right)$, and fayalite $\left(\mathrm{Fe}_{2} \mathrm{SiO}_{4}\right)$. To explore further the effects on opacity of the full range of iron content in the olivine solid series (olivines of all $\mathrm{Mg} / \mathrm{Fe}$ ratios), we include 8 opacities measured by Koike et al. (2003) for the range of $0.77<\mathrm{x}<1.0$ for olivines of composition $\mathrm{Mg}_{2 x} \mathrm{Fe}_{2-2 x} \mathrm{SiO}_{4}$, and also $\mathrm{x}=0.218$. In addition, we created a hybrid forsterite opacity using the $\mathrm{x}=1$ ("Fo100") opacity from Koike et al. (2003) for $\lambda<15 \mu \mathrm{m}$ and roomtemperature forsterite opacity measured by Chihara et al. (2001) for $\lambda>15 \mu \mathrm{m}$. We also include the opacities of forsterite powder measured by Fabian et al. (2001) and annealed forsterite by Fabian et al. (2000). Finally, we compute opacities for the Continuous Distribution of Ellipsoids (see Bohren \& Huffman 1983) and CDE2 (see Fabian et al. 2001). CDE2 and CDE are distributions of ellipsoids whose size can be described as being in the "Rayleigh-limit", or characteristic size (of the semimajor axes of the ellipsoid) much less than $\frac{\lambda}{2 \pi}$, with ellipsoids described by every possible set of axial ratios weighted more toward near spherical shapes (CDE2) and equally (CDE), respectively. For both shape distributions of forsterite, we compute opacities using optical constants for the three crystallographic axes of forsterite provided by Sogawa et al. (2006). To account for this anisotropy of forsterite, we compute opacities for forsterite grains in a given shape distribution (CDE2 or CDE) three times, once for each of the 3 crystallographic axes' optical constants; we average these three resulting opacities to account for forsterite grains oriented randomly (see Bohren \& Huffman $1983)$.

As a starting mixture (not the same as the standard mixture determined and used later) of opacities for other components to use in modeling our forsterite exemplars, we use the non-forsterite opacities from Sargent et al. (2008); that is, CDE2 opacities for amorphous olivine and amorphous pyroxene using optical constants from Dorschner et al. (1995) for $\mathrm{MgFeSiO}_{4}$ and $\mathrm{Mg}_{0.7} \mathrm{Fe}_{0.3} \mathrm{SiO}_{3}$, respectively, "En90" enstatite opacity $\left(\mathrm{Mg}_{0.9} \mathrm{Fe}_{0.1} \mathrm{SiO}_{3}\right)$ from Chihara et al. (2002), and annealed silica opacity from Fabian et al. (2000). For large grains, we use the aforementioned amorphous silicate optical properties and Mie Theory (Bohren \& Huffman 1983) to compute the opacity of $5 \mu \mathrm{m}$ radius spheres that are $60 \%$ vacuum by volume, computing effective complex dielectric functions for this porous amorphous silicate material using Bruggeman Effective Medium Theory (EMT; Bohren \& Huffman 
1983).

The scattering efficiencies of small amorphous and crystalline silicate grains are negligible because they are in the Rayleigh limit for Spitzer IRS wavelengths and are not included in their opacities. The scattering component of opacity is not negligible for $5 \mu \mathrm{m}$ radius grains. The scattering cross section is comparable to the absorption cross section in the 10 $\mu \mathrm{m}$ region for both amorphous pyroxene and olivine dust grains. Since we model the emission from an optically thin region (the upper disk layers), only the absorption component contributes to our model. Full radiative transfer treatments of the emergent intensity from these optically thick disks must include the effects of scattering on the 5-37 $\mu \mathrm{m}$ IRS spectra. We note the detailed radiative transfer models of D'Alessio et al. (2001), who cite earlier work by Miyake \& Nakagawa (1993), show that scattering by large grains does not much change the flux from optically thin regions and makes the flux from optically thick regions slightly smaller. For this reason, we deem the lack of inclusion of scattering in opacities not of great concern.

We give in Table 1 the mass weights in units of our conservative uncertainties, $\tilde{a}_{k}$ (a measure of significance), of the olivine components for each of the seven forsterite exemplars. Those olivine opacities whose $\tilde{a}_{k}$ are highest contribute most significantly to the fit of a given forsterite exemplar. A given entry in a column in Table 1 is the mass weight of the component named in the first entry on the same row divided by its uncertainty, giving the significance of a particular opacity used in a given model. A prefix of "C" (or "cool") means the opacity was used at the lower temperature, and a prefix of "W" (or "warm") means the opacity was used at the higher temperature. Of the entries whose names include "J98" (meaning those opacities were presented by Jaeger et al. (1998)), "fo" denotes forsterite, "ol" denotes Mgrich olivine, "ho" denotes hortonolite, and "fa" denotes fayalite. The subsequent 9 opacities come from Koike et al. (2003), with the number after "Fo" indicating the stoichiometric abundance, expressed as a percentage, of $\mathrm{Mg}$ with respect to $\mathrm{Fe}$ in the ratio $\mathrm{Mg} /(\mathrm{Fe}+\mathrm{Mg})$ for olivine $[\mathrm{Mg}, \mathrm{Fe}]_{2} \mathrm{SiO}_{4}$. Entries for Fo100, Fo90.7, and Fo77, and the opacity of forsterite powder presented by Fabian et al. (2001) are not included at either temperature because no mass of any of them was ever used in any of the models of the forsterite exemplars. "annfor" denotes the opacity of forsterite presented by Fabian et al. (2000) annealed for the longest duration. "fohyb" is the hybrid opacity (explained in the text). S6 means the optical constants come from Sogawa et al. (2006), and CDE and CDE2 are the shape distributions for which opacities are computed using these optical constants. We find that a few opacities recur from model to model, and some of those at both model dust temperatures. Arrows in the final column indicate recurring opacities. Forsterite powder measured by Jaeger et al. (1998) shows up in 4 models as cool dust, but not as warm dust. Our forsterite "hybrid" opacity shows up in 3 models as cool dust, but only once as warm dust. Annealed forsterite 
occurs in 4 models as a warm dust component, but not as a cool component. Fayalite from Jaeger et al. (1998) occurs as warm dust with frequently high $\tilde{a}_{k}$ for the 6 exemplars, but as cool dust only once. The opacity that most repeatedly occurs as both warm and cool dust components in the models of the exemplars is the CDE2 opacity using optical constants for forsterite given by Sogawa et al. (2006). Frequently associated with this is also the CDE opacity for forsterite from the same optical constants.

The best fits seem to come from a combination of CDE2 and CDE shape distributions of forsterite using optical constants from Sogawa et al. (2006), suggesting that a shape distribution intermediate in weighting between CDE2 and CDE is indicated. Further, we note that the CDE shape distribution is somewhat unphysical, requiring ellipsoids either infinitely long or infinitesimally thin. We therefore computed an opacity curve for a custom-designed shape distribution of ellipsoids intermediate in weighting between that of CDE2 and of CDE. We note that a similar attempt was made by Zubko et al. (1996) resulting in what those authors called "modified Continuous Distribution of Ellipsoids". As the features for forsterite grains in the CDE shape distribution peak at wavelengths slightly longward of the peaks in the data and those of the CDE2 shape distribution of forsterite grains peak at wavelengths shortward of the peaks in the data, we expect the intermediate shape distribution to give opacity peaks at more optimal wavelengths than do the CDE or CDE2 shape distributions. Not knowing exactly how to weight one ellipsoidal shape relative to another, like CDE we weight all allowed shapes equally, but unlike CDE we do not allow ellipsoidal shapes with $\mathrm{L}_{j}$ parameter (see Bohren \& Huffman 1983) equal to zero in the shape distribution 2 . Our restricted shape distribution, which we call the "truncated Continuous Distribution of Ellipsoids", or tCDE, weighs all points equally within the bounds of a right triangle on the graph bounded by the three vertices $\left(\mathrm{L}_{1}, \mathrm{~L}_{2}\right)$ of $(0.005, \mathrm{t}),(0.005,0.99)$, and $(0.995-\mathrm{t}, \mathrm{t})$. We chose not to search for the best forsterite opacity using the tCDE shape distribution of forsterite and an untested enstatite opacity (En90), but, instead, to search jointly for the best value of the bound, t, on the tCDE shape distribution and the best enstatite opacity. This is because enstatite opacities have features of similar widths and at many of the same wavelengths $(10,19,23,28,33)$ as the opacities of forsterite.

\footnotetext{
${ }^{2}$ The $\mathrm{L}_{j}$ parameters are inversely proportional to the semimajor axis of one of the three ellipsoidal axes, there is one $\mathrm{L}_{j}$ parameter for each of the three axes, and the sum of the $\mathrm{L}_{j}$ parameters equals 1 . Therefore, two $\mathrm{L}_{j}$ parameters, $\mathrm{L}_{1}$ and $\mathrm{L}_{2}$, suffice to describe completely the shape of the ellipsoid. This can be represented graphically with $\mathrm{L}_{1}$ as the horizontal axis and $\mathrm{L}_{2}$ as the vertical axis. Any conceivable ellipsoidal shape is then represented on this graph as a point in the first quadrant satisfying $\mathrm{L}_{2}<1-\mathrm{L}_{1}$
} 


\subsection{Enstatite Opacity}

Now we focus on choosing the best opacity of enstatite, also searching for the best bound in the tCDE shape distribution for forsterite. Sargent et al. (2006) found FN Tau to require enstatite to fit narrow features in its $10 \mu \mathrm{m}$ complex at 9.3, 10.6, 11.2, and $11.6 \mu \mathrm{m}$. In this study, we are interested in modeling all silicate dust features found in IRS spectra, so we look for guidance longward of the $10 \mu \mathrm{m}$ complex to the spectrum of FN Tau. FN Tau has a very weak $16 \mu \mathrm{m}$ feature (unlike the forsterite exemplars), a strong $28 \mu \mathrm{m}$ feature relative to both the 23 and $33 \mu \mathrm{m}$ complexes, and a double-peaked $33 \mu \mathrm{m}$ complex. We look for these patterns elsewhere in our Taurus-Auriga sample to find other enstatite exemplars. DH Tau also has a narrow $9.3 \mu \mathrm{m}$ feature, strong $28 \mu \mathrm{m}$ complex, and double-peaked $33 \mu \mathrm{m}$ complex. We also include Haro 6-37 and HK Tau as enstatite exemplars because both have narrow features at 9.3, 10.6, and also between 11 and $12 \mu \mathrm{m}$, though they lack prominent crystalline silicate features at wavelengths longward of the $10 \mu \mathrm{m}$ complex.

As with the olivines, numerous lab measurements of pyroxenes exist. We include opacities computed from transmission spectra of submicron pyroxene powders in potassium bromide $(\mathrm{KBr})$ pellets for orthoenstatite $\left(\mathrm{MgSiO}_{3}\right.$ crystals in orthorhombic crystal structure), clinoenstatite $\left(\mathrm{MgSiO}_{3}\right.$ in monoclinic crystal structure), the aforementioned En90, "En80" $\left(\mathrm{Mg}_{0.8} \mathrm{Fe}_{0.2} \mathrm{SiO}_{3}\right)$, "En70" $\left(\mathrm{Mg}_{0.7} \mathrm{Fe}_{0.3} \mathrm{SiO}_{3}\right)$, "En60" ( $\left.\mathrm{Mg}_{0.6} \mathrm{Fe}_{0.4} \mathrm{SiO}_{3}\right)$, "En50" $\left(\mathrm{Mg}_{0.5} \mathrm{Fe}_{0.5} \mathrm{SiO}_{3}\right)$, and "En00" $\left(\mathrm{FeSiO}_{3}\right.$, or ferrosilite) by Chihara et al. (2002). We also consider similar measurements of synthetic orthoenstatite and clinoenstatite (Koike et al. 2000); natural clinoenstatite from Akita, Japan, orthoenstatite from Norway, and synthetic clinoenstatite and orthoenstatite (Koike et al. 2000); and hypersthene $\left(\mathrm{Mg}_{0.5} \mathrm{Fe}_{0.5} \mathrm{SiO}_{3}\right)$, bronzite $\left(\mathrm{Mg}_{0.8} \mathrm{Fe}_{0.2} \mathrm{SiO}_{3}\right)$, and clinoenstatite (Jaeger et al. 1998). We also compute opacities of orthoenstatite grains in the CDE2 shape distribution using optical constants for the three crystallographic axes provided by Jaeger et al. (1998). At the same time, for the rest of the dust types in the models, we use the same non-forsterite and non-enstatite opacities as were used in the models of the forsterite exemplars in the previous section, and we explore values of the bound, $t$, on the tCDE shape distribution of forsterite ranging from 0.04 to 0.1 . We also include the opacity of the CDE2 shape distribution of forsterite grains. We use all of these opacities to model the spectra of the enstatite exemplars FN Tau, DH Tau, Haro 6-37, and HK Tau, in addition to the forsterite exemplars.

Table 2 gives the significance of the mass weights of the opacities of crystalline pyroxene and crystalline olivine in the models of the enstatite and forsterite exemplars. The opacities named "en90", "en80", "en70", "en50", and "en00" were presented by Chihara et al. (2002). The ones named "J98CDE2" use the optical constants for the three crystallographic axes

of orthoenstatite presented by Jaeger et al. (1998) to compute opacity for grains in a CDE2 
shape distribution. "K0so" is the synthetic orthoenstatite, whose opacity is presented by Koike et al. (2000). "J98b" and "J98c" are bronzite and clinoenstatite opacities, respectively, presented by Jaeger et al. (1998) obtained via transmission measurements of crushed grains pressed into pellets. Entries for orthoenstatite, clinoenstatite, and "en60" opacities from Chihara et al. (2002), synthetic clinoenstatite from Koike et al. (2000), and hypersthene from Jaeger et al. (1998) are not included at either temperature because no mass of any of them was ever used in any of the models of the exemplars of both forsterite and enstatite modeled jointly. "S6" in the last five rows refers to optical constants presented by Sogawa et al. (2006) used to compute opacities for the CDE2 shape distribution (the first of the five) and for the tCDE shape distributions with bounding parameter (see discussion in Section 3.4), t, of $0.10,0.08,0.06$, and 0.04 for the second through fifth of the five opacities.

Among the pyroxene opacities, CDE2 orthoenstatite from optical constants provided by Jaeger et al. (1998) shows up at significant levels as a cool dust component but only weakly as a warm dust component. Ferrosilite (En00) by Chihara et al. (2002) often contributes significantly as a warm dust component but not as a cool component. The one opacity that contributes significantly most frequently as both a warm and a cool dust component is the clinoenstatite (enstatite having monoclinic crystalline structure) En90 from Chihara et al. (2002). Though we use the opacity of enstatite of composition $\mathrm{Mg}_{0.9} \mathrm{Fe}_{0.1} \mathrm{SiO}_{3}$ having slight iron content, the fit at long wavelengths is not ideal and suggests caution in the interpretation of finding crystalline silicates with any iron content. This opacity was used by Sargent et al. (2006) in modeling the $10 \mu \mathrm{m}$ complexes of 12 TTS and by Sargent et al. (2008) in modeling silica exemplars. Among the various opacities of forsterite, the tCDE opacity with bound $\mathrm{t}=0.1$ gives the best fit to both the enstatite and forsterite exemplars. We note the improve-

ment of fit of the spectrum of ROXs 42C reported by Sargent et al. (2008) when using tCDE instead of CDE forsterite (optical constants by Sogawa et al. 2006), with $\chi^{2}$ per degree of freedom of 4.3 and 5.1, respectively.

\subsection{Amorphous Silicate Opacities}

Now we turn to amorphous silicate opacities. Sargent et al. (2006) showed that the transitional disks (disks whose outer parts are optically thick to mid-infrared radiation but whose inner regions are very optically thin) CoKu Tau/4, DM Tau, and GM Aur were fit well using opacities of mostly submicron amorphous silicates having smooth 10 and $20 \mu \mathrm{m}$ features. LkCa 15 was shown by Espaillat et al. (2007) to be a pre-transitional disk, which are like the aforementioned transitional disks but with more dust in the inner regions. As with the transitional disks, LkCa 15 shows smooth 10 and $20 \mu \mathrm{m}$ features. FM Tau, TW 
Cha, and UY Aur also show similarly smooth 10 and $20 \mu \mathrm{m}$ features. We consider these seven objects to be amorphous silicate exemplars.

Dorschner et al. (1995) gives complex indices of refraction for amorphous dust of pyroxene composition ("amorphous pyroxene") $\mathrm{MgSiO}_{3}, \mathrm{Mg}_{0.7} \mathrm{Fe}_{0.3} \mathrm{SiO}_{3}$, and $\mathrm{Mg}_{0.5} \mathrm{Fe}_{0.5} \mathrm{SiO}_{3}$ and also for amorphous dust of olivine composition ("amorphous olivine") $\mathrm{MgFeSiO}_{4}$. We use complex indices of refraction for samples of amorphous forsterite $\left(\mathrm{Mg}_{2} \mathrm{SiO}_{4}\right)$ and amorphous enstatite $\left(\mathrm{MgSiO}_{3}\right)$ obtained by laser ablation of crystals of forsterite and enstatite, respectively (Scott \& Duley 1996). We also use complex indices of refraction for samples of amorphous forsterite and amorphous enstatite prepared by sputtering of samples of MgSi and $\mathrm{Mg}_{2} \mathrm{Si}$ in an atmosphere of 50:50 argon-oxygen Dav (1979). Additionally, we use optical constants for a sample of amorphous bronzite obtained by quenching a melt of a natural sample of bronzite $\left(\mathrm{Mg}_{0.9} \mathrm{Fe}_{0.1} \mathrm{SiO}_{3}\right)$ from Paterlestein, Germany analyzed by Dorschner et al. (1988) and for amorphous pyroxene of cosmic composition $\left(\mathrm{Ca}_{0.03} \mathrm{Mg}_{0.52} \mathrm{Fe}_{0.45} \mathrm{SiO}_{3}\right.$; sample "1S") by Jaeger et al. (1994). For the same reasons provided by Sargent et al. (2006), we assume the amorphous silicate dust grains are in the CDE2 shape distribution.

We also compute opacities of $5 \mu \mathrm{m}$ radius, $60 \%$ vacuum porous grains. The narrow features in the opacity curves of crystalline grains are very sensitive to the exact details of these grains' properties such as shape, composition, porosity, size, etc, as we have already indicated in our exploration of the opacities of submicron crystalline grains used to fit silica, forsterite, and enstatite exemplars. For this reason, we, as Bouwman et al. (2001), do not include opacities of large crystalline grains. The opacities for large amorphous silicate grains are less sensitive to these details.

Large grains (greater than $1 \mu \mathrm{m}$ in characteristic size) in protoplanetary disks should not be homogeneous. Instead, they should be like Interplanetary Dust Particles, which are heterogeneous aggregates whose components are $\sim 0.1 \mu \mathrm{m}$ in size (see Harker et al. 2002) and composed chiefly of either amorphous silicate, forsterite, or enstatite (see the review by Bradley 2003). How should the opacities of such large heterogeneous grains appear? The recent study by Min et al. (2008) concluded that the opacity of a large heterogeneous aggregate is equal to the sum of opacities of its constituents, the opacities resembling those of homogeneous grains of characteristic size corresponding to the abundance of the particular constituent in the large aggregate. As an example in their study, they compute the opacity of a large porous aggregate of amorphous silicate, forsterite, and enstatite, the amorphous silicate being the most abundant component of the aggregate. The opacity of the amorphous component of the aggregate resembled that of homogeneous grains of amorphous silicate of size similar to that of the heterogeneous aggregate, while for the lowest abundance of crystalline grains in the aggregate, the forsterite contribution to the aggregate opacity resembled 
the opacity of much smaller grains of homogeneous forsterite. Very similar conclusions were also reached by Bouwman et al. (2008), finding that the typical grain size of crystalline silicate grains used in their models of seven spectra were submicron, but typical amorphous silicate grain sizes were up to $6 \mu \mathrm{m}$ radius (solid grains).

Because the grains in protoplanetary disks grow primarily by sticking together, it is likely large grains will be heterogeneous, composed of various sub-micron components, similar to the IDPs. The small amorphous components are believed to come directly from the ISM, with sizes $<0.25 \mu \mathrm{m}$ in radius. Since nearly all our objects show evidence for this amorphous component, one expects a significant amorphous component in the large, porous grains. Furthermore, the amorphous components were present at the initial formative stages of the protoplanetary disks. On the other hand, the crystalline components were produced later, by as yet unknown processes in the protoplanetary disks (e.g., van Boekel et al. 2004; Sargent et al. 2006; Watson et al. 2008; Bouwman et al. 2008). Thus one would expect amorphous components to dominate the heterogeneous fluffy grains. In this case, the large grain spectra will resemble the sum of completely amorphous large grains with an admixture of sub-micron crystalline grains. The models presented here will correctly detect the large grains, through the broadening of the silicate features to longer wavelengths. Any crystalline components of large grains will be modeled as small crystalline grains. The opacity of a large porous heterogeneous grain with a significant abundance of crystalline components, however, will not be modeled well by our standard set of opacities, especially at longer wavelengths $(\lambda>20 \mu \mathrm{m})$ in the IRS spectra (see Appendix B). Because of optical depth effects, this will somewhat underestimate the crystalline mass fraction contributed by large grains.

To model our amorphous silicate exemplars, we use annealed silica, enstatite, and forsterite in the tCDE shape distribution (with bound $t=0.1$ ) as our crystalline silicate opacities. We use the aforementioned amorphous silicate optical properties for grains in the CDE2 shape distribution to compute opacities of submicron grains of amorphous pyroxene and amorphous olivine and also of $5 \mu \mathrm{m}$ radius, $60 \%$ vacuum grains of amorphous pyroxene and amorphous olivine. In testing the amorphous pyroxene and amorphous olivine opacities, we chose the material whose opacities of both submicron and $5 \mu \mathrm{m}$ grains showed up most frequently as a significant contributor as both warm and cool dust. In Table 3, we list the significance of the mass weights of the amorphous pyroxenes and amorphous olivines used in the models of our seven amorphous silicate exemplars. "D95" refers to optical constants presented by Dorschner et al. (1995), "D79" refers to optical constants presented by Dav (1979), "SD96" refers to optical constants presented by Scott \& Duley (1996), and "J94" refers to optical constants presented by Jaeger et al. (1994). The amorphous olivine "Ol" from Dorschner et al. (1995) is $\mathrm{MgFeSiO}_{4}$, and the amorphous olivine from Dav (1979) is amorphous forsterite. The amorphous pyroxenes with "Py5" and "Py10" in their names 
refer, respectively, to $\mathrm{Mg}_{0.5} \mathrm{Fe}_{0.5} \mathrm{SiO}_{3}$ and $\mathrm{MgSiO}_{3}$ presented by Dorschner et al. (1995). The amorphous pyroxene from Jaeger et al. (1994) is amorphous pyroxene of cosmic composition, and the amorphous pyroxenes from Day (1979) and Scott \& Duley (1996) are amorphous enstatite. "Sm" denotes small Rayleigh-limit (here, submicron) size grains in the CDE2 shape distribution. "Lg" denotes large $5 \mu \mathrm{m}$ radius $60 \%$ vacuum porous spheres. Entries for opacities computed from optical constants for amorphous forsterite from Scott \& Duley (1996), amorphous pyroxene of composition $\mathrm{Mg}_{0.7} \mathrm{Fe}_{0.3} \mathrm{SiO}_{3}$ from Dorschner et al. (1995), and amorphous bronzite from Dorschner et al. (1988) are not included at either temperature because no mass of any of them was ever used in any of the models of the amorphous silicate exemplars.

Although it is never used as a cool dust component, the amorphous olivine $\mathrm{MgFeSiO}_{4}$ from Dorschner et al. (1995) is the best amorphous olivine because it is a very significant warm dust component in the models of all seven amorphous exemplars. LkCa 15 provides one of the most stringent tests of the amorphous silicate features, as its silicate features are very prominent and have high S/N ratios. Amorphous olivine (Dorschner et al. 1995) almost exclusively fits this spectrum's $10 \mu \mathrm{m}$ feature. Amorphous pyroxene can dominate as a cool dust component over amorphous olivine; for instance, the amorphous pyroxene of cosmic composition (Jaeger et al. 1994) exclusively fits the $20 \mu \mathrm{m}$ feature of LkCa 15. Although the rest of the amorphous pyroxenes are fairly equal contenders for best amorphous pyroxene opacity, the amorphous pyroxene of cosmic composition by Jaeger et al. (1994) shows up as the most significant amorphous pyroxene opacity in the most exemplars, so we choose this as our amorphous pyroxene opacity. The minor modification to the amorphous pyroxene opacity usually improved (but sometimes made worse) the $\chi_{\nu}^{2}$ by $\sim 0.2$ over the amorphous pyroxene opacity used by Sargent et al. (2006, 2008).

The amorphous pyroxene of cosmic composition used in our models has a magnesium-toiron ratio of 52:45, while the amorphous olivine used has a Mg-to-Fe ratio of 50:50. These are not far from the Mg-to-Fe ratio for the "cosmic" abundances of these two elements (Mg:Fe $\sim$ 4:5, measured by Holweger et al. 1990; Snow \& Witt 1995; Cox 2000). We also note the imaginary parts of the complex dielectric functions in the visible and near-infrared regions (0.2-8 $\mu \mathrm{m}$ wavelengths) of the best amorphous olivine (Dorschner et al. 1995) and the best amorphous pyroxene (Jaeger et al. 1994) are very similar to that of "astronomical silicate" (Draine \& Lee 1984), whose near-ultraviolet, visible, and near-infrared imaginary part of the dielectric function was specifically constructed so that astronomical silicate grains would heat to the correct temperatures in the presence of stars. This was important in attempting to obtain a self-consistent model of the Spitzer IRS spectrum of IP Tau by Sargent et al. (2006). We use the best opacities that fit our various forsterite, enstatite, and amorphous silicate exemplars to fit our sample of 65 spectra of TTS from the Taurus-Auriga star-forming 
region.

The first real test of this ensemble of dust opacities to be used as a standard mixture for dust emission models was to fit the mid-infrared spectrum of the Trapezium nebulosity. The 8-13 $\mu \mathrm{m}$ spectrum of the Trapezium (Forrest et al. 1975) was used by Draine \& Lee (1984) to derive the emissivity of "astronomical silicate" grains, which represent the dust in the Interstellar Medium. The 8-13 $\mu \mathrm{m}$ spectrum of the Trapezium presented by Forrest et al. (1975) was combined with the $16-38 \mu \mathrm{m}$ spectrum of the Trapezium obtained by subtracting $3 \%$ of the spectrum of the Kleinman-Low nebula from the spectrum obtained pointed at the Trapezium (see Forrest et al. 1976). This accounted for contamination by emission from the KL nebula of the wide beam of the detector while pointed toward the Trapezium (Forrest et al. 1976). The best-fit model for the Trapezium spectrum is shown in Figure 1.

As can be seen, the dominant components used to fit the spectrum are submicron amorphous pyroxene and submicron amorphous olivine. This is consistent with more recent analyses of dust composition of the ISM along the line-of-sight to the Galactic Center by Kemper et al. (2004), who found their dust models required negligible large grains and very small amounts of crystalline silicates (less than $2.2 \%$ by mass). The parameters for the model shown in Figure 1 are given in Table 4.

We describe in the Appendix our tests of Bruggeman effective medium theory used to compute effective complex dielectric functions and Mie Theory used to compute opacities of large grains.

\subsection{Degeneracy of Model Components}

We desire to measure the degeneracy between model components. This is the extent to which one component could be replaced by other components and achieve a similarly good model fit. After computing the best-fit models (to be described in the next chapter) for the spectra of FN Tau (an enstatite exemplar), IS Tau (a forsterite exemplar), ZZ Tau (a silica exemplar), and DM Tau (an amorphous silicate exemplar), $\chi^{2}$ was minimized with respect to the blackbody solid angles and dust mass weights over 7.7-37 $\mu \mathrm{m}$ wavelengths at the two dust temperatures (found by the best-fit models of these exemplars), not eliminating components with negative solid angle or mass weight. In the process of minimizing $\chi^{2}$, a $16 \times 16$ element covariance matrix was computed, each of the rows and columns of which belong to one of the 7 dust species or the blackbody at one of two temperatures. Each of the elements of this matrix is a sum over all concerned wavelengths $(7.7-37 \mu \mathrm{m})$ of a product. The product is opacity times the Planck function (or just the Planck function in the case of 
a blackbody component) specific for a given row times the opacity times the Planck function (or, again, just the Planck function in the case of a blackbody component) specific for a given column, all divided by the square of the flux density uncertainty. The inverse of this matrix gives the covariance matrix (see discussion in Chapter 14 of Press et al. 1992). The diagonal elements of this matrix are the variances of each of the dust components in the model, and the off-diagonal elements are the covariances of the dust component of a given row with the dust component of a given column.

The correlation coefficient, $r$, is computed for each off-diagonal element by dividing the covariance of that element by the square root of the product of the variances of the two dust components in question. One variance is the diagonal element of the same column, the other is that of the same row. Two highly degenerate components will be highly anticorrelated, with a correlation coefficient very near -1 . A correlation coefficient near zero means the two components are not correlated. In addition, for each off-diagonal element, the probability, P, of a coefficient of equal or greater magnitude being found for a non-correlated data set (which is the probability of the correlation coefficient having been drawn from a random distribution; see Taylor 1982) is computed. P near 0\% indicate significant correlation coefficients, and P near $100 \%$ indicate insignificant correlation coefficients.

Here we discuss the most significantly degenerate component pairs, which are shown in Table 5. The most negative r's are for the pair of cool large amorphous olivine and cool large amorphous pyroxene. For this pair, $\mathrm{r}$ is between -0.87 and -0.89 (and $\mathrm{P}=0.0 \%$ ) for the four representative exemplars FN Tau (enstatite exemplar), IS Tau (forsterite exemplar), ZZ Tau (silica exemplar), and DM Tau (amorphous silicate exemplar), indicating the most degeneracy between components. Warm large amorphous olivine and warm large amorphous pyroxene are also fairly highly anticorrelated (degenerate); for this pair, $\mathrm{r}$ is usually around 0.72 over the sample of 65 spectra. Similarly, cool small amorphous pyroxene and cool small amorphous olivine are fairly significantly degenerate with each other, with $-0.82>\mathrm{r}>-0.89$ over the four representative exemplars $(\mathrm{P}=0.0 \%)$. The warm small amorphous pyroxene and amorphous olivine are also highly degenerate, with $\mathrm{r}$ usually near -0.73 over the sample of 65 spectra. Cool enstatite is typically fairly significantly degenerate with cool forsterite, with $\mathrm{r}$ usually near $\mathrm{r}=-0.46$ over the sample of 65 spectra, as enstatite and forsterite share very similar features in their opacities for wavelengths longward of the $10 \mu \mathrm{m}$ complex. Silica also shares a $20 \mu \mathrm{m}$ feature close to $19 \mu \mathrm{m}$ complex of enstatite, explaining the spread of $-0.29 \geq \mathrm{r} \geq-0.50(\mathrm{P} \leq 0.1 \%)$ in this pair of components for the four representative exemplars. At warm temperatures, enstatite is only significantly degenerate with silica, with $r$ for the 65 spectra in the sample usually being about -0.50, as warm silica and warm enstatite have strong, narrow features at 9.3 and $9.1 \mu \mathrm{m}$, respectively, while warm forsterite is not degenerate with either, not sharing a strong feature with silica or enstatite in the $10 \mu \mathrm{m}$ 
region.

For the highly degenerate pairs, the dust emission models accurately determine the sum of the masses of the two components, but not so accurately the individual masses. This indicates the model finding that the inner disk (warm component) tends to be dominated by amorphous olivine while the outer disk (cool component) shows more amorphous pyroxene is probably not physically real. This, at least partially, explains the negative masses often found to give the very best fit. By zeroing the negative mass, the complementary component's mass is increased to give nearly as good a fit. Figure 2 demonstrates this effect by showing the degeneracy between cool small amorphous pyroxene and cool small amorphous olivine for the model of the Trapezium shown in Figure 1. For the best-fit model temperature pair, the model is recomputed but without setting any of the components in the model to zero, allowing mass weights to be negative if that results in the lowest $\chi^{2}$ per degree of freedom (reduced $\chi^{2}$ ). All resulting model parameters are held at their new best-fit values except for the mass weights of cool small amorphous pyroxene and cool small amorphous olivine, which are varied over the ranges indicated on the plot. The levels of reduced $\chi^{2}$ resulting from exploring this range of mass weights of these two dust components are shown as contours on the plot, with the levels of the first three contours indicated. This contour plot suggests that replacing some amount of one of the cool small amorphous silicates with the same mass of the other gives a very similarly good fit, changing reduced $\chi^{2}$ only very slightly.

Histograms of the correlation coefficient between four representative pairs of dust components for all 65 spectra in the sample are given in Figure 3. The histograms all show single, well-defined peaks. This indicates the correlation coefficients are measuring real degeneracy between model components.

\section{Results}

Dust emission models are fit to the 65 Taurus-Auriga spectra, which we show in Figures 4-14. Table 6 gives the parameters of these models (temperatures, blackbody solid angles, mass weights, reduced $\left.\chi^{2}\right) \cdot \chi^{2}$ is minimized over 7.7-37 $\mu \mathrm{m}$, so both dust temperatures used in the models are well constrained by relative uncertainties of $10 \%$. The median high and low model dust temperatures are $545 \mathrm{~K}$ and $127 \mathrm{~K}$, respectively. In the DR Tau model, which has dust at precisely these temperatures, most of the $10.0 \mu \mathrm{m}$ wavelength flux above that from the blackbodies originates from $545 \mathrm{~K}$ dust, while most of the $20.0 \mu \mathrm{m}$ flux above that from the blackbodies originates from $127 \mathrm{~K}$ dust. Assuming a distance of $140 \mathrm{pc}$ to DR Tau, assuming all of the optically thin dust flux at $10.0 \mu \mathrm{m}$ comes from $545 \mathrm{~K}$ dust in a disk of total optical depth at $10.0 \mu \mathrm{m}$ of 0.1 , and assuming all of the optically thin dust flux at 
$20.0 \mu \mathrm{m}$ comes from $127 \mathrm{~K}$ dust in a disk of total optical depth at $20.0 \mu \mathrm{m}$ of 0.1 , the radii of these two disks in the case of DR Tau are 0.75 AU and 11.5 AU for the warm and cool optically thin dust, respectively. We list at the end of Table 6 the best opacities that fit our various forsterite, enstatite, and amorphous silicate exemplars to fit our sample of 65 spectra of TTS from the Taurus-Auriga star-forming region.

\subsection{Extreme Inner Disk Grain Growth}

The spectra of DM Tau, DO Tau, UZ Tau/e, XZ Tau, and ZZ Tau IRS in our sample show evidence of high abundances of large grains. Figure 4 shows our models of these five spectra, breaking into components the model of UZ Tau/e as an example of the models. Warm large amorphous olivine dominates the $10 \mu \mathrm{m}$ complexes of these five objects' spectral models. The $20 \mu \mathrm{m}$ features also are partly fit by this same component, but usually more of the flux required to fit the $20 \mu \mathrm{m}$ features comes from cool submicron amorphous pyroxene. The overall shape of the spectrum of DM Tau differs from that of the other spectra in Figure 4 because it is a transitional disk (Calvet et al. 2005), lacking significant dust closer to the central star than $\sim 3$ AU. We note that these five spectra support our use of opacities of large grains of amorphous silicates and do not require those of large crystalline grains. That our models fit our spectra well is consistent with amorphous silicates being more abundant both as small grains and as constituents in larger grains (see Section 3.4). We note that significant abundances of large grains have been found for SR20, a TTS in the Ophiuchus star-forming region (McClure et al. 2008), CS Cha (Espaillat et al. 2007), and CVSO 224 Espaillat et al. (2008). Furlan et al. (2007) found a large abundance of large grains around HD 98800 B, a somewhat older ( 10 Myr old) YSO in the TW Hydrae association. Large

abundances of large grains around other somewhat older stars were found by Bouwman et al. (2008) and Kessler-Silacci et al. (2006).

\subsection{Prominent Forsterite Spectral Features}

In Figure 5 we show the spectra of six spectra with prominent forsterite features, five of which are our forsterite exemplars, with the model of IS Tau broken down into components. We note in support of the forsterite opacities used in our models that the fits at all wavelengths to the spectra of these stars, DK Tau, F04147+2822, GN Tau, IS Tau, V836 Tau, and V955 Tau are quite good. All major features and complexes of forsterite at 10.0, 11.2, $16,19,23$, and $33 \mu \mathrm{m}$ are fairly well fit. 
The spectrum of F04147+2822 (Figure 5) is especially interesting. Its $10 \mu \mathrm{m}$ complex lacks any of the distinctive narrow emission features characteristic of the forsterite, enstatite, or silica and is fit well by a combination of emission from submicron amorphous silicate grains and large amorphous pyroxene. At longer wavelengths, however, emission features at 19, 23, and $33 \mu \mathrm{m}$ characteristic of forsterite dominate and, correspondingly, are fit well by our forsterite profile for grains in a tCDE shape distribution. This suggests a greater abundance of forsterite in the outer disk than the inner disk. One possible explanation is that this spectrum is a sum of emission from two disks, one with very little crystallinity throughout and blue in continuum color and another disk with a lot of crystallinity throughout the disk and red in continuum color, but this system is not known to be multiple. If this spectrum is from only one protoplanetary disk, this would contrast with the finding by van Boekel et al. (2004) of greater crystallinity within 2 AU than outside of 2 AU in disks around 3 Herbig Ae/Be stars. However, the two are not inconsistent, as T Tauri stars are less luminous than Herbig Ae/Be stars. Two AU in HAeBe stars should correspond to a much lesser radius in disks around $\mathrm{T}$ Tauri stars. It suggests that, on average, the regions to which we refer as "inner" and "outer" disk regions in the population of $65 \mathrm{~T}$ Tauri stars in Taurus-Auriga are both outside of the regions in T Tauri star disks analogous to the region inside $2 \mathrm{AU}$ of Herbig Ae/Be disks.

In some spectra, however, the long-wavelength side of the $10 \mu \mathrm{m}$ feature is not fit well. DD Tau, DE Tau, and V710 Tau all share this problem, as is seen in Figure 6, with the model of FX Tau broken into its components. There are many ways by which forsterite opacity features can be centered at longer wavelengths than those in our tCDE opacity curve. Grain shape distributions more heavily weighted toward extreme shapes (extremely flat, extremely elongated), larger grains (or large aggregates of small grains, with a large fraction of these small grains being forsterite), olivine grains with nonzero $\mathrm{Fe} / \mathrm{Mg}$ ratios, and forsterite grains with greater porosity would all result in forsterite emission features at longer wavelengths than resulted from the forsterite tCDE curve (solid spheres of forsterite give rise to opacity features at shorter wavelengths than those of forsterite in the CDE shape distribution, and the opacity curve of $60 \%$ porous spheres of forsterite is almost identical to that of forsterite in the CDE shape distribution Sargent et al. 2006). Also, both CoKu Tau/3 and FX Tau (Fig. 4) have 23 and $33 \mu \mathrm{m}$ emission complexes with emission that extends to longer wavelengths than provided by the submicron forsterite grains in the tCDE shape distribution in our models. That both spectra lack significant $28 \mu \mathrm{m}$ complexes indicates the dominant form of crystals giving rise to 20-37 $\mu \mathrm{m}$ emission for these two systems is forsterite and not enstatite. It should be noted, though, that the $10 \mu \mathrm{m}$ complexes in the spectra of both CoKu Tau/3 and FX Tau are fit fairly well. We therefore conclude there is variation in the exact details of the olivine grain populations in the protoplanetary disks in our sample, in terms of average 
grain shape, grain size, grain composition, and grain porosity. For DD Tau, DE Tau, and V710 Tau, this variation occurs for the warmer forsterite grains, while for CoKu Tau/3 and FX Tau, this variation occurs for the cooler forsterite grains. We note that most of these five also seem to have emission features at $14 \mu \mathrm{m}$ wavelength (see below, Section 4.8). Whatever gives rise to these $14 \mu \mathrm{m}$ features is not necessarily related to the poor fits to the forsterite features because the poor fitting happens over very limited wavelength ranges of $11-11.5 \mu \mathrm{m}$ and 23-25 $\mu \mathrm{m}$.

\subsection{Variation in Silica}

In addition to the olivine component of our models, the silica component may also vary in details of grain properties among all disks in our sample. While the silica features at 9, 12.6, and $20 \mu \mathrm{m}$ in the spectrum of ZZ Tau (and the other silica exemplars) are fairly fit well with the annealed silica opacity used, others like the $12.6 \mu \mathrm{m}$ features in the spectra of DN Tau and FZ Tau are not quite so well fit (see Figure 7). FZ Tau has a single-peaked feature in this wavelength range, but it peaks at $12.45 \mu \mathrm{m}$, shortward of the wavelength at which annealed silica peaks. This could suggest the polymorph of silica giving rise to this feature is $\alpha$-quartz at somewhat elevated temperatures of 500-625 K (see discussion by Sargent et al. 2008). DN Tau appears to have a double-peaked feature, which would suggest $\alpha$-quartz at $\sim 300 \mathrm{~K}$ (Sargent et al. 2008), but the S/N at these wavelengths is low for this spectrum so its reality is more doubtful (note the discussion on the effect of unresolved emission lines in this section). According to Sargent et al. (2008), the presence of silica argues for high-temperature processing, as it is not present in the interstellar medium and therefore should not be present as the starting dust mixture as the protoplanetary disk formed from its protostellar envelope. If $\alpha$-quartz can be confirmed, the silica, once formed, must cool slowly enough to allow phase transition from a higher-temperature polymorph, like tridymite or cristobalite, to $\beta$-quartz, then $\alpha$-quartz.

\subsection{Enstatite Exemplars}

We show the model fits to our enstatite exemplars in Figure 8, with the model of FN Tau broken into components. Other than a mismatch between model and spectrum for FN Tau at $11.6 \mu \mathrm{m}$, the fits to the $10 \mu \mathrm{m}$ complexes are generally good. However, we note the poorness of fit of our models to the longest wavelength complexes (28 and $33 \mu \mathrm{m})$ of the spectra of FN Tau and DH Tau, our two best enstatite exemplars. The height-to-continuum ratios in our models of these two exemplars for the 28 and $33 \mu \mathrm{m}$ complexes are unacceptably low 
compared to those in the spectra. We note the progressively increasing height-to-continuum ratio of the 19, 23, 28, and $33 \mu \mathrm{m}$ complexes in the spectra of both DH Tau and FN Tau. The rest of our sample are sufficiently fit using crystalline dust at two temperatures. This suggests that a population of submicron enstatite or forsterite dust at a third (very low) temperature would be required to give acceptable fits to these spectra. We note this lack of a third model temperature is not a problem for spectra requiring abundant forsterite (Figures 5 and 6). Note in Figure 6 the problem is not insufficient peak-to-continuum ratio for the model forsterite features; rather, the problem is insufficient width of the features, which is a problem with the forsterite opacity.

The LH part of the spectrum of Haro 6-37 (19.3-37 $\mu$ m wavelength) suffers from an artifact, in which each of the 10 spectral orders are tilted such that the flux density of their short-wavelength end is higher than it should be and the flux density of their long-wavelength end is lower than it should be. These tilted spectral orders, which could be interpreted to resemble narrow crystalline silicate features longward of $19.3 \mu \mathrm{m}$ wavelength, result from an artifact of the reduction of the LH spectrum of this object. This artifact could be due either to unsubtracted sky emission or to mispointing of the telescope with respect to Haro 6-37 along or perpendicular to the length of the LH slit.

\subsection{Amorphous Silicate Exemplars and Transitional Disks}

Spectra requiring abundant submicron amorphous silicate grains and model fits to these spectra are shown in Figures 9 and 10. Figure 9 shows the spectra and corresponding models of five of our amorphous silicate exemplars (see Section 3.4) and the model breakdown into components for FM Tau. In general, from 7.7 to slightly longward of $30 \mu \mathrm{m}$ wavelength, the fits of the models to the data are excellent. For GM Aur and especially LkCa 15, the each model fits the continuum at wavelengths greater than $30 \mu \mathrm{m}$ with emission from a weak $33 \mu \mathrm{m}$ complex of forsterite. This is an artifact, as these two spectra require a third model temperature to fit the data using emission from a blackbody or amorphous silicates at a very low temperature. Figure 10 shows spectra whose models also require relatively high abundances of submicron amorphous silicates, though they require more crystalline silicates and large grains than those shown in Figure 9; the model of HQ Tau is broken into its components as an example. Again, the model fits to the data are very good, with the exception of a slight insufficiency in the peak-to-continuum ratio of the forsterite $33 \mu \mathrm{m}$ complex in the model of DP Tau. 


\subsection{Small-Equivalent-Width $10 \mu \mathrm{m}$ Complexes}

The spectra of CY Tau, DF Tau, DG Tau, DL Tau, DQ Tau, IT Tau, and V807 Tau show $10 \mu \mathrm{m}$ complexes of small equivalent width (see Figure 11; the model of DF Tau is broken into components). CY Tau, DF Tau, DL Tau, and IT Tau also have essentially flat spectra over all IRS wavelengths, being consistent with the models presented by Furlan et al. (2006) and their interpretation of settling of dust grains. Settling of dust in a disk results in the disk being flatter and less flared, which gives rise to a bluer spectral continuum color in the mid-infrared and also less equivalent width in the distinctive silicate features. Andrews \& Williams (2005) noted CY Tau for its flat spectral colors over infrared wavelengths.

These spectra are well fit mostly by emission from submicron dust grains and the two blackbody components in the models. CY Tau requires a small amount of silica to fit in the $10 \mu \mathrm{m}$ complex and a moderate amount of cool forsterite to fit longer wavelength complexes, especially the $33 \mu \mathrm{m}$ feature. The emission required by large grains in the model for DF Tau to fit the longer wavelengths of its spectrum is the largest of the five low $10 \mu \mathrm{m}$ equivalent width spectra, in addition to small amounts of emission from submicron dust grains. Little emission from forsterite is required to give rise to the $10 \mu \mathrm{m}$ feature of DG Tau, while a fair amount of submicron amorphous pyroxene is required to fit its mild $20 \mu \mathrm{m}$ feature. The $10 \mu \mathrm{m}$ feature of DL Tau requires modest amounts of emission from forsterite and silica and lesser amounts from other kinds of dust. The LH spectrum (19.3-37 $\mu \mathrm{m})$ of DL Tau suffers from an order-tilt artifact as does Haro 6-37 (see above), limiting the precision with which we can determine the crystallinity and large grain content of the cooler outer disk, but it appears to be optimally fit using a mixture of both amorphous and crystalline grains. DQ Tau has subtle features at 9.3, 9.8, 10.6, 11.1, and $11.6 \mu \mathrm{m}$ that require a moderate amount of enstatite. IT Tau requires small amounts of many of the submicron amorphous and crystalline grains to fit its miniscule $10 \mu \mathrm{m}$ complex, but its broad $20 \mu \mathrm{m}$ feature is fit well by submicron amorphous pyroxene. The same applies to the fit of the $20 \mu \mathrm{m}$ feature of V807 Tau, though it requires a modest amount of large amorphous pyroxene to fit its 10 $\mu \mathrm{m}$ feature. The abundance of crystalline grains with respect to amorphous grains in the

models of these five spectra is consistent with the finding by Watson et al. (2008) using all (and more) of the spectra analyzed in this study that increased crystallinity accompanies more advanced settling of dust in disks as measured by continuum indices $n_{6-13}$ and $n_{13-31}$. It is also noted that these seven spectra with small equivalent width $10 \mu \mathrm{m}$ features do not indicate large abundances of large grains (see Table 6). We explore these issues more in Section 5 . 


\subsection{Mixed Dust Compositions}

In Figures 12-14, we show the spectra and corresponding models to the rest of our sample, with eight spectra in each figure arranged top-to-bottom alphabetically by their names. For all of these spectra, the abundances of the various dust species required by the models are mixed. Typically, no one dust type has an abundance required by its model much more than the abundances of any of the other dust types in that model. The spectrum of AA Tau (and others) have what appear to be a broad emission feature at $14 \mu \mathrm{m}$, but this probably originates from gas emission (Carr \& Najita 2008); also, SH and LH spectra of AA Tau and a few other objects show unresolved emission lines from $\mathrm{H}_{2} \mathrm{O}$ and $\mathrm{OH}$ (see next subsection). The models of FP Tau, GI Tau, and VY Tau are broken into components in Figures 12, 13, and 14, respectively. Note that FS Tau and FV Tau in Figure 13 and GK Tau, HN Tau, and Haro 6-28 in Figure 14 suffer from the same LH order-tilt artifact as did Haro 6-37 (see Section 4.4). Also note the high point-to-point noise in the 20-25 $\mu \mathrm{m}$ parts of the LL spectra of FT Tau, GG Tau B, and GH Tau in Figure 13 and IQ Tau in Figure 14. This "fringing" artifact is due to the delamination of the LL order-sorting filter and shows up when the telescope has been mispointed with respect to a source in the direction perpendicular to the slit for a LL observation, with increasing severity of the artifact for greater mispointing of the telescope.

\subsection{Unresolved Emission lines}

There are some very narrow gaseous emission lines in our spectra. The spectra of DG Tau (Figure 11), DM Tau (Figure 4), and FS Tau (Figure 13) all show $12.8 \mu \mathrm{m}$ features arising from [NeII]. This can affect identification of the silica component, as noted by Sargent et al. (2008) with respect to the modeling by Sargent et al. (2006) of TW Hya. The presence of this feature in addition to a narrow feature at $\sim 12.4 \mu \mathrm{m}$ in the SL spectrum of TW Hya led Sargent et al. (2006) to conclude the presence of $\alpha$-quartz, which has a doublepeaked feature matching these two wavelengths. Investigation of high-resolution spectra of TW Hya showed that these two features belonged not to $\alpha$-quartz but to the Humphreys- $\alpha$ Hydrogen line (HI $\mathrm{n}=7-6)$ at $12.37 \mu \mathrm{m}$ and [NeII] at $12.81 \mu \mathrm{m}$.

$\mathrm{C}_{2} \mathrm{H}_{2}$ appears in the spectra of CoKu Tau/3 (Figure 6), DF Tau (Figure 11), and DL Tau (Figure 11). This emission line is located at the more innocuous wavelength of $13.7 \mu \mathrm{m}$ (Carr \& Najita 2008), and does not appear to have significantly affected the dust models of the spectra of these three objects. However, emission from HCN centered just longward of $14.0 \mu \mathrm{m}$ (Carr \& Najita 2008) is more problematic. Examples of spectra with this feature are AA Tau (Figure 12), BP Tau (Figure 12), and IT Tau (Figure 11). First, the feature occurs 
often in spectra taken with the SL and LL modules, which we splice together at precisely $14.00 \mu \mathrm{m}$. Often there is a flux level mismatch between SL and LL at this wavelength, making establishing the reality of this feature difficult. Second, the width of this feature varies. We have no dust component with an emission feature centered around $14 \mu \mathrm{m}$, so when the feature is wider, more data deviate from our model, worsening the model fit to the data. Thirdly, observations of evolved stars in the Large Magellanic Cloud (Sloan et al. 2006, 2008) sometimes show a feature at $\sim 14 \mu \mathrm{m}$ probably belonging to dust related to silicates. Lastly, there are emission features near these wavelengths in spectra of Calcium-Aluminum Inclusions in meteorites (Posch et al. 2007) and in laboratory spectra of the melilite solid series (Chihara et al. 2007), making identification of all of the $14 \mu \mathrm{m}$ features with HCN emission more ambiguous.

Finally, emission lines of $\mathrm{H}_{2} \mathrm{O}$ have been discovered in high resolution Spitzer IRS spectra of classical TTSs in Taurus, such as AA Tau (Carr \& Najita 2008). These lines are found in its SH and LH spectra, especially past $25 \mathrm{um}$. Many of these lines were found in the Spitzer IRS spectrum of the Class 0 YSO protostar NGC 1333 IRAS 4B (Watson et al. 2007). The lines are generally spread out over wavelength, but there are clumps of lines around 24.5-25.5, 27.5-28.5, and 30-31 $\mu \mathrm{m}$ wavelength. These water lines are unresolved, and our SH and LH spectra $(\mathrm{R} \sim 600)$ have been rebinned to SL/LL resolution $(\mathrm{R} \sim 90)$, so the effect of the lines generally being distributed over all wavelengths in $\mathrm{SH}$ and $\mathrm{LH}$ is to raise slightly the level of the continuum above the continuum underneath the water lines in the high resolution spectra. The clumps at 25, 28, and $30.5 \mu \mathrm{m}$ in the original high-resolution spectra, when

rebinned, will show up as very slight $\sim 0.5 \mu \mathrm{m}$ wide bumps in the rebinned spectra. We typically see no prominent crystalline silicate features centered at either 25 or $30.5 \mu \mathrm{m}$ in our spectra (though DH Tau has a small feature centered around $30.5 \mu \mathrm{m}$ wavelength that has been attributed to enstatite before; see Molster et al. 2002), but we do see many $28 \mu \mathrm{m}$ features in our data. Both the opacities of forsterite and enstatite have $28 \mu \mathrm{m}$ complexes, the $28 \mu \mathrm{m}$ complex of enstatite being more prominent with respect to its 23 and $33 \mu \mathrm{m}$ complexes, so the net effect of not accounting for the water emission might be obtaining a enstatite abundance slightly too large.

\subsection{Imperfect Extinction Correction}

Though we attempted to correct for extinction as explained in Section 2, two spectra proved to be difficult in this regard, those of FV Tau (Figure 13) and CoKu Tau/3 (Figure 6). For FV Tau, $\mathrm{A}_{V}=5.33$, and for $\mathrm{CoKu} \mathrm{Tau} / 3, \mathrm{~A}_{V}=5$. Both were just under the upper limit of $\mathrm{A}_{V}=6$ above which we would not correct for extinction and therefore not model, and 
both spectra show absorption at $15.2 \mu \mathrm{m}$ from $\mathrm{CO}_{2}$ ice; additionally, CoKu Tau/3 has ice absorption features at 6.0 and $6.8 \mu \mathrm{m}$ wavelengths as do heavily embedded Class I YSOs in Taurus-Auriga (Watson et al. 2004; Zasowski et al. 2007; Boogert et al. 2008). Though the model fit to the $10 \mu \mathrm{m}$ complex of $\mathrm{CoKu} \mathrm{Tau} / 3$ is pretty good, the same is not true for $\mathrm{FV}$ Tau. We suspect this to result from imperfect extinction correction. At longer wavelengths, however, the fit to FV Tau (excluding the region around the $15.2 \mu \mathrm{m} \mathrm{CO}_{2}$ ice absorption feature) is quite good, while the fit to the crystalline silicate complexes at 23 and $33 \mu \mathrm{m}$ is

only adequate. As any extinction correction at longer wavelengths is less than that for the $10 \mu \mathrm{m}$ silicate complex, we attribute this not to a problem with extinction correction, but rather to the opacity used to fit forsterite, which is discussed in the next subsection.

\section{Discussion}

\subsection{Inner versus Outer Disk Crystallinity and Grain Growth}

From the models for all 65 spectra, we computed the percentage of mass in a given dust grain species at one temperature out of all mass in dust at that temperature. With these mass percentages, we compute histograms displaying both warm and cool dust mass percentages. The histograms for total warm and cool large grain mass fraction, total warm and cool crystalline grain mass fraction, warm and cool forsterite mass fraction, warm and cool enstatite mass fraction, and warm and cool silica mass fraction are given in Fig. 13.

The test described and shown in Appendix B of fitting a $5 \mu \mathrm{m}$ radius heterogeneous grain profile with our standard model suggests that the abundance of crystalline grains could be underestimated by our standard models by up to $50 \%$. In addition, it also suggests the large grain abundance could be slightly underestimated by $23 \%$. The net effect of this bias is to underestimate the true crystalline and large grain abundances.

Figure 15a shows that the inner disk regions typically have much larger mass fraction in large grains than the outer disk regions. The mean and median in the histogram for warm large grains are around 50\%; for cool large grains, they are between 0 and 10 percent, though with a considerable "tail" up to $90 \%$. Noting that our $5 \mu \mathrm{m}$ radius porous grains are very similar in shape of opacity to $2 \mu \mathrm{m}$ solid grains (Sargent et al. 2006), our average and median warm large grain mass fractions of $44 \%$ and $45 \%$, respectively, compare favorably to the finding by Sicilia-Aguilar et al. (2007) of the average grain size for 1-2 Myr old systems being between 1.5 and $2 \mu \mathrm{m}$ radius (as probed by the $10 \mu \mathrm{m}$ feature). Our average and median cool large grain mass fraction were $17 \%$ and $0 \%$, respectively.

Figure 15b shows histograms of warm and cool crystalline dust mass fraction. Both 
histograms peak at low percentages of about $5 \%$ for warm crystalline dust and about $12 \%$ for cool crystalline dust (each of these percentages being the mode of the distribution), and there is considerable overlap. The cool crystalline dust histogram extends slightly to higher mass fraction bins, but we note the greater large grain mass fractions for the warmer inner disk regions. The average and median mass fractions for warm crystalline dust were $17 \%$ and $11 \%$, respectively, and the average and median mass fractions for cool crystalline dust were $23 \%$ and $15 \%$, respectively. Greater large grain mass fractions can lead to slight underestimation of the crystalline abundance, so the amount of crystallinity in the inner disk regions could be higher and closer to that of outer disk regions. Our findings of the mean, median, and mode of the warm crystalline dust mass fractions of $17 \%, 11 \%$, and $5 \%$ are consistent with those by Honda et al. (2006), but they are slightly less consistent with those by Sicilia-Aguilar et al. (2007). Honda et al. (2006) find typical warm crystalline dust mass fractions (as probed by the $10 \mu \mathrm{m}$ feature) typically to be between 5 and $20 \%$, regardless of system age, while Sicilia-Aguilar et al. (2007) find typical warm crystalline dust mass fractions to be between 5 and $7 \%$. Though crystalline mass fractions may be greater in the inner disk regions than outer disk regions (see Table 6), the total mass of the cool dust in our models is between $\sim 10$ and 1000 times greater than that of the warm dust in the models. The total mass in crystalline silicates is often greater for the cool crystalline dust than the warm crystalline dust, as Table 6 shows for V955 Tau. The crystalline mass fractions for warm and cool crystals are $24.7 \%$ and $20.6 \%$, respectively, being very similar. However, there is $\sim 250$ times more mass is cool dust than warm, so there is much more mass in $115 \mathrm{~K}$ crystalline dust than in $488 \mathrm{~K}$ crystalline dust according to the model.

Figures 15c, 15d, and 15e show the enstatite, forsterite, and silica mass fraction histograms. In Figure 15c, the warm enstatite mass fractions are very slightly displaced to larger values than the cool enstatite mass fractions. The previously discussed potential for slight underestimation of crystalline grain mass fraction for the inner disks due to greater grain growth reinforces our conclusion that the abundance of enstatite is greater in the inner disk regions. Figure 15d shows that both the warm and cool forsterite mass fraction histograms show a peak in the lowest bin; however, a large fraction of the cool forsterite distribution extends to greater mass fractions. First, we note the larger uncertainties in cool forsterite mass fractions than warm forsterite mass fractions (Table 6). Second, we once again note the potential to underestimate inner disk crystallinity. Together, these two considerations suggest the warm forsterite abundance is similar to that of cool forsterite for our sample. Figure 15e shows the same is true for silica as for forsterite; for the same reasons as forsterite, the warm and cool silica abundances in our sample are similar. The higher relative abundance of enstatite over forsterite in inner disk regions than for outer disk regions was also found by Bouwman et al. (2008). 


\subsection{Correlations}

In order to search for correlations between pairs of model parameters, stellar properties, and disk properties, we have computed the linear correlation coefficient, r (Bevington 1969), and its corresponding probability, $\mathrm{P}$, of finding a linear correlation coefficient of magnitude greater than or equal to these coefficients on a null data set, as above (Section 3.5). The correlation coefficient is weighted by uncertainties which are, for one data point, the square root of the sum of the squares of the uncertainties of the two quantities for which the correlation coefficient is being computed (see discussion on weighted least-squares fitting with uncertainties in both $\mathrm{x}$ and $\mathrm{y}$ by Bevington 1969). Note that scaling all dust mass weights by the same scalar less than unity would not change the correlation coefficient between two dust mass fractions. The uncertainty weighting of the correlation coefficient

shows up in both numerator and denominator of the correlation coefficient Bevington (1969) to the same power, so the scalar applied in the denominator would divide the scalar applied in the numerator to give 1 times the original correlation coefficient. Note that if $\mathrm{r}$ and the number, $\mathrm{N}$, of data points that are being tested for correlation do not change, $\mathrm{P}$ will not change either because $\mathrm{P}$ depends only upon $\mathrm{r}$ and $\mathrm{N}$. Values of $\mathrm{r}$ that significantly deviate from zero and values of $\mathrm{P}$ close to zero suggest significant correlation or anticorrelation. We deem as significant $\mathrm{P} \leq 2.0 \%$, resulting in $|\mathrm{r}| \geq 0.29$. Stellar masses, disk-to-star mass ratios, $\mathrm{n}_{6-13}$ and $\mathrm{n}_{13-31}$, and their uncertainties, plus multiplicity counts, stellar luminosities, and mass accretion rates used in this correlation analysis come from Watson et al. (2008). Submillimeter slopes were obtained from the study by Andrews \& Williams (2005).

First we explore trends between dust mass fractions. All trends between crystalline components are positive. Figure 16 shows a positive correlation between warm enstatite and warm forsterite with correlation coefficient $\mathrm{r}=0.62$ and probability $\mathrm{P}$ of having been drawn from a random distribution of $0.0 \%$. This trend is fairly clear, with a heavy concentration of points at low mass fractions and a collection of points with higher enstatite and forsterite mass fractions. Warm enstatite also has a correlation with warm silica, with $\mathrm{r}=0.31$ and $\mathrm{P}=1.2 \%$. Figure 17 shows a positive correlation between cool forsterite and cool silica, with $\mathrm{r}=0.29$ and $\mathrm{P}=2.0 \%$. Cool forsterite also correlates with warm large grain mass fraction $(\mathrm{r}=0.32$ and $\mathrm{P}=0.9 \%)$ and warm crystalline grain mass fraction $(\mathrm{r}=0.32$ and $\mathrm{P}=1.0 \%)$. The correlation between warm silica and cool crystalline (cool enstatite plus cool forsterite plus cool silica) mass fractions is shown in Figure 18, with $\mathrm{r}=0.32$ and $\mathrm{P}=0.9 \%$. This correlation is likely related to the correlation between warm silica and cool forsterite $(\mathrm{r}=0.36$ and $\mathrm{P}=0.4 \%)$. Warm enstatite also correlates with cool forsterite $(\mathrm{r}=0.29$ and $\mathrm{P}=1.9 \%)$. Cool forsterite mass fraction and submillimeter slope ( $\alpha$ reported by Andrews \& Williams 2005) correlate, with $\mathrm{r}=-0.40$ and $\mathrm{P}=2.0 \%$. Lower $\alpha$ means flatter submillimeter slope (flux density at submillimeter wavelengths, $\mathrm{F}_{\nu, s m m}$, is proportional to $\nu^{\alpha}$ ), implying that more growth of 
grains to millimeter sizes (instead of sizes of a few microns) accompanies a greater abundance of cool forsterite.

The general sense of these trends is that the crystalline dust abundances all correlate positively with each other. If the abundance of one type of crystal is high, it is likely that the abundances of the other two types will also be high. The general finding that crystal abundances track other crystal abundances has been noted before. Bouwman et al. (2001), via detailed modeling of the $10 \mu \mathrm{m}$ complexes of Herbig Ae/Be stars, noted correlation between forsterite and silica abundance in Herbig Ae/Be stars, and van Boekel et al. (2005) (using a similar analysis) noted correlation between enstatite abundance and total crystalline abundance in a similar population of Herbig Ae/Be stars. Watson et al. (2008) measured crystalline abundances using indices computed from ratios of flux integrated over small wavelength bands characteristic of emission from pyroxene, olivine, and silica at 10 and $33 \mu \mathrm{m}$ (they call these bands $\mathrm{P}_{10}, \mathrm{O}_{10}, \mathrm{~S}_{10}$, and $\mathrm{O}_{33}$, respectively); in their study, they found warm pyroxene correlated with warm silica and warm olivine, and that warm olivine correlated with warm silica and cool olivine. From this we conclude, as did Watson et al. (2008), that whatever produces the different kinds of crystals (forsterite, enstatite, and silica) produces them at a rate faster than the crystals can transform from one to another. As a specific example: if some silica forms by incongruent melting of enstatite, that amount of silica formed must be much less abundant than any silica formed by another means (e.g., via incongruent melting of amorphous pyroxene; Sargent et al. 2008). There is no significant correlation of crystalline silicate masses with disk-to-star mass ratio. A positive correlation of disk-to-star mass ratio might be expected for crystalline dust produced by shock annealing (Harker \& Desch 2002). The lack of this correlation does not necessarily mean shock annealing is not connected to dust processing, however, as disk mass estimates are based on assumed opacities of dust at submillimeter wavelengths. The amount by which these assumed opacities differ from actual opacities varies according to the extent of grain growth to millimeter sizes (see discussion by D'Alessio et al. 2006).

Furlan et al. (2006) computed and interpreted the SED continuum indices $\mathrm{n}_{6-13}$ and $\mathrm{n}_{13-25}$ as primarily measuring the degree of flaring of disks. Indices that are increasingly positive indicate increasingly flared disks, and more negative indices indicate increasingly flatter disks. The flattening of these disks was interpreted to mean that dust had settled from high in the disk atmosphere towards the disk midplane. Watson et al. (2008) used $\mathrm{n}_{13-31}$ instead of $\mathrm{n}_{13-25}$ to reduce contamination of the indices by the $20 \mu \mathrm{m}$ amorphous silicate feature. We use the $\mathrm{n}_{6-13}$ and $\mathrm{n}_{13-31}$ indices, interpreting them as primarily measuring the degree of flaring of disks (Furlan et al. 2006; Watson et al. 2008). We omit from our searches of correlation of parameters with disk continuum indices the points for the transitional and pre-transitional disks CoKu Tau/4 (D'Alessio et al. 2005), DM Tau and GM Aur 
(Calvet et al. 2005), and LkCa 15 (Espaillat et al. 2007). These four objects have spectra whose continuum colors are very red due not to highly flared disks but, rather, to the clearing of almost all small dust grains in the innermost regions of the disks. The disk indices for these four systems would measure how clear they are of such dust in their inner regions, so they are not included in the search for trends of parameters with disk indices.

Figure 19 illustrates a tight anticorrelation between warm crystalline grain abundance and $\mathrm{n}_{6-13}$, with $\mathrm{r}=-0.50$ and $\mathrm{P}=0.0 \%$. This can be explained by correlations between each of the three warm crystalline types of grains and $\mathrm{n}_{6-13}$. Warm forsterite and $\mathrm{n}_{6-13}$ are anticorrelated with $\mathrm{r}=-0.42$ and $\mathrm{P}=0.1 \%$, warm enstatite and $\mathrm{n}_{6-13}$ are correlated with $\mathrm{r}=-$ 0.41 and $\mathrm{P}=0.1 \%$, and warm silica and $\left.\mathrm{n}_{(} 6-13\right)$ are correlated with $\mathrm{r}=-0.39$ and $\mathrm{P}=0.2 \%$. Cool enstatite correlates with $\mathrm{n}_{13-31}(\mathrm{r}=-0.31$ and $\mathrm{P}=1.9 \%)$.

These findings are similar to those of Watson et al. (2008) that greater warm olivine and warm silica, measured by $\mathrm{O}_{10}$ and $\mathrm{S}_{10}$, respectively, anticorrelate with $\mathrm{n}_{6-13}$ and that greater warm enstatite, warm olivine, warm silica, and cool olivine (warm enstatite and cool olivine being measured by $\mathrm{P}_{10}$ and $\mathrm{O}_{33}$, respectively) anticorrelate with $\mathrm{n}_{13-31}$. The spectra described in Section 1.6 (low $10 \mu \mathrm{m}$ complex equivalent width) all indicate, with the exceptions of DG Tau and V807 Tau, highly settled systems with abundant crystalline silicates, though with relatively large uncertainties on these crystalline abundances. The settling of disks is interpreted here, as did Furlan et al. (2006) and Watson et al. (2008), as part of the evolution of protoplanetary disks. The processing of dust into crystalline silicates is also interpreted as evolution of such disks. Further, the models by Ciesla (2007) predict crystalline mass fraction should be correlated with dust sedimentation both in inner and outer disk regions. Therefore, the correlation of crystalline abundance with disk settling is expected as a general result of protoplanetary disk evolution.

Warm and cool large grain abundances both anticorrelate with $\mathrm{n}_{13-31}$. Figure 20 shows the correlation between warm large grain abundance and $\mathrm{n}_{13-31}(\mathrm{r}=-0.36$ and $\mathrm{P}=0.5 \%)$. This trend is interpreted as an increase in large grain abundance expected as a result of evolution of protoplanetary disks. Figure 21 shows that cool large grain abundance also anticorrelates with $\mathrm{n}_{13-31}(\mathrm{r}=-0.32$ and $\mathrm{P}=1.5 \%)$. Rettig et al. (2006) found other tentative evidence for dust settling with grain growth. Grain growth is, in fact, expected to be the cause of settling of dust in protoplanetary disks. From first-principles modeling, Weidenschilling (1997) find in his simulations of grain growth and settling towards disk midplane of protoplanetary disks that at 60,000 years of evolution in the disk (the initial diameter of their dust grains was 1 $\mu \mathrm{m})$, at $30 \mathrm{AU}$ from the central star they find particles with diameters from one $\mu \mathrm{m}$ to near one centimeter. The range of ages assumed for Class II YSOs in the Taurus-Auriga starforming region is 1-2 Myr, so substantial grain growth should have occurred for the 65 objects 
whose spectra are analyzed in this study. Also, at a disk age of $0.1 \mathrm{Myr}$, Weidenschilling (1997) predicts that at a disk radius of $30 \mathrm{AU}$, the dust-to-gas mass ratio at $6 \mathrm{AU}$ above the midplane should be $10^{-3}$ what it was at time zero, when the disk was initially wellmixed, at the same location in the disk. This ratio of the dust-to-gas mass ratio to its initial well-mixed value is what D'Alessio et al. (2006) call the settling parameter, $\epsilon$. As shown by Furlan et al. (2006), the disk indices of some of the Class II YSOs in the Taurus-Auriga star-forming region are consistent with such low values of $\epsilon$ (0.01-0.001). Bouwman et al. (2008) also find that the differing morphology of IRS spectra of YSOs are consistent with dust growth and settling. Large grain abundance anticorrelating with $\mathrm{n}_{13-31}$ is consistent with the expectation by Weidenschilling (1997) of increasing grain growth over time and also greater settling of larger dust grains towards the disk midplane.

Also significant is the result shown in Figure 15a that more large grains are found in inner disk regions than outer disk regions. The observations and models by van Boekel et al. (2004) required more large grains for disk regions inside 2 AU than for disk regions outside $2 \mathrm{AU}$ for spectra of two of the three Herbig Ae/Be stars they studied. Andrews \& Williams (2007) find that the surface density of protoplanetary disks decreases with radius in the disks as $\mathrm{r}^{-0.5}$ or $\mathrm{r}^{-1}$, so inner disk regions should be denser. This could encourage more rapid grain growth.

However, as noted previously, the correlations between large grain abundances and $\mathrm{n}_{13-31}$ are not very strong. One possible explanation for the weakness of correlation we find between warm large grain abundance and $n_{13-31}$ is that we are not measuring the true large grain abundance from each spectrum because we do not account for differing amorphousto-crystalline component ratios in the large inhomogeneous aggregate grains from system to system. The more crystalline components the average aggregate large grain in a given disk has, the less reliable a measure our large amorphous silicate grains are of the large grain extent in such disks (Min et al. 2008).

Another possibility to explain the weakness of trend of warm large grain abundance with $\mathrm{n}_{13-31}$ is that sufficiently high turbulence prevents rapid grain growth and settling of fewmicron-sized grains to the disk midplane (Dullemond \& Dominik 2004; Hubbard \& Blackman 2006). Perhaps different disks can have the same degree of flattening but differing abundances of large grains in the optically thin surface layer. Dullemond \& Dominik (2005) note that collisions replenish small grains. However, Ciesla (2007) finds that turbulence can also encourage collisions that facilitate grain growth. These large grains, once formed, can be stored in a dead zone underneath the turbulent live zone, and can be protected even if the dead zone is disturbed by the live zone above it (Ciesla 2007).

Warm large grain abundance also correlates with the known number of stars in the star 
system, with $\mathrm{r}=0.33$ and $\mathrm{P}=0.7 \%$, so perhaps multiplicity contributes to the dispersion seen in the trend between warm large grain abundance and $n_{13-31}$. Figure 22 shows a comparison of the histogram of warm large grain abundance for single star systems and the same for multiple star systems - one immediately notices the higher large grain mass fractions for multiple systems than single systems.

Finally, Figure 23 shows a fairly weak anticorrelation between warm large grain mass fraction and stellar mass, with $\mathrm{r}=-0.36$ and $\mathrm{P}=0.5 \%$. This may be related to the correlation between warm small amorphous grain mass fraction and stellar mass $(\mathrm{r}=0.40$ and $\mathrm{P}=0.1 \%)$. In our sample, the spectra of low mass stars indicate higher levels of warm large grain abundance than for higher-mass stars. Apai et al. (2005) found in their study of six brown dwarf spectra higher mass fractions of crystalline grains for lower mass stars. They concluded that the region giving rise to the $10 \mu \mathrm{m}$ feature was at smaller disk radii for less luminous, lower mass stars, and that in these innermost disk radii the crystalline dust abundance was higher. We interpret the trend of increasing warm (inner disk) large grain mass fraction with decreasing stellar mass similarly, in view of our finding of greater grain growth in inner disk regions than outer disk regions (Figure 15).

\section{Summary and Conclusions}

We have analyzed the dust composition of $65 \mathrm{~T}$ Tauri stars using spectra from the IRS on the Spitzer Space Telescope. These spectra show very prominent emission features and complexes at $10,16,19,23,28$, and $33 \mu \mathrm{m}$ wavelengths, which are characteristic of silica and silicate grains (both amorphous and crystalline) with sizes from submicron to a few microns. We have constructed spectral models for each of the spectra that include, firstly, blackbodies at two temperatures and, secondly, Planck functions at those two temperatures multiplied by scaled dust emissivities. These represent inner and outer disk emission from, firstly, the optically thick disk midplane and blackbody grains and, secondly, dust in the optically thin disk atmosphere with strong infrared resonances. The best fit for a given set of dust opacities is given by the pair of temperatures for which the global $\chi^{2}$ per degree of freedom is a minimum. We have tested the opacities on high-quality IRS spectra to find the best opacities to represent emission from submicron grains of enstatite, forsterite, silica, and amorphous silicates and large (few micron size) grains in the protoplanetary disks of the Taurus-Auriga association.

We conclude the following:

- High $\mathrm{S} / \mathrm{N}$ spectra suggest the best crystalline silicate opacities to use in modeling 
are those of iron-poor pyroxene (enstatite) and iron-poor olivine (forsterite). The best amorphous silicate opacities to use are those whose iron-to-magnesium ratios are nearly 1 (cosmic). This is consistent with the finding by Harker et al. (2002) that cometary amorphous silicates require significant iron in their compositions in order to heat to inferred dust temperatures. The adopted amorphous silicate closely matches the heating of Draine \& Lee (1984) "astronomical silicates".

- Though we use opacities of grains with $50 \%$ porosity and $5 \mu \mathrm{m}$ radius of both amorphous pyroxene and amorphous olivine, calculations of the covariance between these components in models of various high $\mathrm{S} / \mathrm{N}$ spectra show they are highly degenerate. This means our models cannot readily distinguish between the two species as large grains, but our models are more sensitive to the sum of their masses. Submicron amorphous pyroxene and submicron amorphous olivine are similarly degenerate but slightly less so. Cool silica is degenerate with cool submicron amorphous silicates as they share $20 \mu \mathrm{m}$ features. Cool enstatite and cool forsterite are somewhat degenerate because they share prominent 23,28 , and $33 \mu \mathrm{m}$ features (only somewhat because the $28 \mu \mathrm{m}$ feature of enstatite is relatively stronger than that of forsterite).

- A few spectra of T Tauri stars show evidence for extensive grain growth in that protoplanetary disks as indicated especially by their $10 \mu \mathrm{m}$ complexes.

- Though most spectra are fit satisfactorily using our truncated CDE forsterite opacity, a few are not. The deviations of the 10, 23, and $33 \mu \mathrm{m}$ features between our models and the spectra suggest olivine grains of greater size, greater porosity, greater iron content (namely, a nonzero iron content), or greater weighting towards extremely elongated or flattened grain shapes in the shape distribution are required, or some combination of these.

- Most spectra that require silica are fit well by the annealed silica opacity in the standard dust model mixture. The spectra of FZ Tau and DN Tau suggest a silica polymorph other than annealed silica (cristobalite and tridymite; see Fabian et al. 2000) would provide a better fit to their spectra. This would imply different cooling rates for silica, once formed, than implied by the presence of the higher temperature polymorphs of silica (Sargent et al. 2008). However, the $12.6 \mu \mathrm{m}$ features of these two exceptional spectra are only mildly inconsistent with the annealed silica in the dust models, so the suggestion of an alternative silica polymorph is weak.

- The models of the best enstatite exemplars, FN Tau and DH Tau, do not fit very well the strong crystalline silicate emission peaks at 28 and especially $33 \mu \mathrm{m}$, suggesting enstatite or forsterite dust at temperatures significantly lower than those used by our 
models is required by the data. It is unknown why such cold material is required to fit spectra indicating high enstatite abundance.

- Transitional and pre-transitional disks in this sample require negligible crystalline silicates and modest amounts of large grains (DM Tau being the exception, requiring significant large grains) to fit their spectra relative to the rest of the disks whose spectra were analyzed in this study. A similar (small) number of spectra of systems not known to be transitional or pre-transitional also require high abundances of submicron amorphous silicates.

- A few spectra have $10 \mu \mathrm{m}$ complexes that have small equivalent widths. Most of these are fit well by crystalline silicates as opposed to large grains, which is inconsistent with the idea that decreasing peak-to-continuum ratio of the $10 \mu \mathrm{m}$ complex necessarily implies grain growth.

- The goodness of fit of our models to the majority of our spectra means the opacities of large crystalline silicate grains are not typically required. This, in turn, implies that, if grains grow to larger sizes by agglomeration of grains of differing composition, then on average in the Taurus-Auriga YSO population the more abundant subcomponents of these heterogeneous aggregate large grains are amorphous rather than crystalline.

- Higher abundances of large grains are found in inner disk regions than outer disk regions, suggesting grain growth occurs more rapidly in inner disk regions.

- Crystalline silicate abundances are very similar in the inner and outer disk regions. This contrasts interestingly with the finding by van Boekel et al. (2004) that regions inside $2 \mathrm{AU}$ in disks around Herbig Ae/Be stars have much more processed dust than regions outside of 2 AU. F04147+2822 exemplifies the contrast, having prominent 19, 23, 28, and $33 \mu \mathrm{m}$ complexes but very little indication of crystallinity based on its $10 \mu \mathrm{m}$ complex. This suggests the inner region of this disk giving rise to the $10 \mu \mathrm{m}$ complex has a lower abundance of crystals than the outer region giving rise to emission past $19 \mu \mathrm{m}$ wavelength.

- Cool forsterite also correlates with flatter submillimeter SEDs, suggesting grain growth to millimeter sizes is more extensive for disks with more cool forsterite.

- Each crystalline silicate abundance always correlates positively and significantly with abundances of other crystalline silicate species. This suggests that whatever produces crystalline silicates does so at a faster rate than that at which any of the crystalline silicate species transform into any of the others. This, in turn, suggests that amorphous 
dust is processed into crystalline dust at a rate greater than one kind of crystalline silicate species can transform into another.

- Crystalline silicates in inner disk regions are more abundant for bluer, flatter disks, those with more advanced settling of dust grains towards disk midplane.

- Large grains in the inner disk regions are, on average, more abundant for more settled (bluer) disks. There is a fair degree of dispersion in the inner disk large grain abundance for a given degree of settling, suggesting different rates of settling of large grains in different disks. There is also an indication that the abundance of large grains in the inner disk correlates with known multiplicity of stellar system.

- The spectra of disks around less massive stars indicate higher abundances of large grains in their inner disk regions.

This work is based on observations made with the Spitzer Space Telescope, which is operated by the Jet Propulsion Laboratory, California Institute of Technology under NASA contract 1407. Support for this work was provided by NASA through Contract Number 1257184 issued by JPL/Caltech and through the Spitzer Fellowship Program, under award 011 808-001, and JPL contract 960803 to Cornell University, and Cornell subcontracts 314195714 to the University of Rochester. The authors wish to thank Harald Mutschke for sharing the opacity in tabular form of the annealed silica presented by Fabian et al. (2000). A.L. acknowledges support from the Chandra theory program, the Hubble theory programs, and the Spitzer theory programs. SMART was developed by the IRS Team at Cornell University and is available through the Spitzer Science Center at Caltech. This publication makes use of

the Jena-St. Petersburg Database of Optical Constants (Henning et al. 1999). The authors made use of the SIMBAD astronomical database and would like to thank those responsible for its upkeep.

\section{A. Error Bars and Mispointing}

We corrected for slight mispointing of the telescope from the standard nod positions by scaling the mispointed nods, as described by Sargent et al. (2008). For observations taken by the low spectral resolution modules SL and LL, only mispointing in the dispersion direction (perpendicular to the length of the slit) could give rise to loss of flux. Mispointing of the high resolution slits along the length of the slit (spatial direction) towards the center of the slit can actually result in obtaining more flux than is present if one does not consider the RSRFs 
used to calibrate flux were constructed from observations of flux standards at nominal nod positions. More flux enters through the slit if the target is positioned closer to the center of the slit than a nominal nod position (like the flux calibrator is); in this case, one must apply a scalar factor less than 1 to the affected nod spectrum to correct for the extra fraction of total signal from the target not measured for the calibrator. SH has a smaller slit and is therefore more sensitive to mispointing in either spectral or spatial directions; therefore, the relative amount of scalar correction is typically higher for $\mathrm{SH}$ than $\mathrm{LH}$. The scalars used are given in Table A1.

\section{B. Test of Large Grains with Heterogeneous Composition}

We conducted tests of the combination of Bruggeman EMT and Mie Theory used to compute effective complex dielectric constants and opacities for our large grains. Note that Sargent et al. (2006) found that opacity curves of sub-micron spherical $60 \%$ vacuum (porous) grains of forsterite and $\alpha$-quartz were identical to those computed for solid grains of the same materials in the CDE shape distribution. We have computed the effective complex dielectric function for material that is, by volume, $60 \%$ vacuum (complex dielectric function equal to unity), 20\% forsterite (complex dielectric function by Sogawa et al. 2006), and 20\% amorphous olivine $\mathrm{MgFeSiO}_{4}$ (complex dielectric function by Dorschner et al. 1995) as follows. First we computed using Bruggeman EMT the effective complex dielectric function of $50 \%$ amorphous olivine and $50 \%$ forsterite a-axis; we also computed the same effective complex dielectric functions for 50/50 mixtures of amorphous olivine and each of the other two forsterite crystallographic axes, b and c. Then we computed using Bruggeman EMT the effective complex dielectric constants for material that is $60 \%$ by volume vacuum and $40 \%$ the 50/50 mixture of amorphous olivine and forsterite; again, the same was done for the effective complex dielectric functions involving forsterite $\mathrm{b}$ and c axes. This resulted in 3 sets of complex dielectric functions, one for each of the 3 crystallographic axes of forsterite, of material that is, by volume, $60 \%$ vacuum, $20 \%$ forsterite, and $20 \%$ amorphous olivine. Next we computed opacities for each of the three sets of effective complex dielectric functions for Rayleigh-limit-size spheres of these materials. Finally, we averaged the three opacities together to account for anisotropy of optical constants (see Bohren \& Huffman 1983) for forsterite. We then calculated the flux density from 1 lunar mass of such grains 140 parsecs away at $300 \mathrm{~K} .1 \%$ relative uncertainties were assumed, which are about the best we can expect from our sample of 65 spectra.

The 7.7-37 $\mu \mathrm{m}$ part of this spectrum was then modeled using our standard opacities, which are listed in the notes for Table 6, with the exception of using the opacities of forsterite 
and amorphous olivine grains in the CDE shape distribution (instead of tCDE and CDE2). We used the CDE for these two dust types in anticipation of the porous-CDE correspondence reported by Sargent et al. (2006). In Figure A1, we show the fit of the model to this test spectrum. Although none of the dust opacities were precluded at the outset, the only opacities used were the CDE opacities for forsterite and amorphous olivine, with masses of 0.45 and $0.43 \mathrm{M}_{\text {Moon }}$, respectively. The fit is very good to all wavelengths, though we note deviations for the strongest features at 11.4, 19, and $23 \mu \mathrm{m}$; we also note that the correspondence between porous grain and CDE opacities reported by Sargent et al. (2006) was not perfect. The model used grains at 308K, only slightly different from the assumed $300 \mathrm{~K}$. This slightly higher than assumed temperature explains the slightly lower $\left(0.88 \mathrm{M}_{\text {Moon }}\right)$ than assumed $\left(1 \mathrm{M}_{\text {Moon }}\right.$ ) total mass of dust. We take this as support for the conclusion by Min et al. (2008) that opacities of aggregates can be expressed as a sum of the opacities of homogeneous grains composed of the same materials as the aggregate's components, as applied to heterogeneous aggregates in the Rayleigh size limit.

Next we conducted a similar test, but for large aggregates. We used the same effective complex dielectric function of vacuum, forsterite, and amorphous olivine in a 60/20/20 volume ratio as before but this time to compute opacities for $5 \mu \mathrm{m}$ radius grains of the material. We computed the test spectrum assuming 1 lunar mass of grains at 300K located 140 pc away. Here, we used our standard opacities exactly as listed in the notes for Table 6, to gauge the effect of large grains in protoplanetary disks being aggregates of both amorphous and crystalline grains. Dust at only one temperature was allowed, but no dust species at this temperature was precluded from the model at the outset. This time, the best-fit model dust temperature is lower, $264 \mathrm{~K}$, and enstatite is used in addition to forsterite and large ( $5 \mu \mathrm{m}$ radius porous) grains of amorphous olivine, though at a lower significance level than either the amorphous olivine or forsterite. We show this model in Figure A2. The total mass of the dust used by the model, 1.25 lunar masses, is a sum of $0.96 \mathrm{M}_{\text {Moon }}$ of large amorphous olivine plus $0.20 \mathrm{M}_{\text {Moon }}$ of forsterite, $0.09 \mathrm{M}_{\text {Moon }}$ of enstatite, and $0.001 \mathrm{M}_{\text {Moon }}$ of submicron amorphous pyroxene, which is somewhat greater than the mass assumed for the aggregates. This increase in mass was needed to compensate for the lower temperature. The model becomes an increasingly poorer fit at increasing wavelengths. Part of this failure may be due to the use of the restricted shape distribution (tCDE) for the forsterite instead of the more extreme shape distribution CDE (used previously to test the Rayleighlimit aggregate). An additional population of forsterite grains at a lower temperature than used may improve the fit. This suggests any serious failures of our standard model to fit spectra with prominent crystalline silicate features may imply a significant amount of grain growth of aggregate grains with significant crystalline silicate abundances (here, $50 \%$ of the solid mass of the aggregate). This test also implies that the crystalline abundance could be 
underestimated by $50 \%$ (0.29/1.25 instead of $0.625 / 1.25$ fractional abundance of crystalline silicates in heterogeneous aggregates) of the true value, and it implies that the large grain abundance could be slightly underestimated (0.96 instead of 1.25 lunar masses of large (5 $\mu$ m radius) grains) by $100 \times(0.29 / 1.25) \%=23 \%$.

\section{Importance of Large Grain Opacity}

We tested the necessity of using opacities of large grains in our models. We used our standard dust model on IS Tau, one of the objects whose spectrum was modeled using a significant amount of large grains, but we eliminated the opacities of large amorphous pyroxene and large amorphous olivine in the fit. The resulting model, shown in Figure $\mathrm{A} 3$, is a poorer fit to the $10 \mu \mathrm{m}$ complex, not "filling out" the full width of the $10 \mu \mathrm{m}$ complex. Reduced $\chi^{2}$ for this large-grains-precluded model was 4.9, which is 1.6 higher than the reduced $\chi^{2}$ of 3.3 for the model with large grains. We conclude the opacities of large amorphous grains are required in our models.

\section{Porosity Test}

We tested how sensitive our models are to the exact combination of porosity and grain size when we compute and use opacities of large grains in our models to detect grain growth. Instead of $5 \mu \mathrm{m}$ radius $60 \%$ porous grains, we computed opacities for $20 \mu \mathrm{m}$ radius grains that are $88 \%$ porous. We chose this size to preserve the optical depth through the center of

the grain. As noted by Sargent et al. (2006), $5 \mu \mathrm{m}$ radius $60 \%$ porous grains were chosen to preserve the optical depth through the center of the $2 \mu \mathrm{m}$ solid grains of amorphous olivine used by Bouwman et al. (2001) to represent grain growth in modeling spectra of Herbig Ae/Be stars. This preservation of the optical depth through the center of the grain resulted in a close correspondence of the opacities of $5 \mu \mathrm{m}$ radius porous grains and of $2 \mu \mathrm{m}$ radius solid grains. Although $90 \%$ vacuum (by volume) grains would be the logical extension $88 \%$ vacuum $20 \mu \mathrm{m}$ radius grains proved to result in an opacity most like that of $5 \mu \mathrm{m}$ radius $60 \%$ vacuum grains. As a test of this opacity, we modeled the spectrum of UZ Tau/e, noted in Section 4 to require a large amount of large grains; here, we replace the opacity of $5 \mu \mathrm{m}$ $60 \%$ porous grains of amorphous olivine with that of $20 \mu \mathrm{m} 88 \%$ porous grains of amorphous olivine. We show the best-fit model in Figure A4, compared to the model shown in Figure 4 and described in Section 4. The fit is not much different, and the reduced $\chi^{2}$ is 5.6 instead of the 5.4 obtained with our standard opacities. Therefore, we cannot make precise statements on grain size and porosity individually for a given protoplanetary disk based only on the IRS 
spectra.

\section{REFERENCES}

Andrews, S. M., \& Williams, J. P. 2005, ApJ, 631, 1134

Andrews, S. M., \& Williams, J. P. 2007, ApJ, 659, 705

Apai, D., Pascucci, I., Bouwman, J., Natta, A., Henning, T., \& Dullemond, C. P. 2005, Science, 310, 834

Armus, L., et al. 2007, ApJ, 656, 148

Avni, Y. 1976, ApJ, 210, 642

Batalha, C. C., et al. 1998, A\&AS, 128, 561

Bevington, P. R. 1969, New York: McGraw-Hill, 1969,

Bohren, C. F., \& Huffman, D. R. 1983, Absorption and Scattering of Light by Small Particles, New York: Wiley

Boogert, A. C. A., et al. 2008, ApJ, 678, 985

Bouwman, J., Meeus, G., de Koter, A., Hony, S., Dominik, C., \& Waters, L. B. F. M. 2001, A\&A, 375, 950

Bouwman, J., de Koter, A., Dominik, C., \& Waters, L. B. F. M. 2003, A\&A, 401, 577

Bouwman, J., et al. 2008, ArXiv e-prints, 802, arXiv:0802.3033

Bowey, J. E., \& Adamson, A. J. 2002, MNRAS, 334, 94

Bradley, J. 2003, LNP Vol. 609: Astromineralogy, 609, 217

Calvet, N., et al. 2005, ApJ, 630, L185

Cambresy, L., Copet, E., Epchtein, N., de Batz, B., Borsenberger, J., Fouque, P., Kimeswenger, S., \& Tiphene, D. 1998, A\&A, 338, 977

Carr, J. S., \& Najita, J. R. 2008, Science, 319, 1504

Chen, C. H., et al. 2006, ApJS, 166, 351 
Chiang, E. I., \& Goldreich, P. 1997, ApJ, 490, 368

Chiar, J. E., et al. 2007, ApJ, 666, L73

Chihara, H., Koike, C., \& Tsuchiyama, A. 2001, PASJ, 53, 243

Chihara, H., Koike, C., Tsuchiyama, A., Tachibana, S., \& Sakamoto, D. 2002, A\&A, 391, 267

Chihara, H., Koike, C., \& Tsuchiyama, A. 2007, A\&A, 464, 229

Ciesla, F. J. 2007, ApJ, 654, L159

Cohen, M., \& Witteborn, F. C. 1985, ApJ, 294, 345

Cohen, M., Megeath, S. T., Hammersley, P. L., Martín-Luis, F., \& Stauffer, J. 2003, AJ, 125,2645

Cox, A. N. 2000, Allen's Astrophysical Quantities,

Crovisier, J., Leech, K., Bockelee-Morvan, D., Brooke, T. Y., Hanner, M. S., Altieri, B., Keller, H. U., \& Lellouch, E. 1997, Science, 275, 1904

D’Alessio, P., Calvet, N., \& Hartmann, L. 2001, ApJ, 553, 321

D'Alessio, P., et al. 2005, ApJ, 621, 461

D'Alessio, P., et al. 2006, ApJ, 638, 314

Day, K. L. 1979, ApJ, 234, 158

Dorschner, J., Friedemann, C., Guertler, J., \& Henning, T. 1988, A\&A, 198, 223

Dorschner, J., Begemann, B., Henning, T., Jaeger, C., \& Mutschke, H. 1995, A\&A, 300, 503

Draine, B. T., \& Lee, H. M. 1984, ApJ, 285, 89

Draine, B. T. 2003, ARA\&A, 41, 241

Dullemond, C. P., \& Dominik, C. 2004, A\&A, 421, 1075

Dullemond, C. P., \& Dominik, C. 2005, A\&A, 434, 971

Espaillat, C., et al. 2007, ApJ, 664, L111 
Espaillat, C., Calvet, N., D’Alessio, P., Hernández, J., Qi, C., Hartmann, L., Furlan, E., \& Watson, D. M. 2007, ApJ, 670, L135

Espaillat, C., et al. 2008, submitted

Fabian, D., Jäger, C., Henning, T., Dorschner, J., \& Mutschke, H. 2000, A\&A, 364, 282

Fabian, D., Henning, T., Jäger, C., Mutschke, H., Dorschner, J., \& Wehrhan, O. 2001, A\&A, 378,228

Forrest, W. J., Gillett, F. C., \& Stein, W. A. 1975, ApJ, 195, 423

Forrest, W. J., Houck, J. R., \& Reed, R. A. 1976, ApJ, 208, L133

Furlan, E., et al. 2006, ApJS, 165, 568

Furlan, E., et al. 2007, ApJ, 664, 1176

Glassgold, A. E., Najita, J., \& Igea, J. 2004, ApJ, 615, 972

Gorti, U., \& Hollenbach, D. 2008, ArXiv e-prints, 804, arXiv:0804.3381

Harker, D. E., \& Desch, S. J. 2002, ApJ, 565, L109

Harker, D. E., Wooden, D. H., Woodward, C. E., \& Lisse, C. M. 2002, ApJ, 580, 579

Henning, T., Il'In, V. B., Krivova, N. A., Michel, B., \& Voshchinnikov, N. V. 1999, A\&AS, 136,405

Higdon, S. J. U., et al. 2004, PASP, 116, 975

Holweger, H., Heise, C., \& Kock, M. 1990, A\&A, 232, 510

Honda, M., Kataza, H., Okamoto, Y. K., Miyata, T., Yamashita, T., Sako, S., Takubo, S., \& Onaka, T. 2003, ApJ, 585, L59

Honda, M., et al. 2006, ApJ, 646, 1024

Houck, J. R., et al. 2004, ApJS, 154, 18

Hubbard, A., \& Blackman, E. G. 2006, New Astronomy, 12, 246

Jaeger, C., Mutschke, H., Begemann, B., Dorschner, J., \& Henning, T. 1994, A\&A, 292, 641

Jaeger, C., Molster, F. J., Dorschner, J., Henning, T., Mutschke, H., \& Waters, L. B. F. M. 1998, A\&A, 339, 904 
Kamp, I., \& Dullemond, C. P. 2004, ApJ, 615, 991

Kastner, J. H., Buchanan, C. L., Sargent, B., \& Forrest, W. J. 2006, ApJ, 638, L29

Kemper, F., Vriend, W. J., \& Tielens, A. G. G. M. 2004, ApJ, 609, 826 (erratum: 633, 534)

Kenyon, S. J., Dobrzycka, D., \& Hartmann, L. 1994, AJ, 108, 1872

Kenyon, S. J., \& Hartmann, L. 1995, ApJS, 101, 117

Kessler, M. F., et al. 1996, A\&A, 315, L27

Kessler-Silacci, J. E., Hillenbrand, L. A., Blake, G. A., \& Meyer, M. R. 2005, ApJ, 622, 404

Kessler-Silacci, J., et al. 2006, ApJ, 639, 275

Koike, C., et al. 2000, A\&A, 363, 1115

Koike, C., Chihara, H., Tsuchiyama, A., Suto, H., Sogawa, H., \& Okuda, H. 2003, A\&A, 399,1101

Li, A., \& Greenberg, J. M. 1998, ApJ, 498, L83

Li, A., \& Lunine, J. I. 2003, ApJ, 590, 368

Li, A., \& Lunine, J. I. 2003, ApJ, 594, 987

Li, A., Lunine, J. I., \& Bendo, G. J. 2003, ApJ, 598, L51

Luhman, K. L. 2004, ApJ, 602, 816

Marshall, J. A., Herter, T. L., Armus, L., Charmandaris, V., Spoon, H. W. W., BernardSalas, J., \& Houck, J. R. 2007, ApJ, 670, 129

McClure, M. K., et al. 2008, accepted

Min, M., Hovenier, J. W., Waters, L. B. F. M., \& de Koter, A. 2008, ArXiv e-prints, 806, arXiv:0806.4038

Miyake, K., \& Nakagawa, Y. 1993, Icarus, 106, 20

Molster, F. J., Waters, L. B. F. M., \& Tielens, A. G. G. M. 2002, A\&A, 382, 222

Pilipp, W., Hartquist, T. W., Morfill, G. E., \& Levy, E. H. 1998, A\&A, 331, 121

Posch, T., Mutschke, H., Trieloff, M., \& Henning, T. 2007, ApJ, 656, 615 
Press, W. H., Teukolsky, S. A., Vetterling, W. T., \& Flannery, B. P. 1992, Cambridge: University Press, - c1992, 2nd ed.,

Przygodda, F., van Boekel, R., Àbrahàm, P., Melznikov, S. Y., Waters, L. B. F. M., \& Leinert, C. 2003, A\&A, 412, L43

Rettig, T., Brittain, S., Simon, T., Gibb, E., Balsara, D. S., Tilley, D. A., \& Kulesa, C. 2006, ApJ, 646, 342

Sargent, B., et al. 2006, ApJ, 645, 395

Sargent, B., et al. 2008, accepted for publication by the Astrophysical Journal

Schegerer, A., Wolf, S., Voshchinnikov, N. V., Przygodda, F., \& Kessler-Silacci, J. E. 2006, A\&A, 456, 535

Schütz, O., Meeus, G., \& Sterzik, M. F. 2005, A\&A, 431, 165

Scott, A., \& Duley, W. W. 1996, ApJS, 105, 401

Sicilia-Aguilar, A., Hartmann, L. W., Watson, D., Bohac, C., Henning, T., \& Bouwman, J. 2007, ApJ, 659, 1637

Sloan, G. C., Devost, D., Bernard-Salas, J., Wood, P. R., \& Houck, J. R. 2006, ApJ, 638, 472

Sloan, G. C., Kraemer, K. E., Wood, P. R., Zijlstra, A. A., Bernard-Salas, J., Devost, D., \& Houck, J. R. 2008, arXiv:0807.2998

Snow, T. P., \& Witt, A. N. 1995, Science, 270, 1455

Sogawa, H., Koike, C., Chihara, H., Suto, H., Tachibana, S., Tsuchiyama, A., \& Kozasa, T. 2006, A\&A, 451, 357

Taylor, J. R. 1982, A Series of Books in Physics, Oxford: University Press, and Mill Valley: University Science Books, 1982,

Uchida, K. I., et al. 2004, ApJS, 154, 439

van Boekel, R., Waters, L. B. F. M., Dominik, C., Bouwman, J., de Koter, A., Dullemond, C. P., \& Paresce, F. 2003, A\&A, 400, L21

van Boekel, R., et al. 2004, Nature, 432, 479 
van Boekel, R., Min, M., Waters, L. B. F. M., de Koter, A., Dominik, C., van den Ancker, M. E., \& Bouwman, J. 2005, A\&A, 437, 189

Watson, D. M., et al. 2004, ApJS, 154, 391

Watson, D. M., et al. 2007, Nature, 448, 1026

Watson, D. M., et al. 2008, in preparation

Weidenschilling, S. J. 1997, Icarus, 127, 290

Werner, M. W., et al. 2004, ApJS, 154, 1

Wooden, D. H., Harker, D. E., Woodward, C. E., Butner, H. M., Koike, C., Witteborn, F. C., \& McMurtry, C. W. 1999, ApJ, 517, 1034

Wooden, D. H., Harker, D. E., \& Brearley, A. J. 2005, Chondrites and the Protoplanetary Disk, 341, 774

Zasowski, G., Markwick-Kemper, F., Watson, D. M., Furlan, E., Bohac, C. J., Hull, C., \& Green, J. D. 2007, arXiv:0712.2458

Zubko, V. G., Mennella, V., Colangeli, L., \& Bussoletti, E. 1996, MNRAS, 282, 1321 


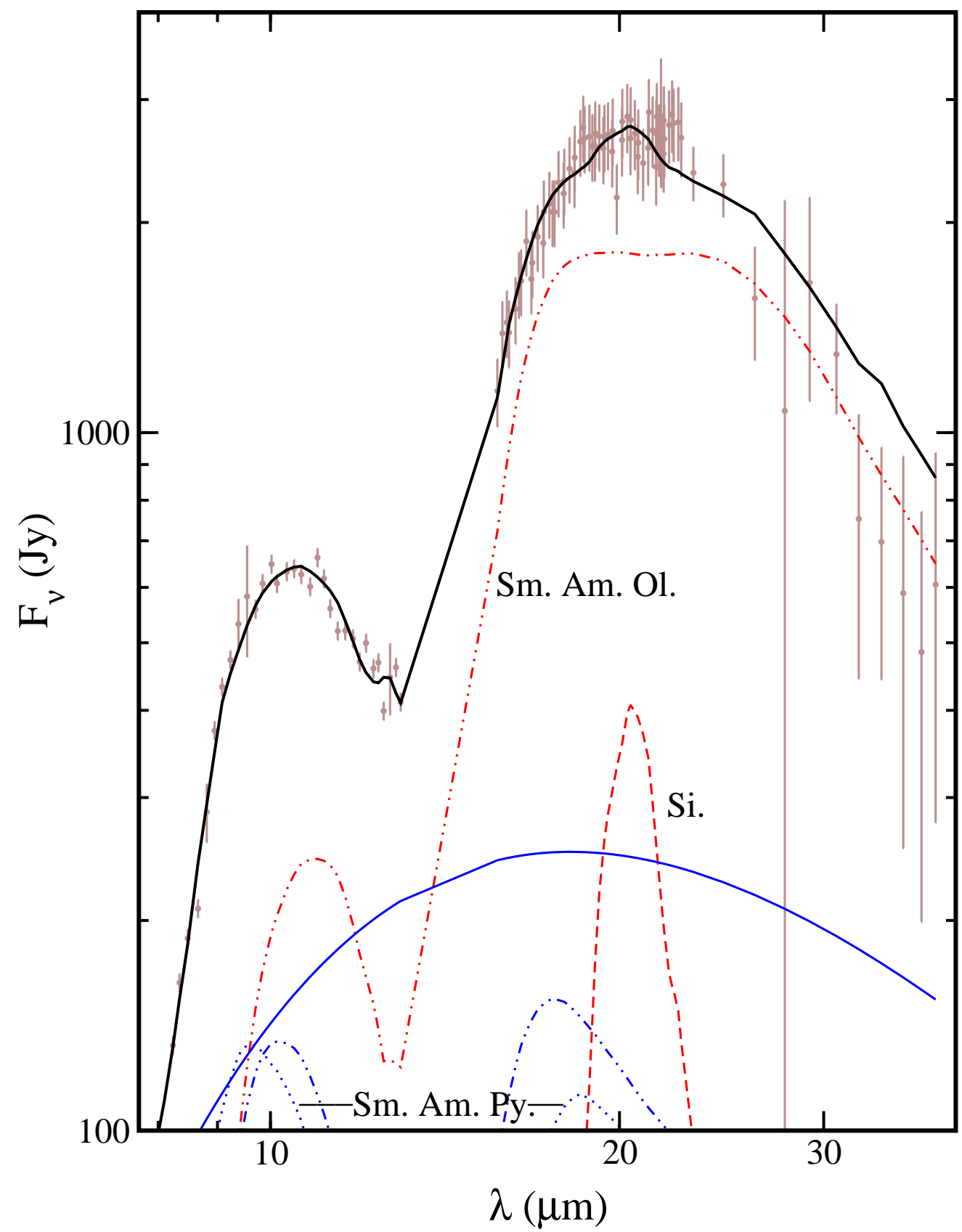

Fig. 1.- Model of the spectrum of the Trapezium. Model components are at bottom. Blue lines represent model components at higher temperature, red lines represent model components at lower temperature. Solid lines are blackbodies, dotted lines are submicron amorphous pyroxene, dash-double-dotted lines are submicron amorphous olivine, dot-longdash lines are large amorphous pyroxene, dot-double-dash lines are large amorphous olivine, dot-short-dash lines are enstatite, long-dashed lines are forsterite, and short-dashed lines are silica. 


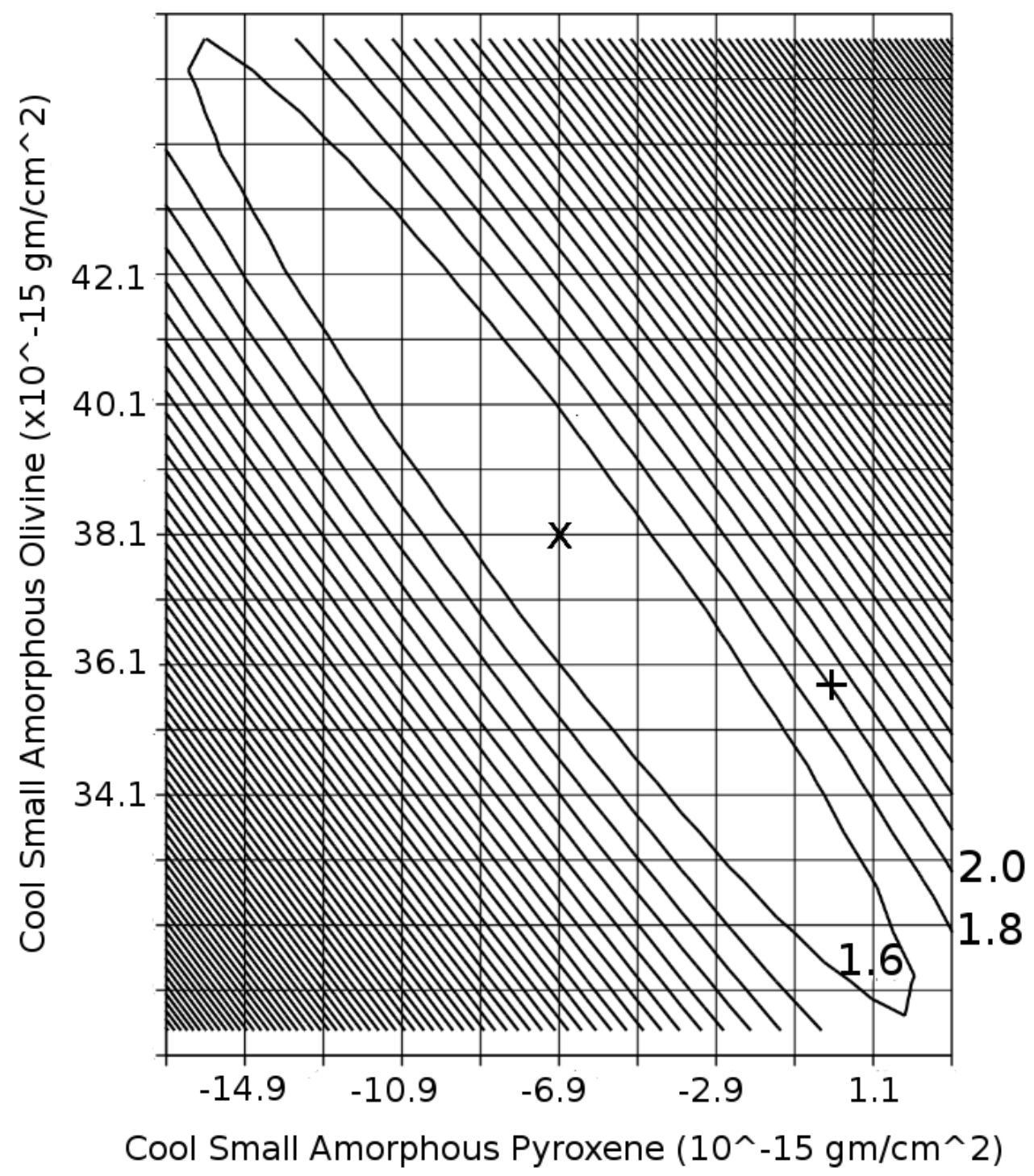

Fig. 2.- Contours of $\chi^{2}$ per degree of freedom for models of the Trapezium spectrum with dust at the same temperatures as the model shown in Figure 1, but with dust weights allowed to be negative (the "negative-allowed" model). The first three contours of reduced $\chi^{2}$ are labeled. The " $\mathrm{x}$ " gives the best-fit values of cool small amorphous pyroxene and cool small amorphous olivine for this model, resulting in reduced $\chi^{2}$ of 1.4 . The "+" gives the best-fit values of the same two dust components for the standard model (the "negative-not-allowed" model) shown in Figure 1, resulting in reduced $\chi^{2}$ of 1.6. This value, 1.6, is lower than that indicated on the plot, 2.0, because in the negative-not-allowed model, the mass weights of the other dust components were allowed to vary to obtain the global minimum in reduced $\chi^{2}$, while in the negative-allowed model they were not. Note that nearly the same minimal level of reduced $\chi^{2}$ can be obtained by replacing a given amount of one of the two dust components by about the same mass of the other. 


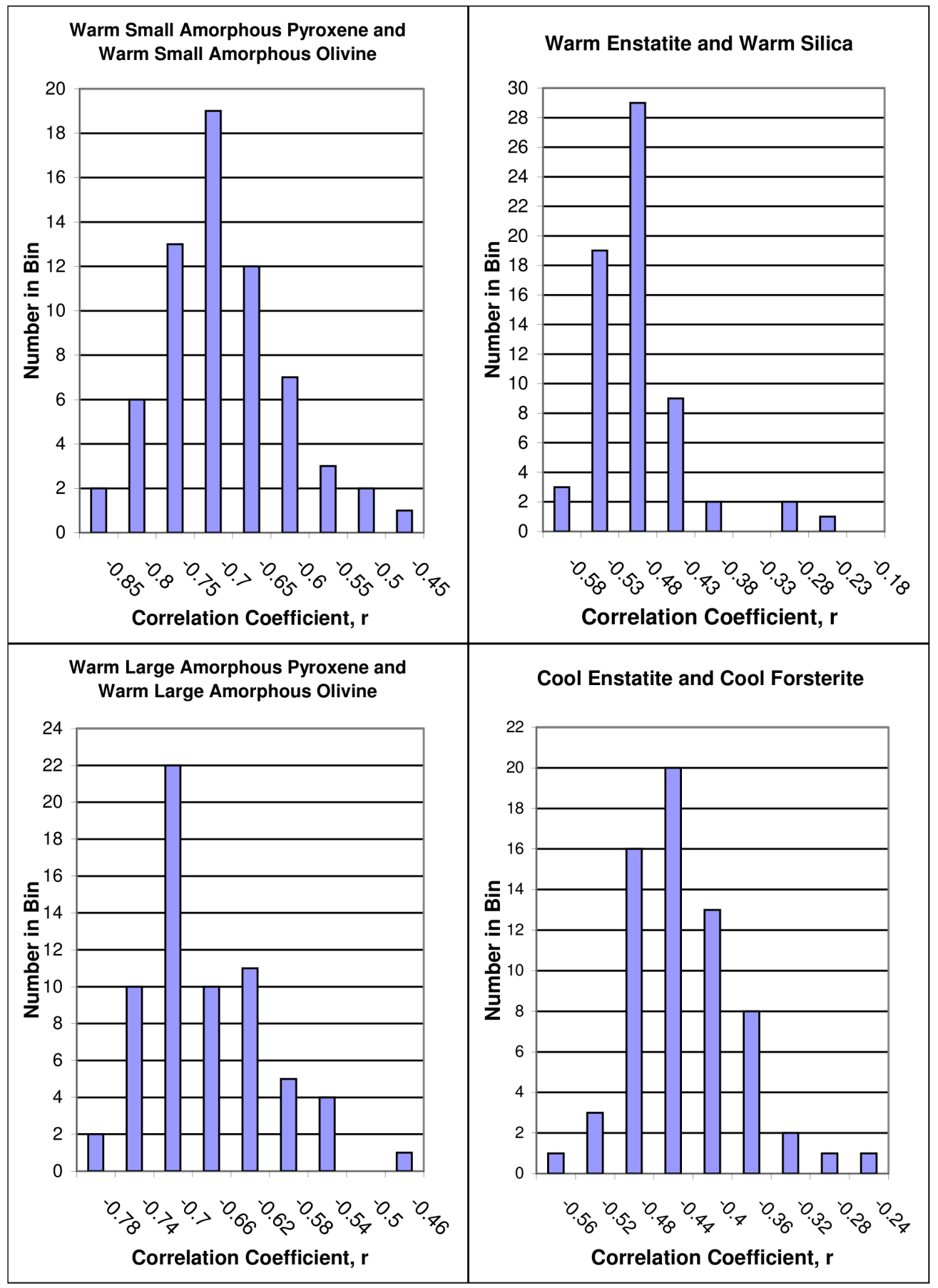

Fig. 3.- Histograms of the correlation coefficients obtained for all 65 spectra of TaurusAuriga T Tauri stars analyzed in this study, for four representative pairs of dust components. Varying degrees of degeneracy, or anticorrelation, of components are seen, with the most degeneracy coming from pairs of amorphous silicate grains at the same temperature. 


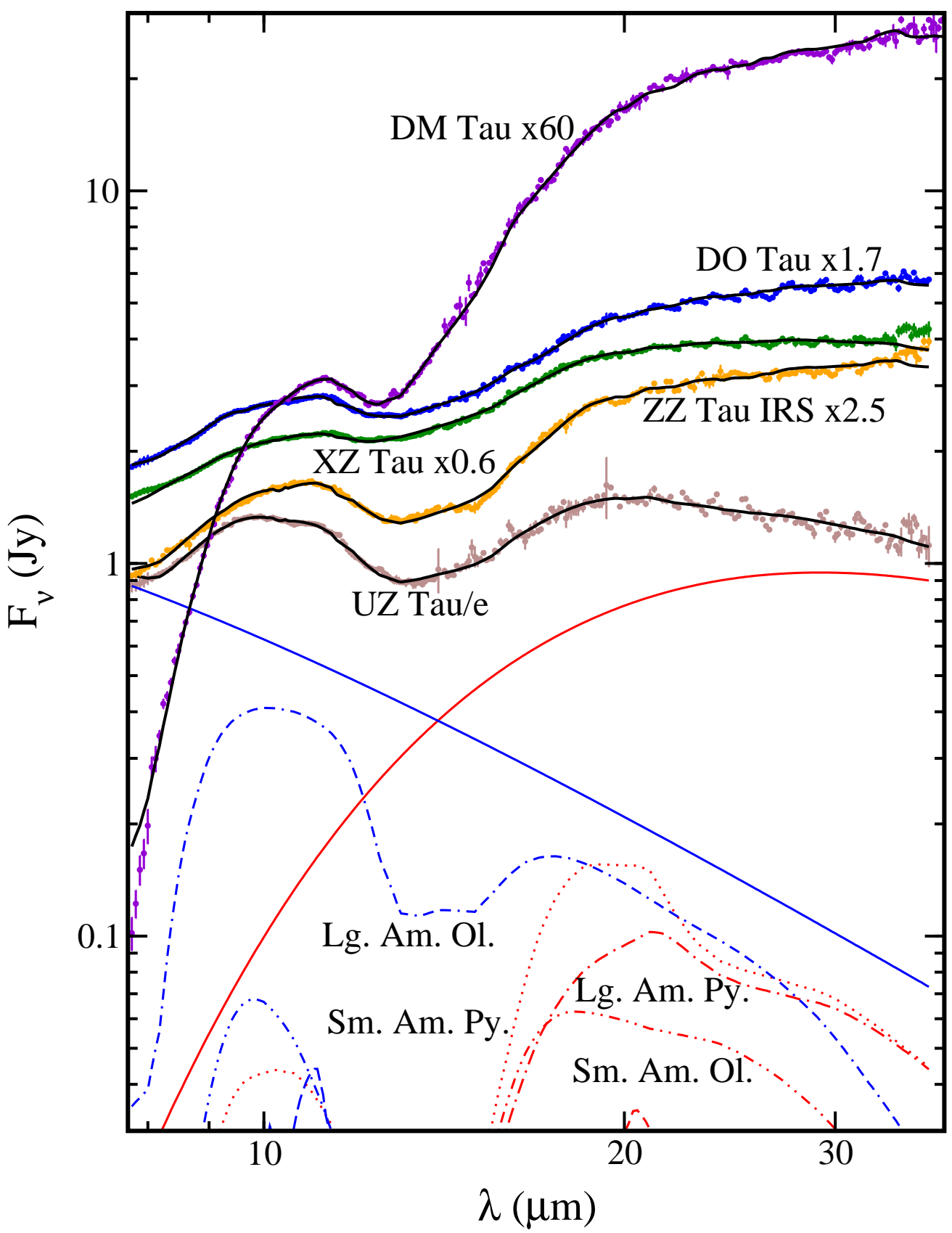

Fig. 4.- Spectra indicating substantial inner disk grain growth. Model components for model of UZ Tau/e are at bottom; blue lines represent model components at higher temperature, red lines represent model components at lower temperature. Solid lines are blackbodies, dotted lines are small (submicron) amorphous pyroxene, dash-double-dotted lines are small (submicron) amorphous olivine, dot-long-dash lines are large amorphous pyroxene, dot-double-dash lines are large amorphous olivine, dot-short-dash lines are enstatite, long-dashed lines are forsterite, and short-dashed lines are silica. 


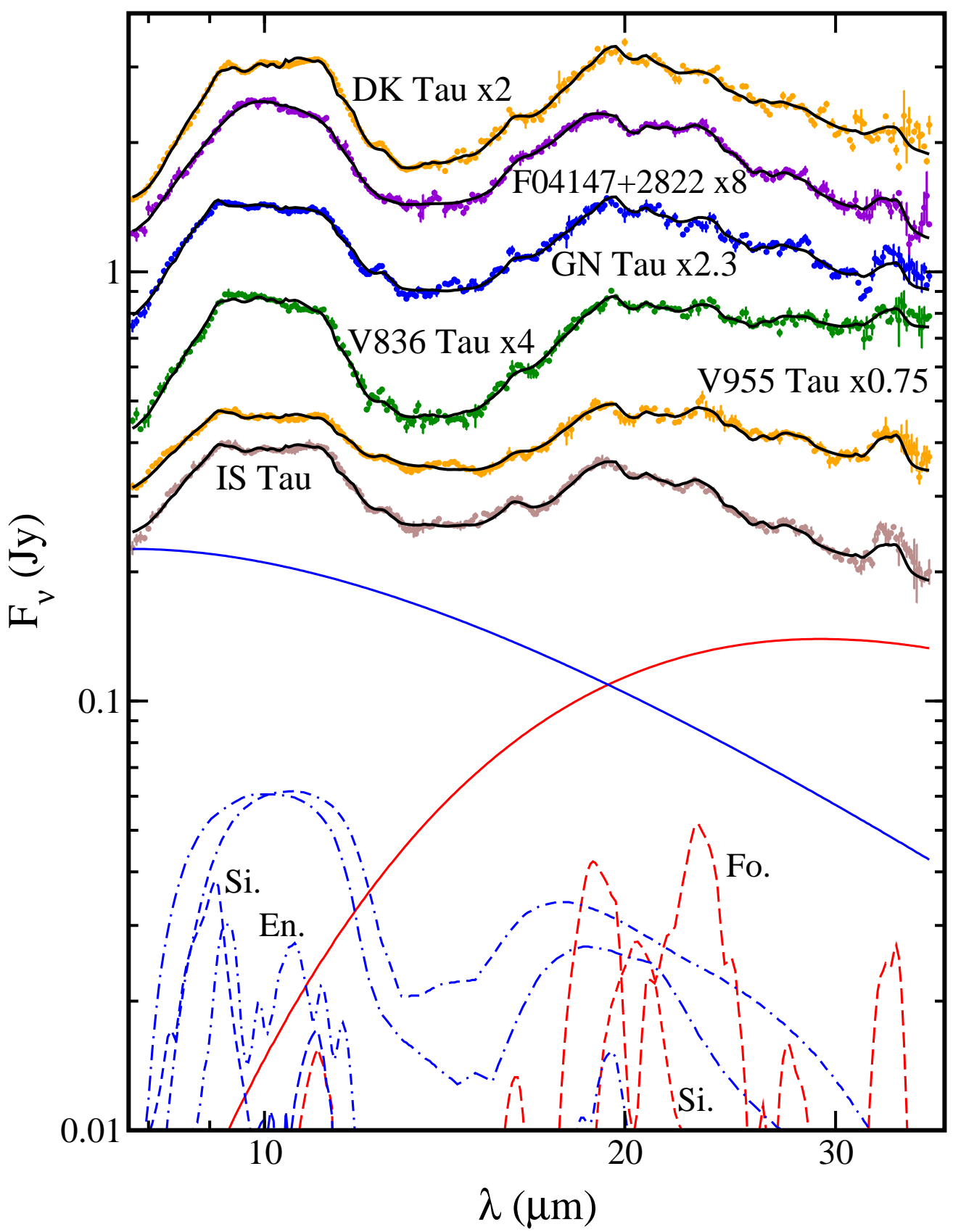

Fig. 5.- Spectra with prominent forsterite features. Model components are shown for IS Tau, same style and color convention as Figure 4. 


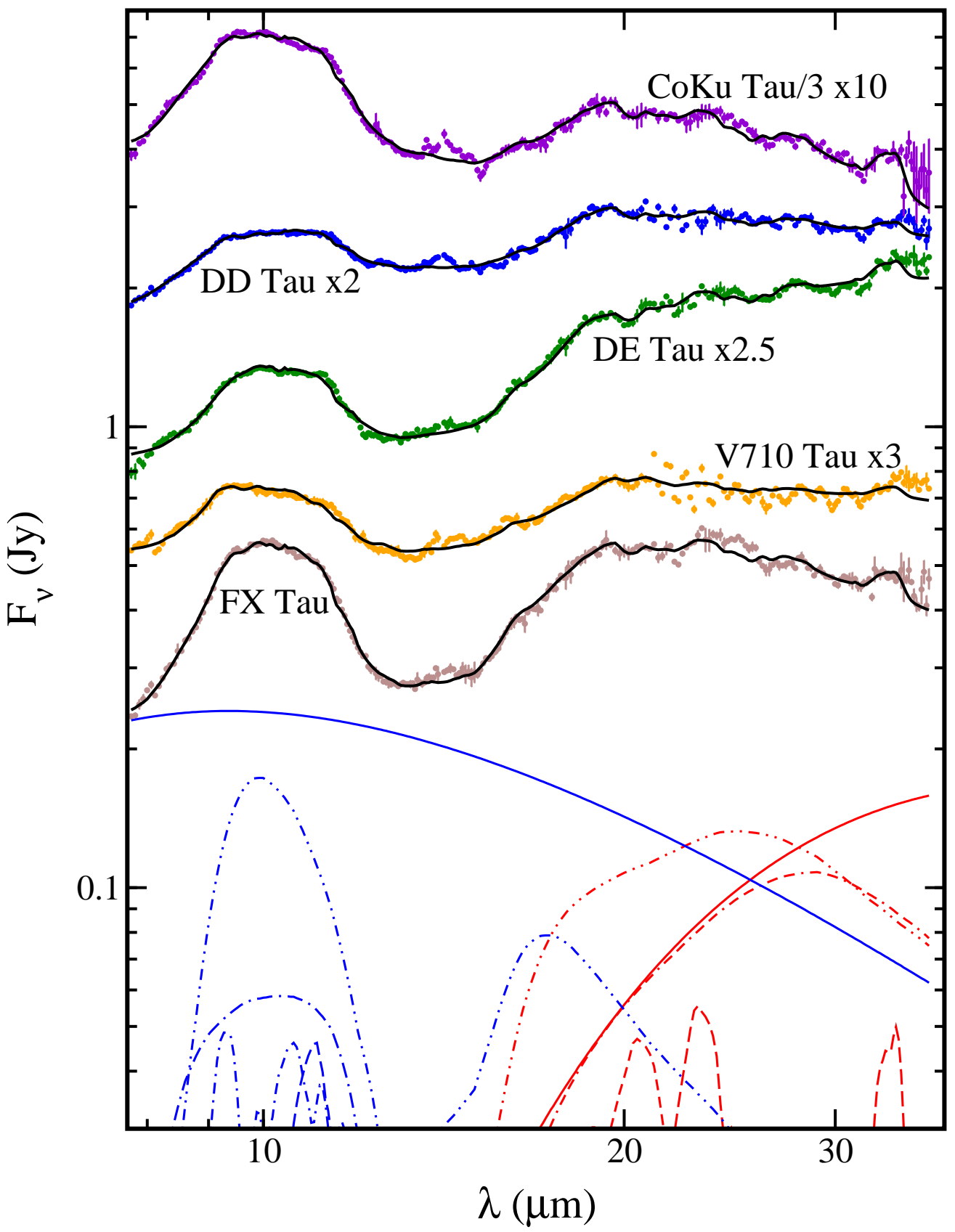

Fig. 6.- Spectra indicating forsterite, but not fit well by our forsterite opacity. Model components are shown for FX Tau, same style and color convention as Figure 4. 


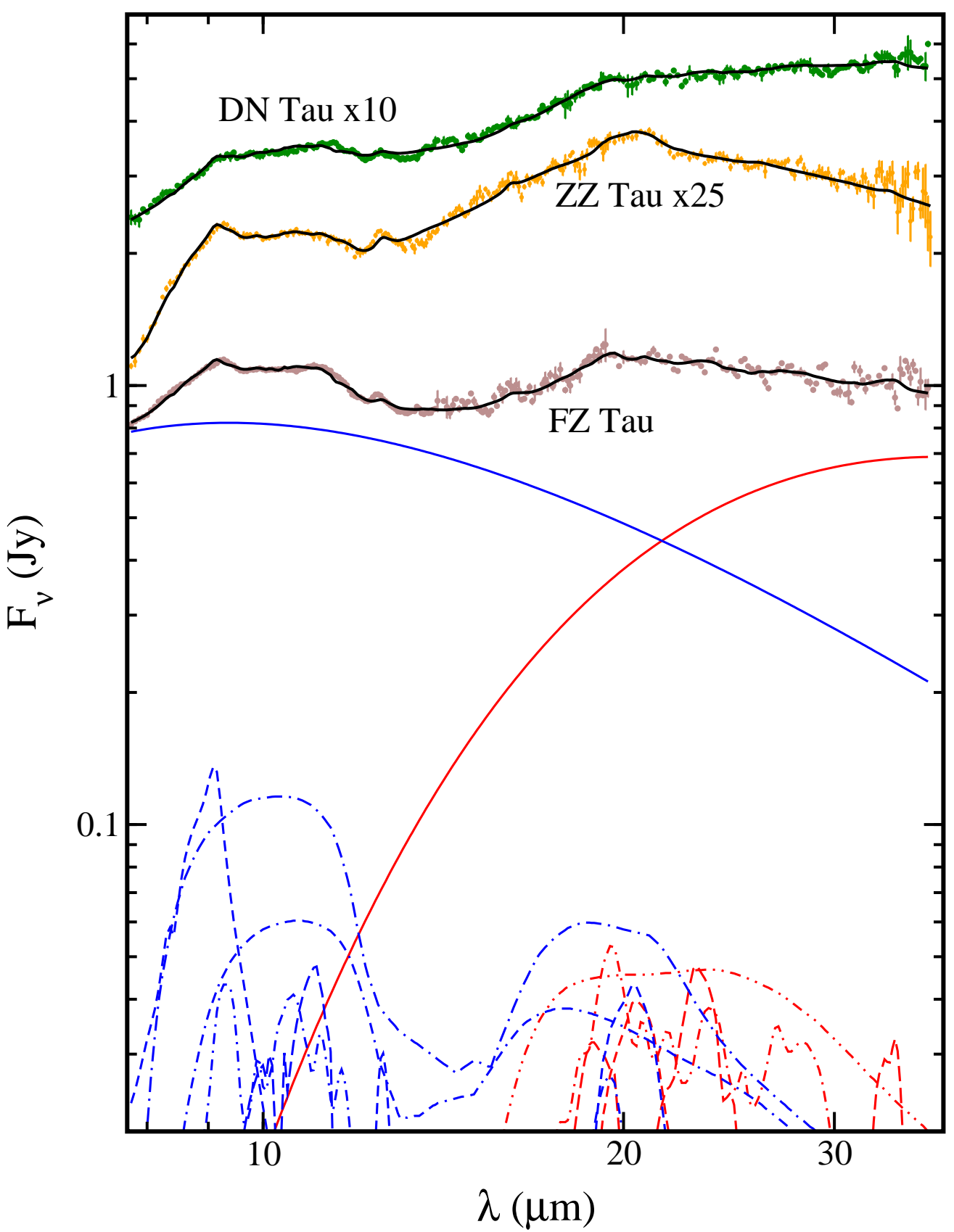

Fig. 7.- Spectra of DN Tau, ZZ Tau, and FZ Tau. DN Tau and FZ Tau require silica but are not fit well by our silica opacity, while ZZ Tau requires silica and is fairly fit well by our silica opacity. Model components are shown for FZ Tau, same style and color convention as Figure 4. 


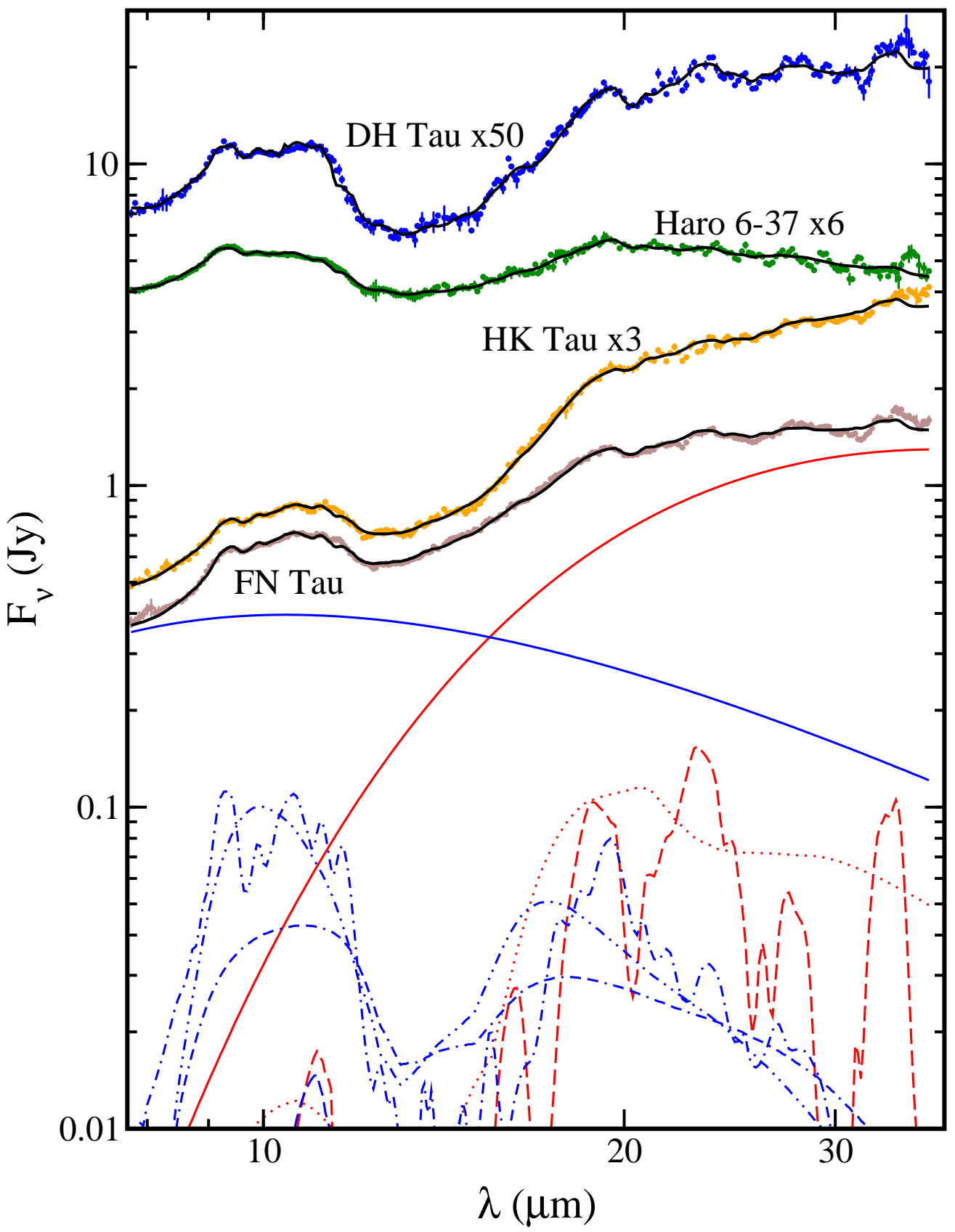

Fig. 8. - Spectra with prominent enstatite features. Model components are shown for FN Tau, same style and color convention as Figure 4. 


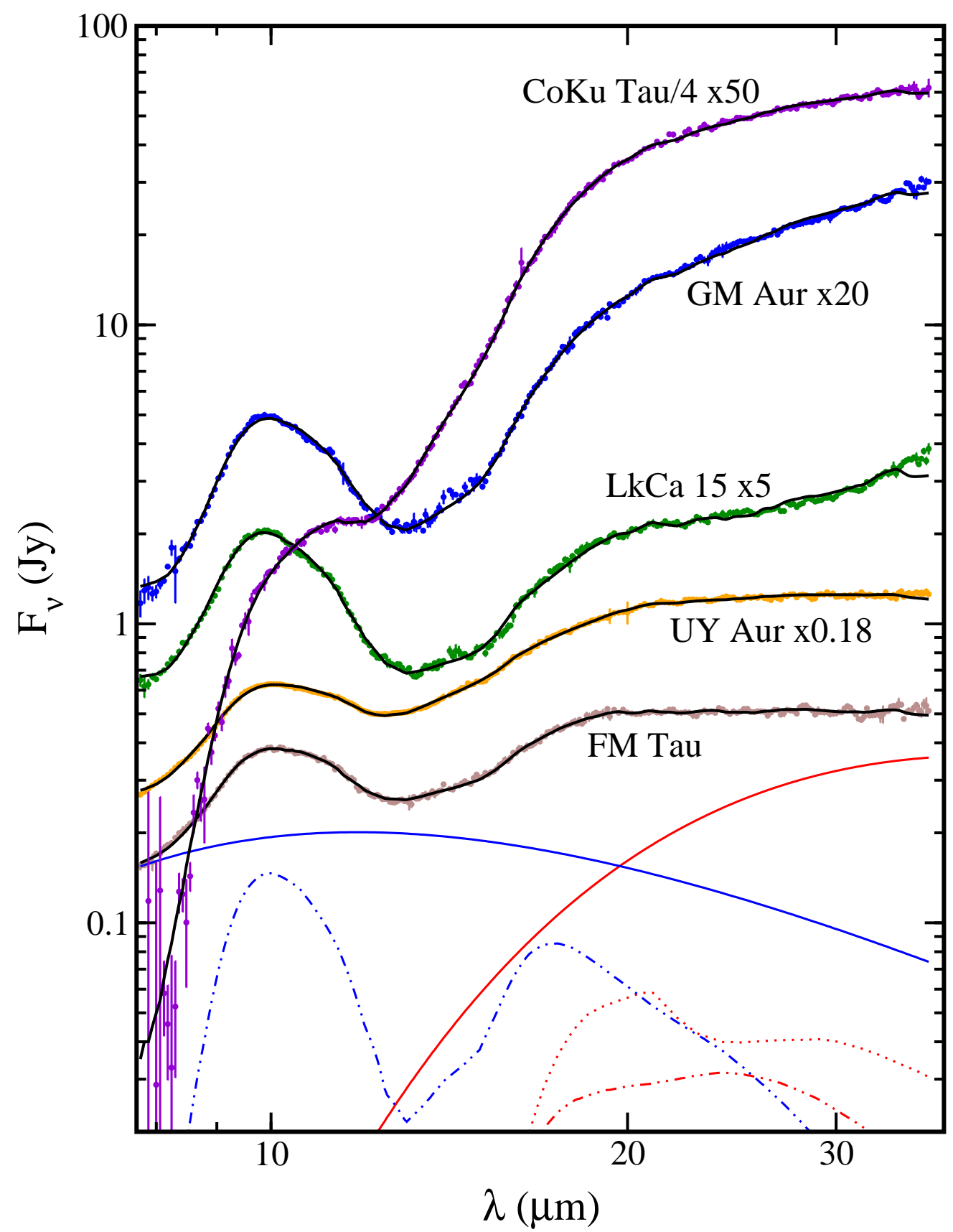

Fig. 9.- Spectra whose models require high abundances of submicron amorphous silicates. Model components are shown for FM Tau, same style and color convention as Figure 4. 


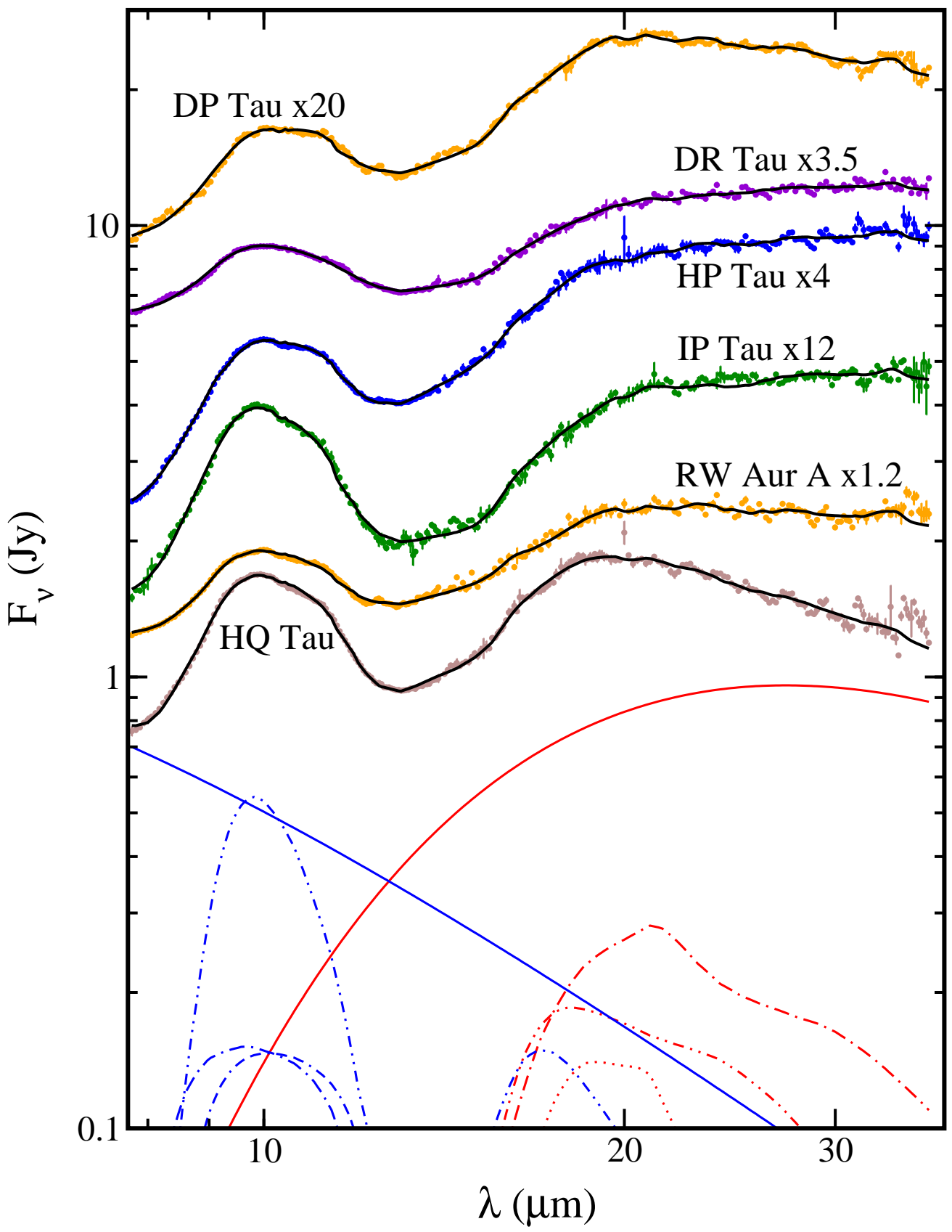

Fig. 10.- Spectra whose models require fairly high abundances of submicron amorphous silicates, though not as high as those in Figure 9. Model components are shown for HQ Tau, same style and color convention as Figure 4. 


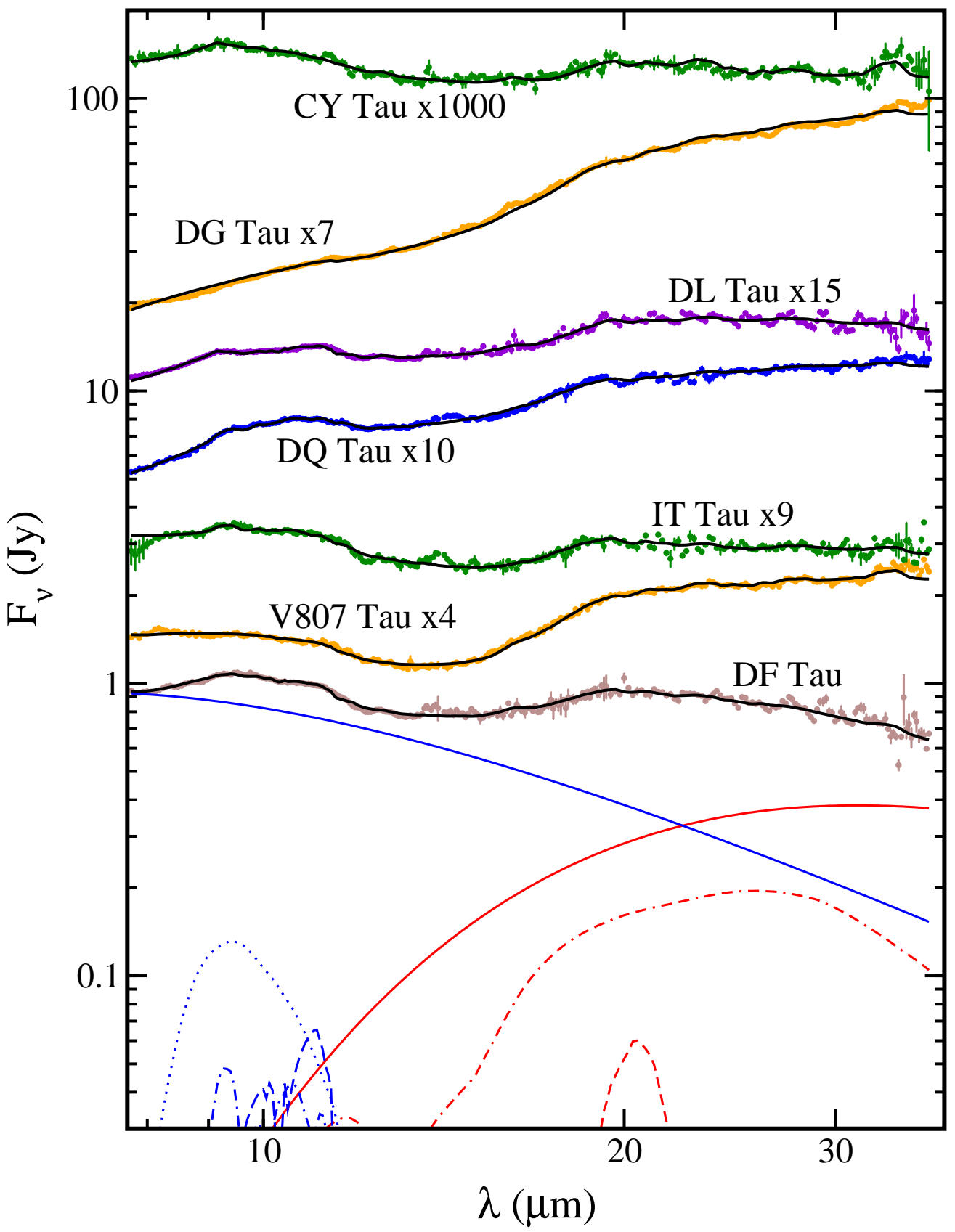

Fig. 11. - Spectra with low equivalent width $10 \mu \mathrm{m}$ complexes. Model components are shown for DF Tau, same style and color convention as Figure 4. 


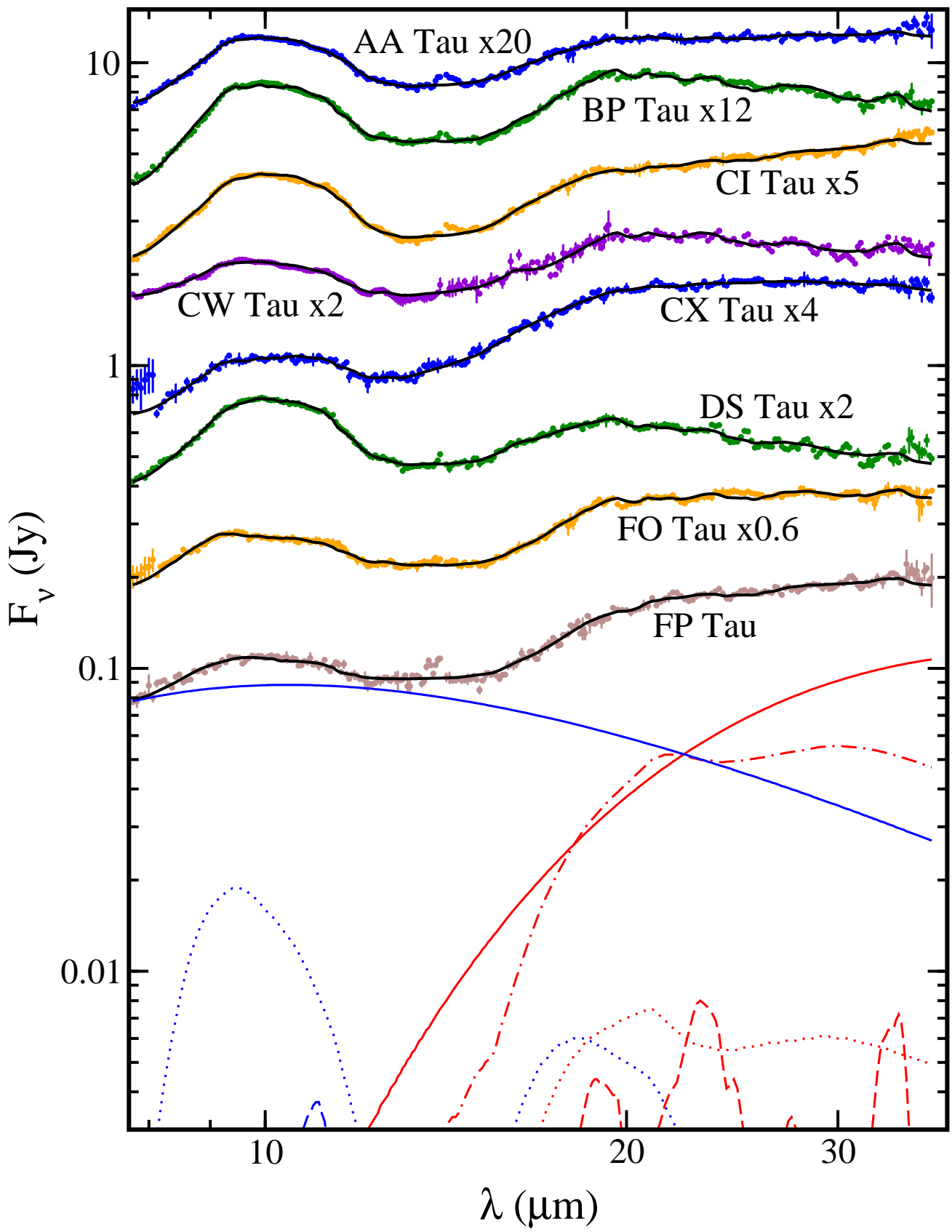

Fig. 12.- Spectra whose models indicate mixed compositions, with no dust component clearly dominant over another. Model components are shown for FP Tau, same style and color convention as Figure 4. 


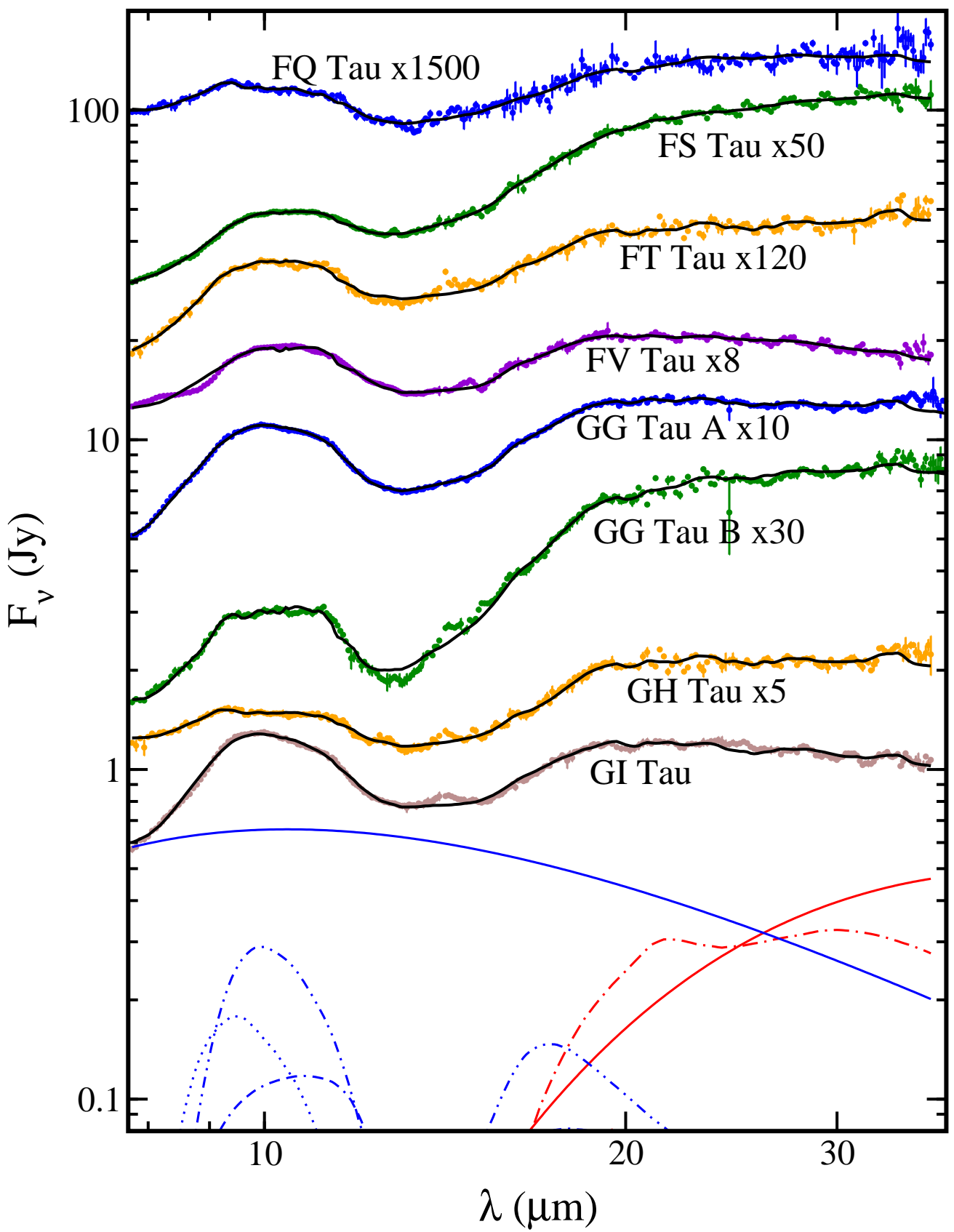

Fig. 13.- Spectra whose models indicate mixed compositions, with no dust component clearly dominant over another. Model components are shown for GI Tau, same style and color convention as Figure 4. 


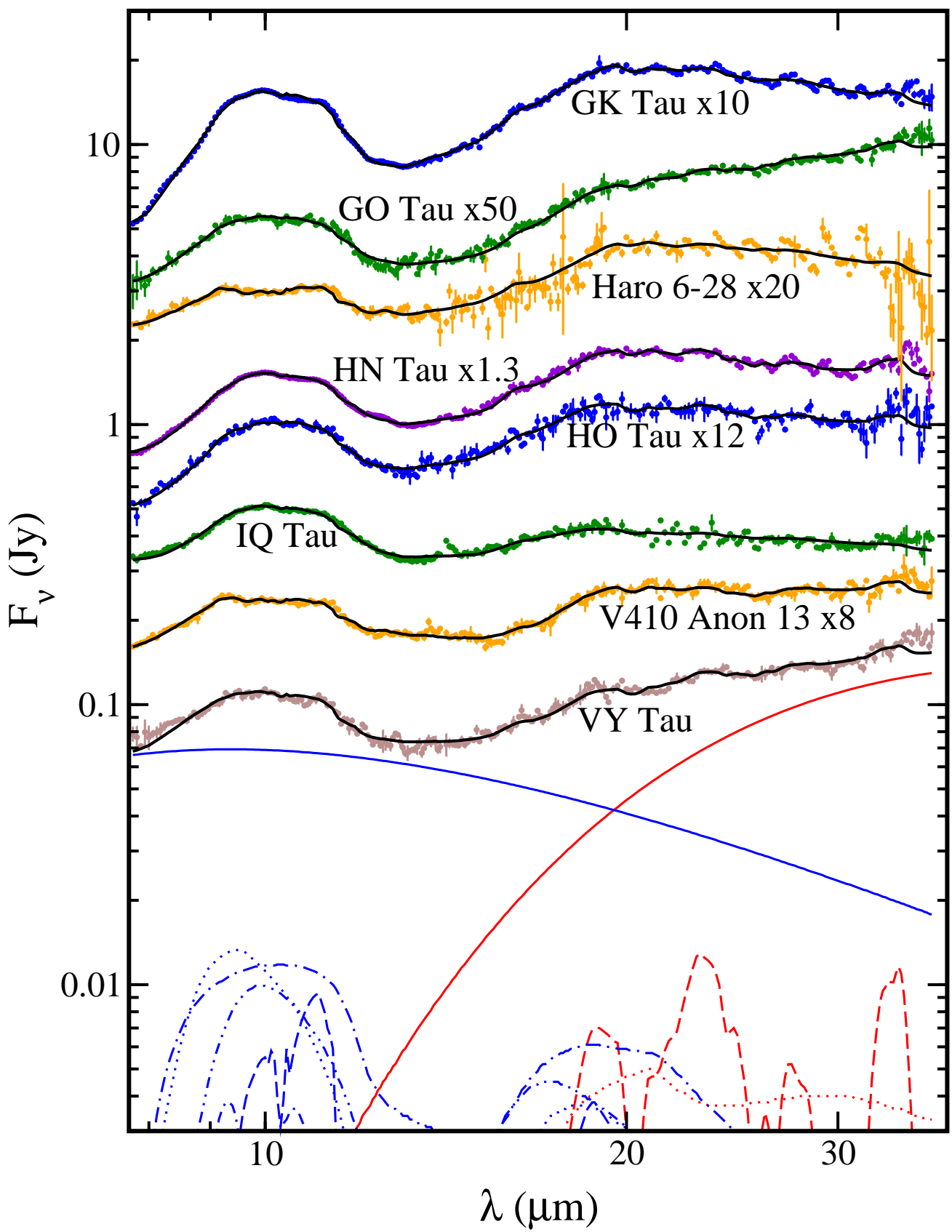

Fig. 14.- Spectra whose models indicate mixed compositions, with no dust component clearly dominant over another. Model components are shown for VY Tau, same style and color convention as Figure 4. 


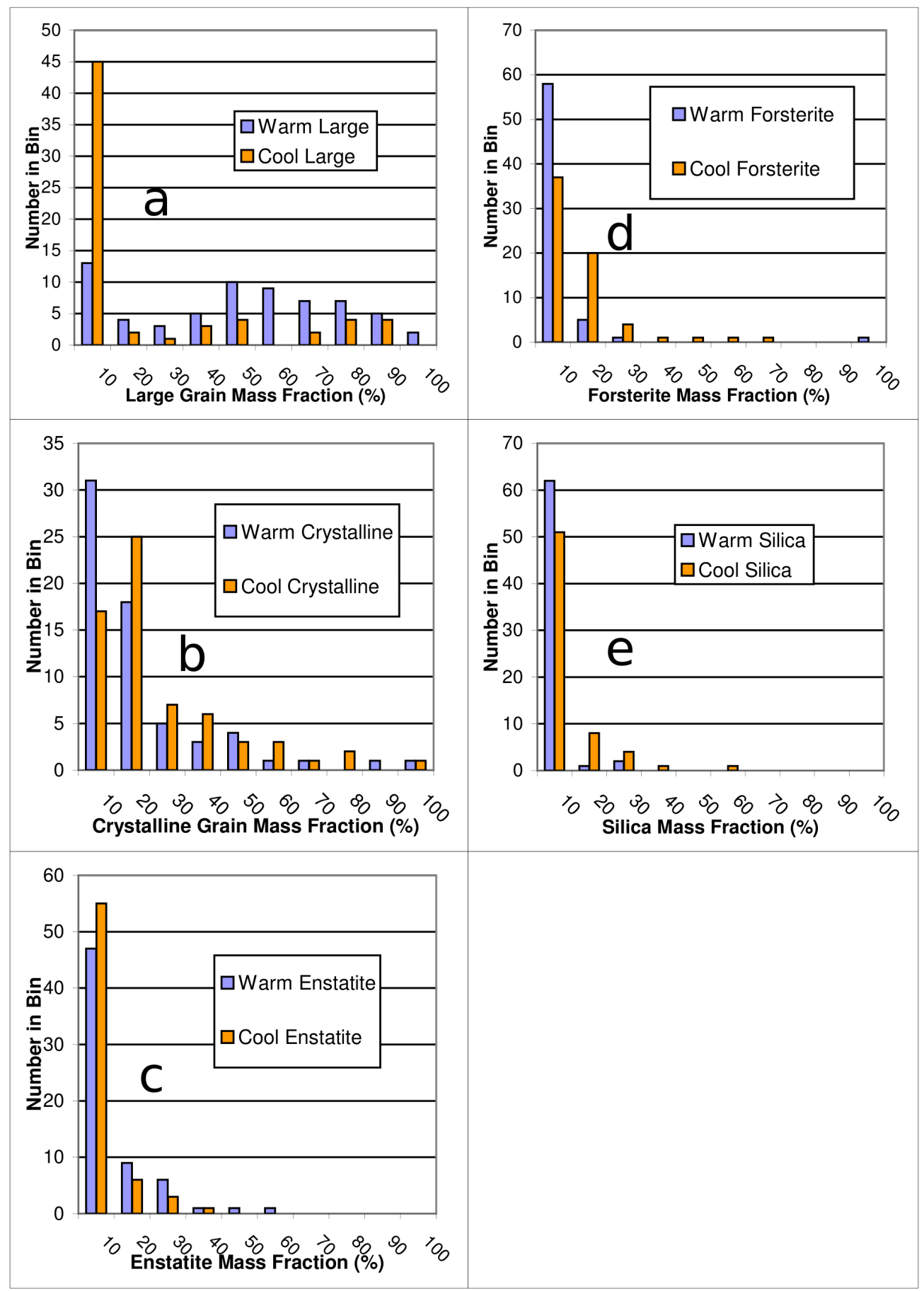

Fig. 15. - Warm versus cool dust histograms. In the upper left is the large grain mass fraction histogram. In the middle left is the crystalline grain mass fraction histogram. In the lower left is the enstatite grain mass fraction histogram. In the upper right is the forsterite grain mass fraction histogram. In the lower right is the silica grain mass fraction histogram. The number of models requiring warm grain mass fractions within a given bin are represented by the height of the blue rectangle to the left within that bin. Orange rectangles to the right are for cool grain mass fractions. 


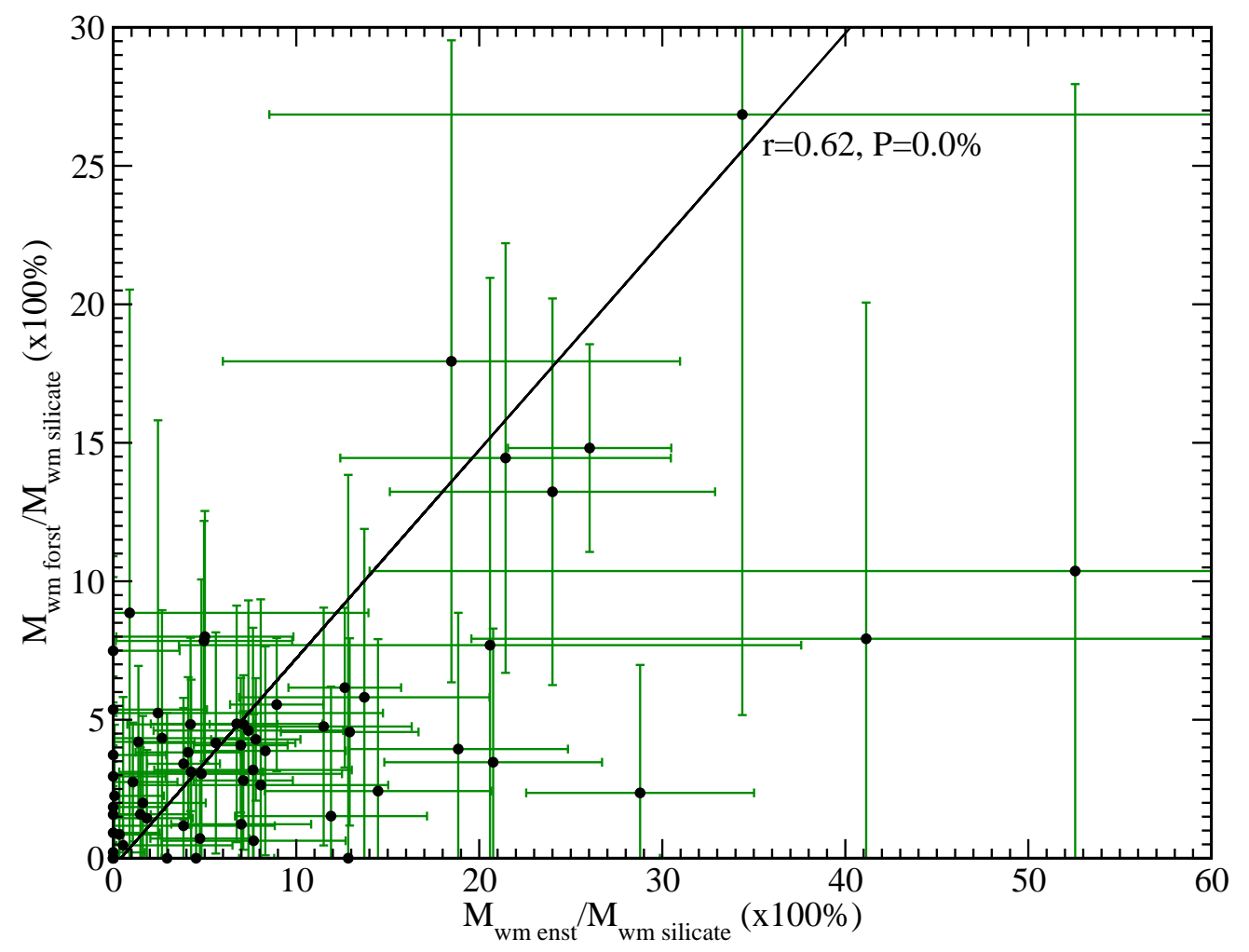

Fig. 16. - Warm forsterite mass fraction versus warm enstatite mass fraction trend plot. The thick solid line running through the points is the trendline consistent with the computed correlation coefficient, indicated on the plot. 


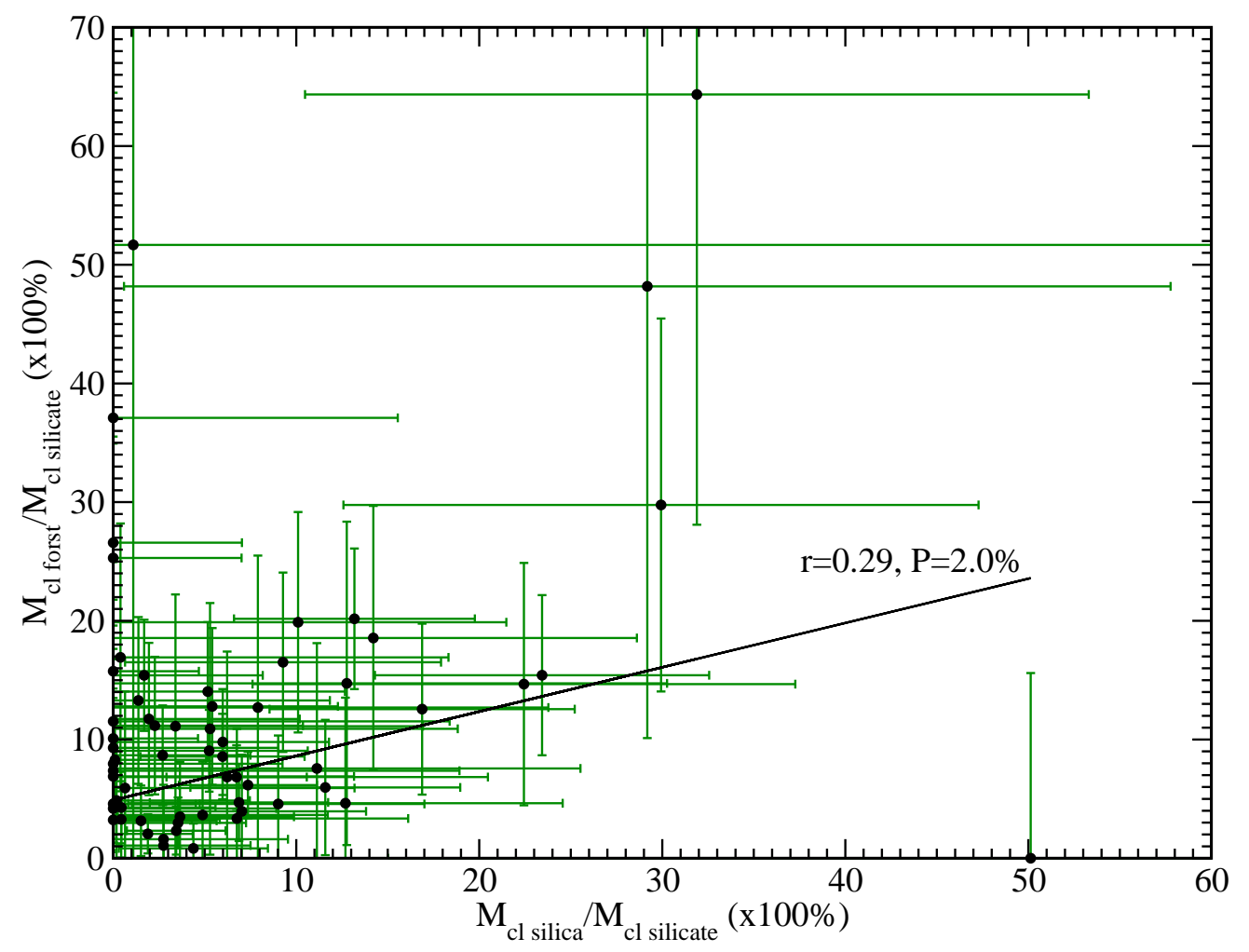

Fig. 17.- Cool forsterite mass fraction versus cool silica mass fraction trend plot. The thick solid line running through the points is the trendline consistent with the computed correlation coefficient, indicated on the plot. 


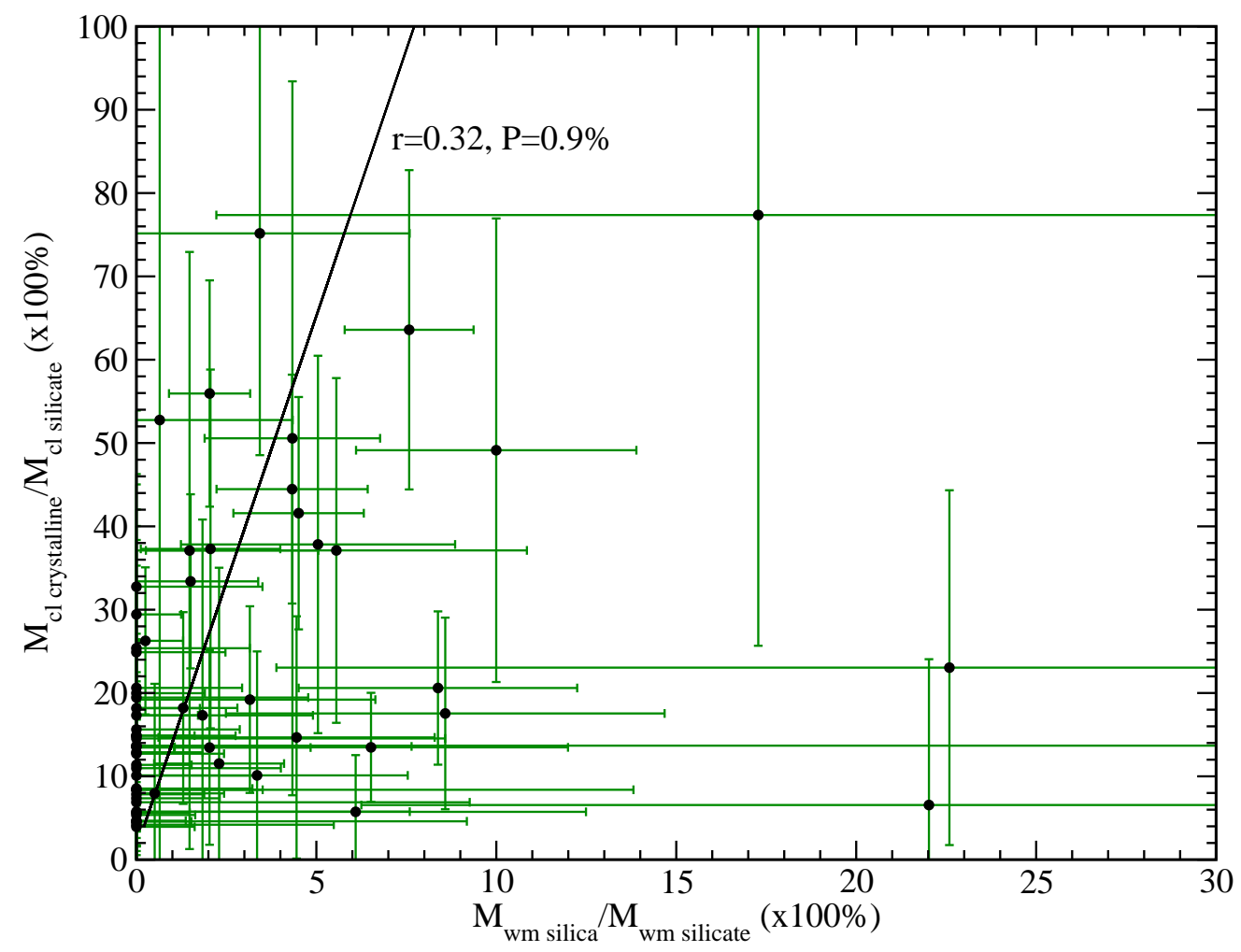

Fig. 18. - Cool crystalline mass fraction versus warm silica mass fraction trend plot. The thick solid line running through the points is the trendline consistent with the computed correlation coefficient, indicated on the plot. 


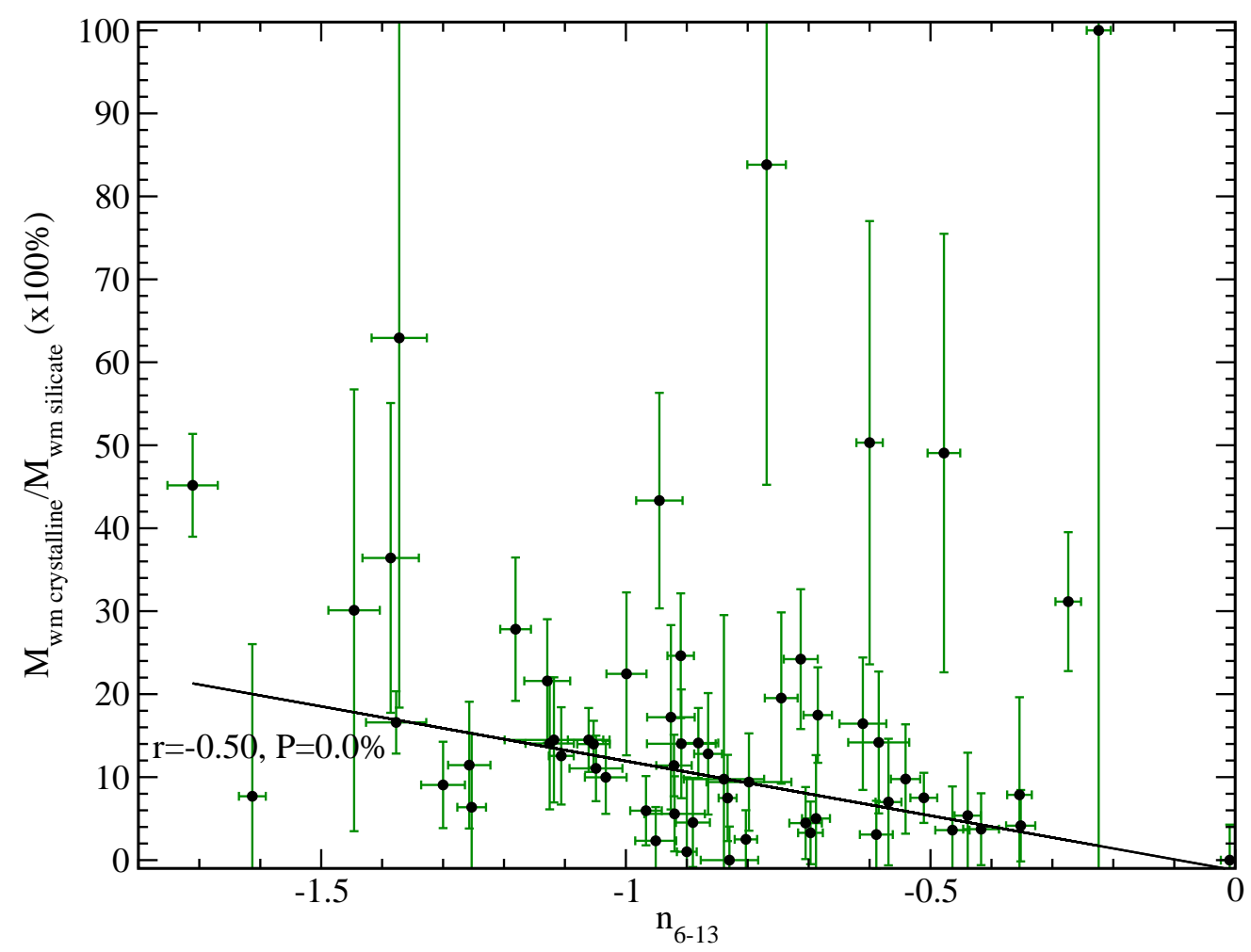

Fig. 19. - Warm crystalline mass fraction versus $\mathrm{n}_{6-13}$ trend plot. The thick solid line running through the points is the trendline consistent with the computed correlation coefficient, indicated on the plot. 


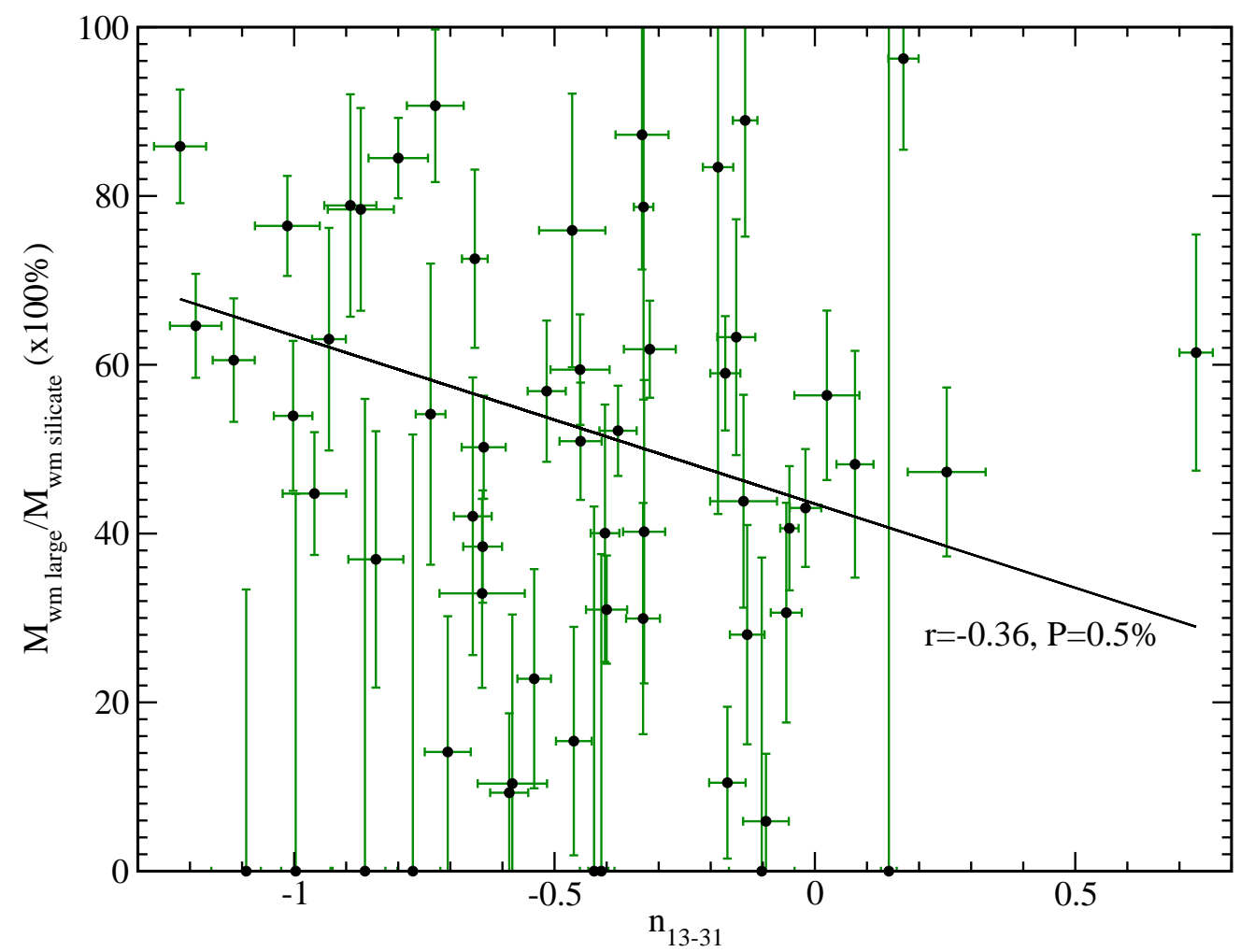

Fig. 20.- Cool crystalline mass fraction versus $n_{13-31}$ trend plot. The thick solid line running through the points is the trendline consistent with the computed correlation coefficient, indicated on the plot. 


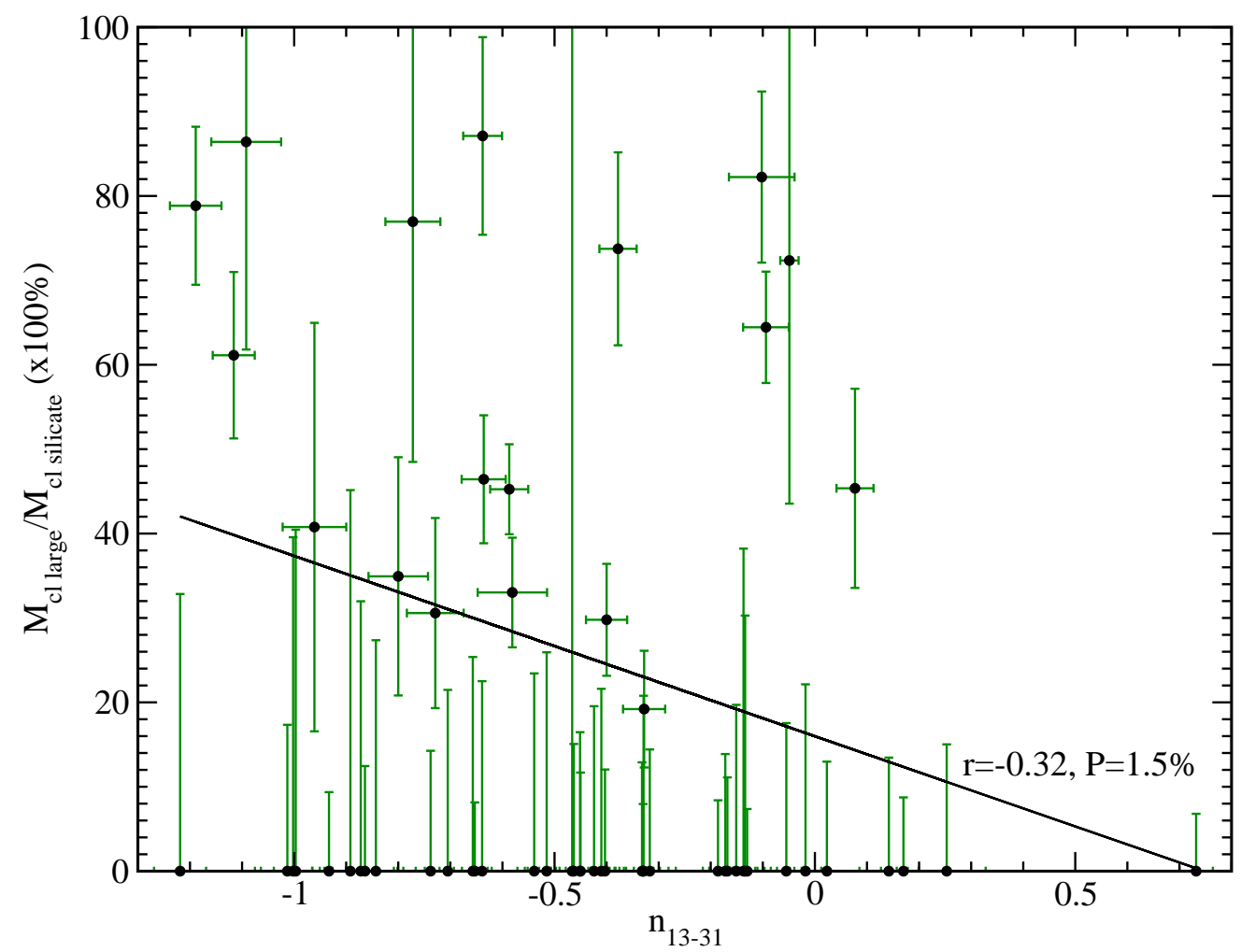

Fig. 21. - Warm large grain mass fraction versus $\mathrm{n}_{13-31}$ trend plot. The thick solid line running through the points is the trendline consistent with the computed correlation coefficient, indicated on the plot. 


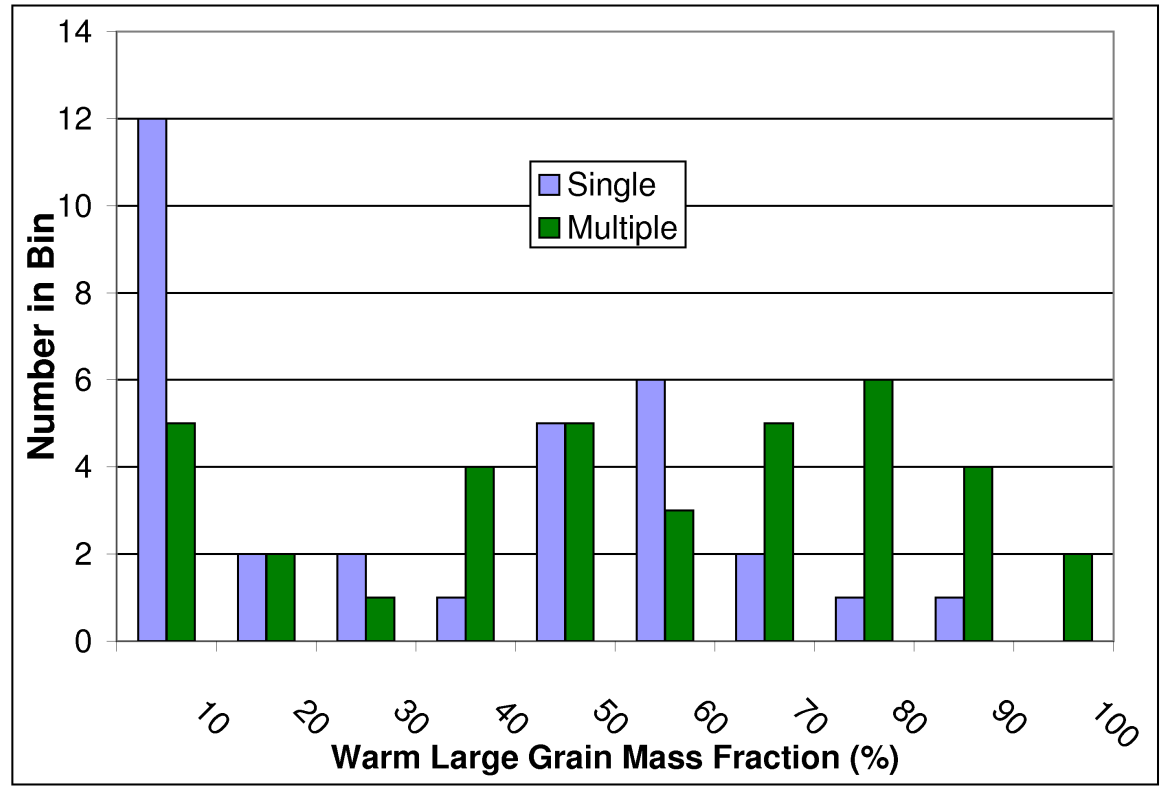

Fig. 22. - Warm large grain mass fraction histograms for single systems (blue) and multiple systems (green). 


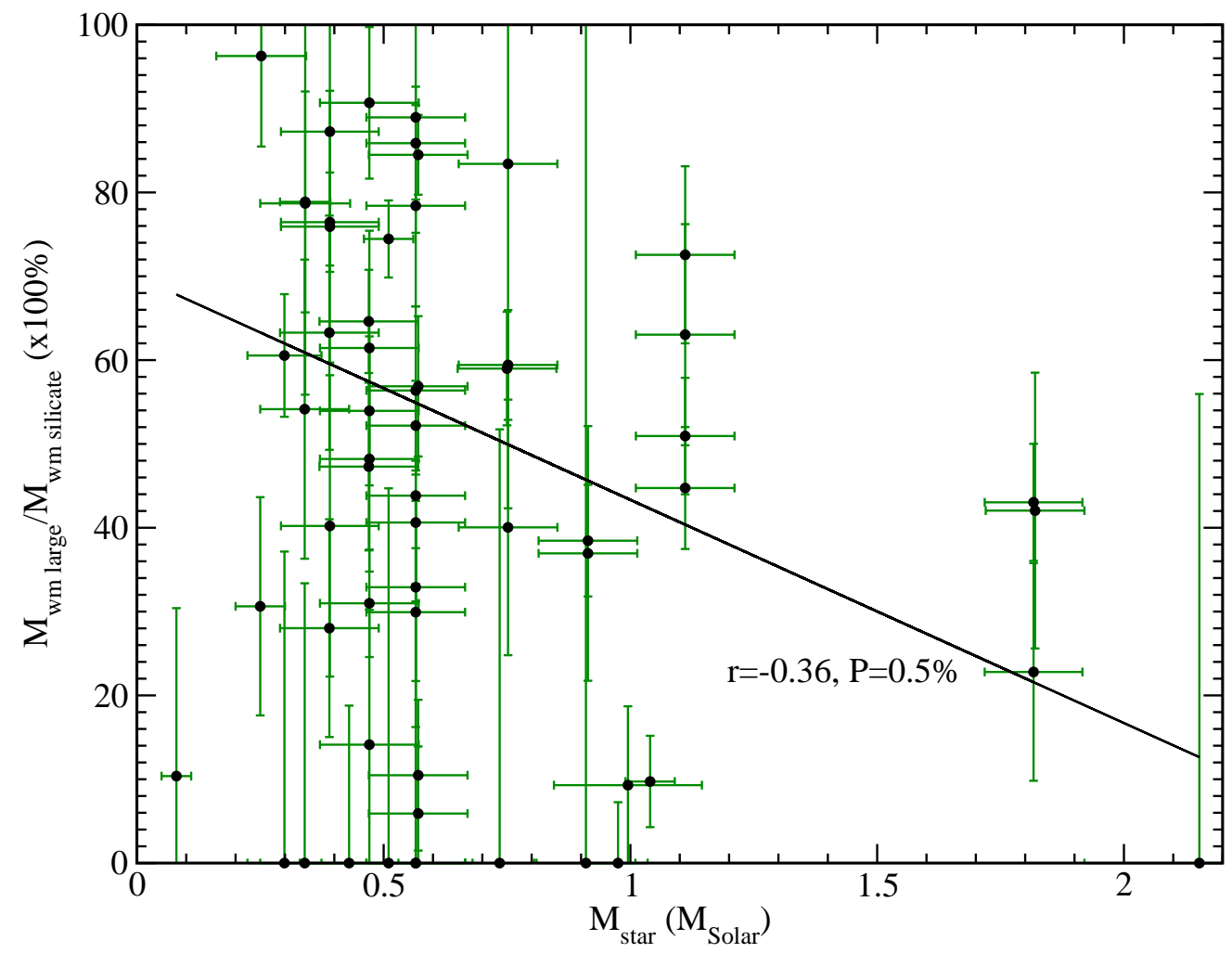

Fig. 23. - Warm large grain mass fraction versus stellar mass trend plot. The thick solid line running through the points is the trendline consistent with the computed correlation coefficient, indicated on the plot. 


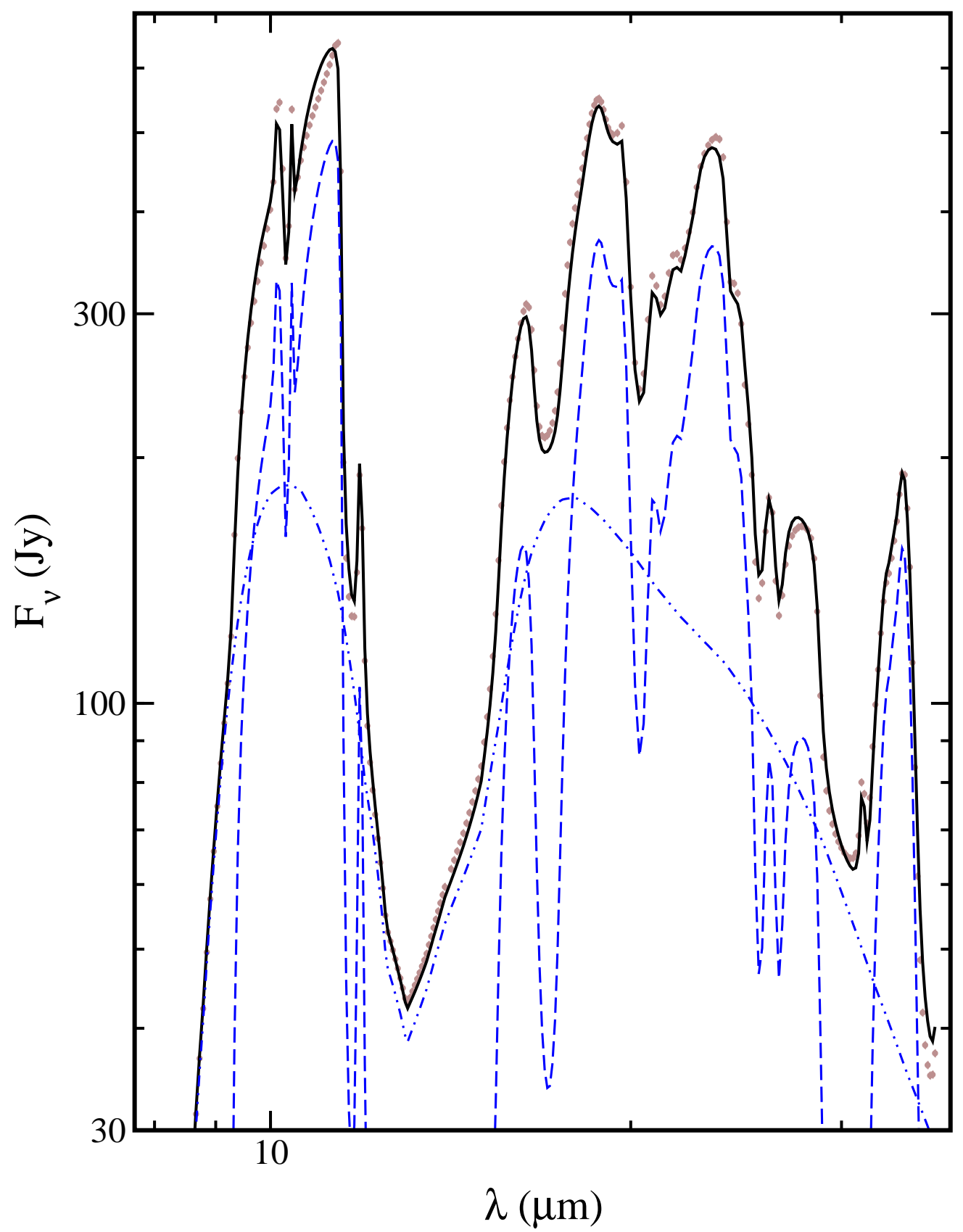

Fig. A1.- Model of Rayleigh-limit size porous aggregate grain composed of amorphous olivine and forsterite. Style and color convention of model components same as for Figure 4. 


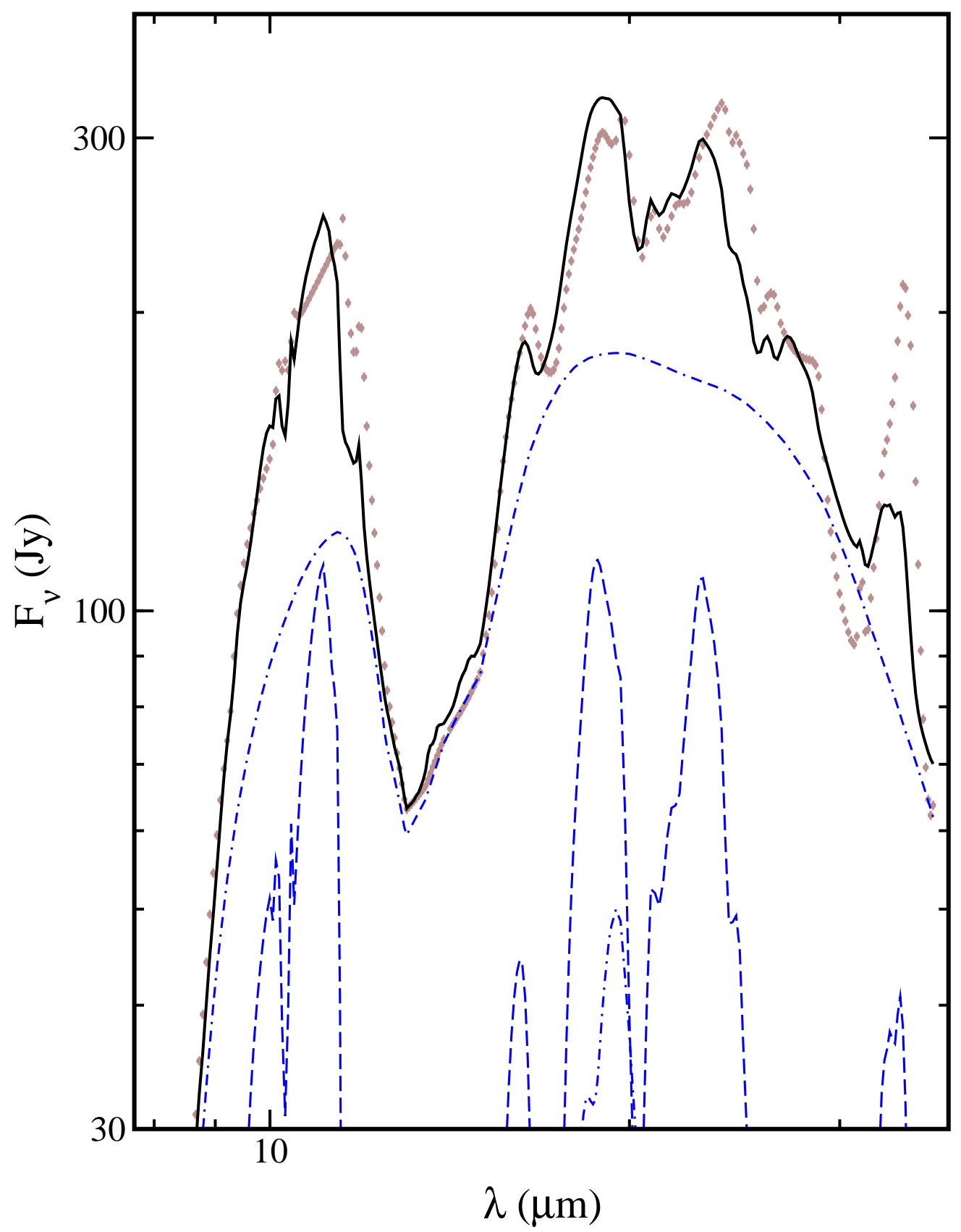

Fig. A2. - Model of $5 \mu \mathrm{m}$ radius porous aggregate grain composed of amorphous olivine and forsterite. Style and color convention of model components same as for Figure 4. 


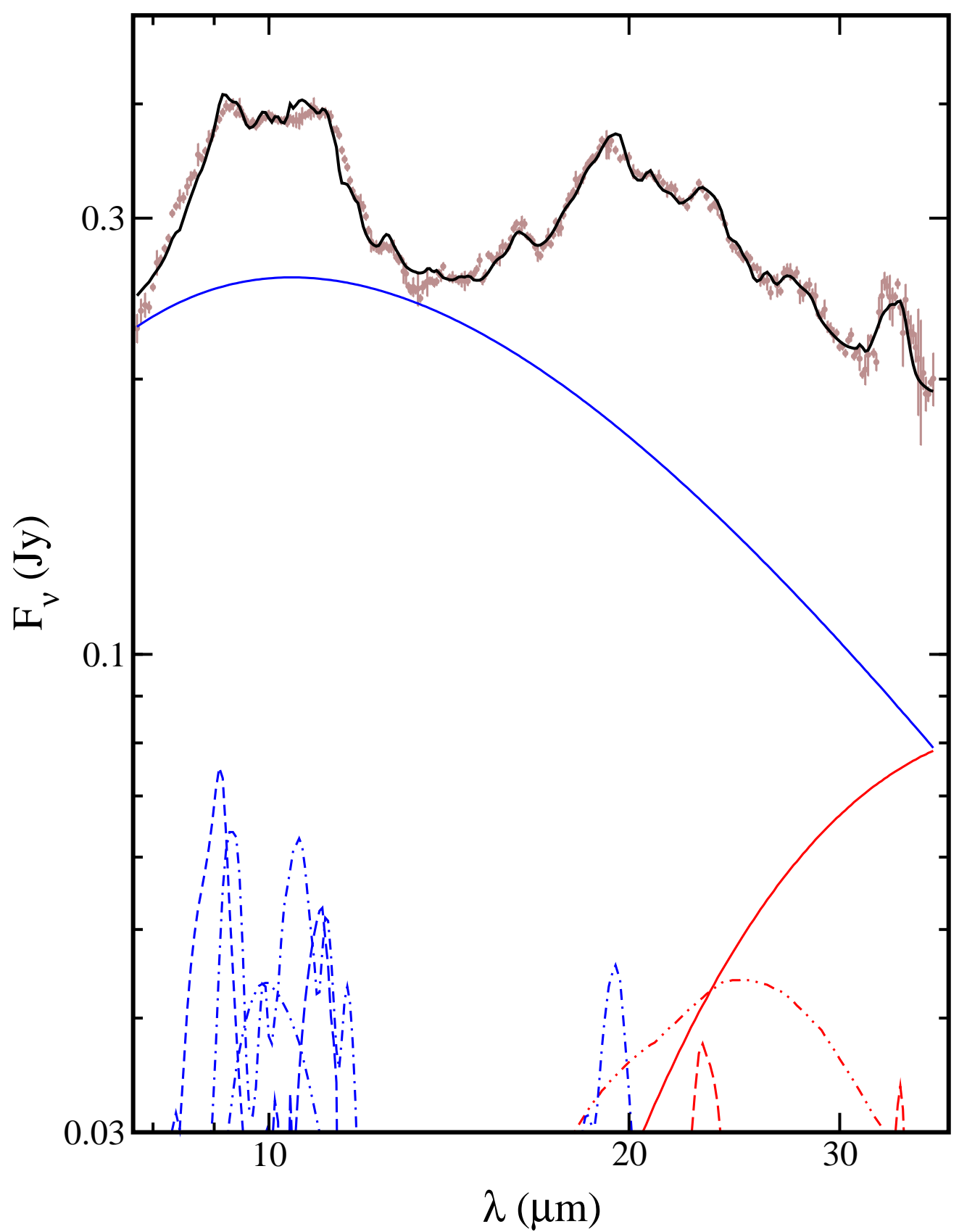

Fig. A3.- Model of IS Tau, without using large grains at either model dust temperature. Reduced $\chi^{2}$ is 4.9, which is 1.6 higher than the 3.3 computed for the model of IS Tau using large grains (see Fig. 5). Style and color convention of model components same as for Figure 4 . 


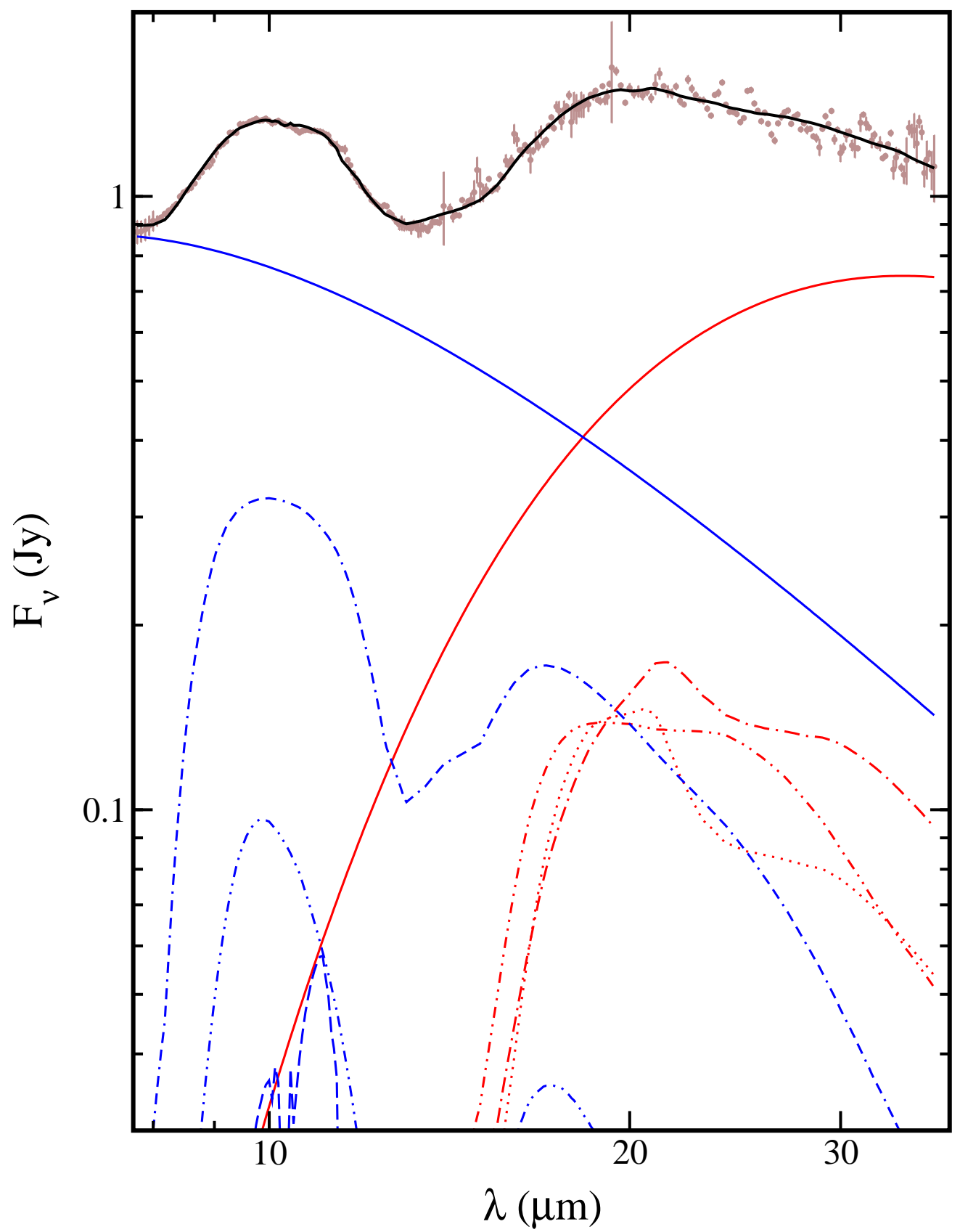

Fig. A4.- Model of UZ Tau/e, replacing $5 \mu \mathrm{m}$ radius $60 \%$ vacuum porous grains of amorphous olivine with $20 \mu \mathrm{m}$ radius $88 \%$ vacuum porous grains of amorphous olivine. Style and color convention of model components same as for Figure 4. 
Table 1: Significance (mass/uncertainty) of Opacities for Forsterite Exemplars

\begin{tabular}{|c|c|c|c|c|c|c|c|c|}
\hline Opacity & DK Tau & GN Tau & $\begin{array}{l}\text { HBC } 656 \\
\end{array}$ & IS Tau & ROXs $42 \mathrm{C}$ & $\begin{array}{l}\text { V836 Tau } \\
\end{array}$ & V955 Tau & \\
\hline $\begin{array}{l}\text { CfoJ98 } \\
\text { (f) }\end{array}$ & 0 & 4.3 & 5.0 & 1.4 & 0.7 & 0 & 0 & $\longleftarrow$ \\
\hline ColJ98 & 4.5 & 0 & 0 & 0 & 0 & 0 & 0 & \\
\hline ChoJ98 & 0 & 0 & 0 & 0 & 0 & 0 & 0.1 & \\
\hline CfaJ98 & 0 & 0 & 0 & 0 & 0.9 & 0 & 0 & \\
\hline CFo91.4 & 0 & 0 & 0 & 0 & 0 & 0 & 0 & \\
\hline CFo89.3 & 0 & 0 & 0 & 0 & 0 & 1.6 & 0 & \\
\hline CFo86.5 & 0 & 0 & 0 & 0 & 0 & 0 & 1.8 & \\
\hline CFo84.4 & 0.4 & 0 & 0 & 0 & 0 & 0 & 0 & \\
\hline CFo80 & 0 & 0 & 0 & 0 & 0 & 0 & 0 & \\
\hline CFo21.8 & 0 & 0 & 0.6 & 0 & 0 & 0 & 0 & \\
\hline Cannfor & 0 & 0 & 0 & 0 & 0 & 0 & 0 & \\
\hline Cfohyb & 0 & 0 & 1.8 & 1.0 & 0 & 0 & 0.3 & $\longleftarrow$ \\
\hline CS6CDE & 0 & 0 & 0.9 & 0.1 & 0 & 0 & 0.8 & $\Longleftarrow$ \\
\hline CS6CDE2 & 1.3 & 0 & 2.6 & 2.7 & 3.2 & 1.1 & 2.2 & $\Longleftarrow$ \\
\hline WfoJ98 & 0 & 0 & 0 & 0 & 0 & 0 & 0 & \\
\hline WolJ98 & 0 & 0 & 0 & 0 & 0 & 0 & 0 & \\
\hline WhoJ98 & 0 & 0 & 0 & 0 & 0.3 & 0 & 0 & \\
\hline WfaJ98 & 3.1 & 0 & 0.9 & 2.5 & 2.4 & 0.4 & 1.7 & $\longleftarrow$ \\
\hline WFo91.4 & 0 & 0 & 0 & 0 & 0 & 0 & 1.2 & \\
\hline WFo 89.3 & 0 & 0 & 0 & 0 & 0 & 0 & 0 & \\
\hline WFo86.5 & 0 & 0 & 0 & 1.3 & 0 & 0 & 0 & \\
\hline WFo 84.4 & 0.1 & 0 & 0 & 0 & 0 & 0 & 0 & \\
\hline WFo80 & 0 & 1.2 & 1.1 & 0 & 0.6 & 0 & 0 & \\
\hline WFo 21.8 & 0 & 0 & 2.1 & 0 & 0 & 0 & 0 & \\
\hline Wannfor & 4.0 & 0 & 2.6 & 1.2 & 0 & 3.3 & 0 & $\longleftarrow$ \\
\hline Wfohyb & 0 & 0 & 0 & 0 & 0 & 0.6 & 0 & \\
\hline WS6CDE & 0 & 0.1 & 0.9 & 0 & 0 & 1.2 & 0 & $\Longleftarrow$ \\
\hline WS6CDE2 & 0.6 & 0.4 & 2.6 & 0.5 & 0.9 & 0 & 0.4 & $\Longleftarrow$ \\
\hline
\end{tabular}

Note. - A given entry in a column is the mass weight of the component named in the first entry on the same row divided by its uncertainty, giving the significance of a particular opacity used in a given model. The naming convention is as described in Section 3.2. Arrows in the final column indicate recurring opacities as discussed in the text. The opacity used most at both temperatures is the CDE2 opacity using Sogawa et al. (2006) optical constants, with the CDE opacity from the same optical constants also frequently included. Both of these are indicated by double-lined arrows. 
Table 2: Significance (mass/uncertainty) of Opacities for Enstatite and Forsterite Exemplars, Jointly

\begin{tabular}{|c|c|c|c|c|c|c|c|c|c|c|c|c|}
\hline Opacity & $\begin{array}{l}\mathrm{DH} \\
\text { Tau }\end{array}$ & $\begin{array}{l}\text { FN } \\
\text { Tau }\end{array}$ & $\begin{array}{c}\text { Haro } \\
6-37\end{array}$ & $\begin{array}{l}\text { HK } \\
\text { Tau }\end{array}$ & $\begin{array}{l}\text { DK } \\
\text { Tau }\end{array}$ & $\begin{array}{l}\text { GN } \\
\text { Tau }\end{array}$ & $\begin{array}{c}\mathrm{HBC} \\
656\end{array}$ & $\begin{array}{c}\text { IS } \\
\text { Tau }\end{array}$ & $\begin{array}{c}\text { ROXs } \\
42 \mathrm{C}\end{array}$ & $\begin{array}{c}\text { V836 } \\
\text { Tau }\end{array}$ & $\begin{array}{c}\text { V955 } \\
\text { Tau }\end{array}$ & \\
\hline Cen90 & 0 & 0 & 1.6 & 0 & 1.9 & 2.1 & 0 & 0 & 0 & 0.9 & 0 & $\Longleftarrow$ \\
\hline Cen 80 & 0 & 0 & 0 & 0 & 0 & 0 & 0 & 0 & 0 & 0 & 0 & \\
\hline Cen70 & 0 & 0 & 0 & 0 & 0 & 0 & 1.0 & 0 & 0 & 0 & 0 & \\
\hline Cen50 & 0 & 0 & 0 & 0 & 0 & 0 & 0.1 & 0 & 0 & 0 & 0 & \\
\hline Cen00 & 0 & 0 & 0 & 0.4 & 0 & 0 & 0 & 1.0 & 2.1 & 0 & 1.4 & \\
\hline CJ98CDE2 & 2.7 & 2.7 & 0 & 0.7 & 0 & 1.5 & 2.5 & 0.7 & 0.9 & 0 & 0 & $\longleftarrow$ \\
\hline CKOso & 0 & 0 & 0 & 0 & 0 & 0 & 0 & 0 & 0 & 0 & 0 & \\
\hline CJ98b & 0 & 0 & 0 & 0 & 1.4 & 0 & 0 & 0 & 0 & 0 & 0 & \\
\hline CJ98c & 0.1 & 0 & 0 & 0 & 0 & 0 & 3.4 & 0 & 2.7 & 0.4 & 0 & \\
\hline CS6CDE2 & 0 & 0 & 0 & 0 & 0 & 0 & 0 & 0 & 2.3 & 0 & 0 & \\
\hline CS6tCDE0.10 & 0 & 0 & 0.9 & 2.6 & 2.2 & 0 & 0 & 4.6 & 0 & 2.4 & 3.6 & $\Longleftarrow$ \\
\hline CS6tCDE0.08 & 0 & 0 & 0 & 0 & 0 & 0 & 0 & 0 & 1.5 & 0 & 0 & \\
\hline CS6tCDE0.06 & 0 & 0 & 0 & 0 & 0 & 2.6 & 0 & 0 & 0 & 0 & 0 & \\
\hline CS6tCDE0.04 & 4.0 & 3.3 & 0 & 0 & 0 & 0 & 8.1 & 0 & 0 & 0 & 0 & \\
\hline Wen90 & 6.2 & 3.5 & 3.6 & 3.6 & 3.5 & 0.2 & 4.9 & 2.1 & 0.3 & 2.5 & 1.3 & $\Longleftarrow$ \\
\hline Wen 80 & 0 & 0 & 0 & 0 & 0 & 0 & 0 & 0 & 0.3 & 0 & 0.5 & \\
\hline Wen70 & 0.7 & 0 & 0 & 0 & 0 & 0 & 0 & 0 & 0 & 0 & 0 & \\
\hline Wen50 & 0 & 0 & 0 & 0 & 0 & 0 & 0 & 0 & 0 & 0 & 0 & \\
\hline Wen00 & 1.8 & 0 & 2.6 & 1.9 & 2.4 & 0 & 3.2 & 1.0 & 0 & 4.3 & 0 & $\longleftarrow$ \\
\hline WJ98CDE2 & 0 & 0 & 0.3 & 0.2 & 0 & 0 & 0.5 & 0 & 0 & 0 & 0 & \\
\hline WKOso & 0 & 2.0 & 0 & 0 & 0 & 0 & 0 & 0 & 0 & 0 & 0 & \\
\hline WJ98b & 0 & 0 & 0 & 0 & 0 & 0 & 0 & 0 & 0 & 0 & 0 & \\
\hline WJ98c & 0 & 0 & 0 & 0 & 0 & 0 & 0 & 0 & 0 & 0 & 0 & \\
\hline WS6CDE2 & 0 & 0 & 0 & 0 & 0 & 0 & 0 & 0 & 0 & 0 & 0 & \\
\hline WS6tCDE0.10 & 4.2 & 0.9 & 1.2 & 0 & 2.9 & 0 & 3.2 & 1.4 & 0 & 2.0 & 0 & $\Longleftarrow$ \\
\hline WS6tCDE0.08 & 0 & 0 & 0 & 0 & 0 & 0 & 2.4 & 0 & 1.3 & 0 & 0 & \\
\hline WS6tCDE0.06 & 0 & 0 & 0 & 0 & 0 & 0 & 0 & 0 & 0 & 0 & 1.2 & \\
\hline WS6tCDE0.04 & 0 & 0 & 0 & 0.9 & 0 & 1.3 & 0 & 0 & 0 & 0 & 0 & \\
\hline
\end{tabular}

Note. - Entries in the table are mass weight of a component divided by uncertainty, as in Table 1. Arrows in the final column indicate recurring opacities as discussed in the text. The enstatite opacity used most at both temperatures is the "en90" opacity from Chihara et al. (2002), indicated by double-lined arrows. The optimal forsterite opacity is the tCDE opacity using optical constants by Sogawa et al. (2006) with bounding parameter $\mathrm{t}=0.10$, indicated by double-lined arrows . 
Table 3: Significance (mass/uncertainty) of Opacities for Amorphous Silicate Exemplars

\begin{tabular}{|c|c|c|c|c|c|c|c|c|}
\hline Opacity & "CoKu Tau/4 & "DM Tau & "FM Tau & "GM Aur & $\overline{\mathrm{LkCa} 15}$ & TW Cha & UY Aur & \\
\hline CD95OlSm & 0 & 0 & 0 & 0 & 0 & 0 & 0 & $\Longleftarrow$ \\
\hline CD95OlLg & 0 & 0 & 0 & 0 & 0 & 0 & 0 & $\Longleftarrow$ \\
\hline CD79O1Sm & 5.5 & 0 & 0 & 0 & 0 & 0 & 0 & \\
\hline CD79OlLg & 0 & 0 & 0 & 0 & 0 & 0 & 0 & \\
\hline CD95Py5Sm & 12.4 & 0 & 0 & 0 & 0 & 0 & 1.4 & \\
\hline CD95Py5Lg & 0 & 0 & 0 & 0 & 0 & 0 & 0 & \\
\hline CD95Py10Sm & 6.6 & 0 & 7.3 & 14.1 & 0 & 0 & 0 & $\longleftarrow$ \\
\hline CD95Py10Lg & 0 & 0 & 0 & 0 & 0 & 0 & 0 & \\
\hline CJ94PySm & 0 & 0 & 0 & 4.2 & 12.0 & 10.6 & 0 & $\Longleftarrow$ \\
\hline CJ94PyLg & 0 & 0 & 0 & 0 & 0 & 0 & 0 & $\Longleftarrow$ \\
\hline CD79PySm & 0.8 & 3.0 & 0 & 7.5 & 0 & 0.4 & 5.4 & $\longleftarrow$ \\
\hline CD79PyLg & 0 & 0 & 0 & 0 & 0 & 0 & 0 & \\
\hline CSD96PySm & 0 & 0 & 0 & 0 & 0 & 7.0 & 0 & \\
\hline CSD96PyLg & 0 & 0 & 0 & 0 & 0 & 0 & 0 & \\
\hline WD95OlSm & 8.9 & 5.8 & 15.0 & 16.2 & 23.2 & 22.4 & 9.3 & $\Longleftarrow$ \\
\hline WD95OlLg & 0 & 0 & 0 & 0 & 0 & 0 & 0 & $\Longleftarrow$ \\
\hline WD79OlSm & 0 & 4.3 & 0 & 0.4 & 1.1 & 0 & 1.7 & $\longleftarrow$ \\
\hline WD79OlLg & 0 & 1.0 & 0 & 0 & 0 & 2.5 & 0.1 & $\longleftarrow$ \\
\hline WD95Py5Sm & 0 & 3.0 & 0 & 0 & 0 & 0 & 0 & \\
\hline WD95Py5Lg & 0 & 15.1 & 0.3 & 0 & 0 & 0 & 0 & \\
\hline WD95Py10Sm & 0 & 0.5 & 0.5 & 0 & 0 & 0 & 2.0 & $\longleftarrow$ \\
\hline WD95Py10Lg & 0 & 0 & 3.2 & 0 & 0 & 0 & 0 & $\longleftarrow$ \\
\hline WJ94PySm & 0 & 0 & 0 & 0 & 0 & 0 & 0 & $\Longleftarrow$ \\
\hline WJ94PyLg & 0 & 0 & 0 & 0 & 2.5 & 4.7 & 0 & $\Longleftarrow$ \\
\hline WD79PySm & 0 & 0 & 0 & 0 & 0 & 0 & 0 & \\
\hline WD79PyLg & 0 & 1.7 & 1.1 & 0 & 0 & 0 & 1.8 & $\longleftarrow$ \\
\hline WSD96PySm & 0 & 0 & 0 & 7.7 & 3.5 & 0 & 1.8 & $\longleftarrow$ \\
\hline WSD96PyLg & 0 & 0 & 0 & 0 & 0 & 0 & 0 & \\
\hline
\end{tabular}

Note. - Entries in the table are mass weight of a component divided by uncertainty, as in Table 1. Arrows in the final column indicate recurring opacities as discussed in the text. Of the amorphous olivines, the one included in the most models at the highest significance is the amorphous olivine from Dorschner et al. (1995), indicated by double-lined arrows. Of the amorphous pyroxenes, the one that shows up in the most models at the highest significance is the amorphous pyroxene of cosmic composition by Jaeger et al. (1994), indicated by double-lined arrows. 
Table 4: Trapezium Dust Model Parameters

\begin{tabular}{lcccccc}
\hline \hline Dust & $281 \mathrm{~K}$ & \multicolumn{5}{c}{$145 \mathrm{~K}$} \\
Species & Value & Unc. & Sgnfcnc. & Value & Unc. & Sgnfcnc. \\
$(1)$ & $(2)$ & $(3)$ & $(4)$ & $(5)$ & $(6)$ & $(7)$ \\
\hline Blackbody & 59.6 & 8.8 & 6.8 & $\ldots$ & $\ldots$ & $\ldots$ \\
Sm. Am. Py. & 1.90 & 0.63 & 3.0 & $\ldots$ & $\ldots$ & $\ldots$ \\
Sm. Am. Ol. & 2.05 & 0.75 & 2.7 & 358 & 47 & 7.6 \\
Silica & 0.367 & 0.292 & 1.3 & 23.0 & 29.9 & 0.8 \\
\hline
\end{tabular}

Note. - Col. (1): Dust component. "Sm. Am. Py." and "Sm Am. Ol." denote small amorphous pyroxene and small amorphous olivine, respectively. Cols. (2) and (5): Parameter value for warm and cool dust, respectively. Cols. (3) and (6): Uncertainties on parameter values for warm and cool dust, respectively. Cols. (4) and (7): Significances of component, being the parameter value divided by its uncertainty, for warm and cool dust, respectively. Parameter values and uncertainties for blackbody are in units of $10^{-14}$ steradians, while parameter values and uncertainties for other dust components are in units of $10^{-16} \frac{\mathrm{gm}}{\mathrm{cm}^{2}}$. "..." indicates the component at the temperature indicated by placement in the columns was not included in the model, so its mass is zero. Other components not included in the model (also having zero mass) are large amorphous pyroxene, large amorphous olivine, enstatite, and forsterite. $\chi^{2}$ per degree of freedom for this model is 1.6. 
Table 5: Degeneracies between Dust Components

\begin{tabular}{ccc}
\hline \hline & & Correl. \\
Component & Component & $\begin{array}{c}\text { Coeff. } \\
(1)\end{array}$ \\
\hline Wm. Sm. Am. Py. & Wm. Sm. Am. Ol. & -0.73 \\
Wm. Lg. Am. Py. & Wm. Lg. Am. Ol. & -0.72 \\
Wm. Enstatite & Wm. Silica & -0.50 \\
Cl. Sm. Am. Py. & Cl. Sm. Am. Ol. & $-0.82>\mathrm{r}>-0.89$ \\
Cl. Lg. Am. Py. & Cl. Lg. Am. Ol. & $-0.87 \geq \mathrm{r} \geq-0.89$ \\
Cl. Enstatite & Cl. Forsterite & -0.46 \\
Cl. Enstatite & Cl. Silica & $-0.29 \geq \mathrm{r} \geq-0.50$ \\
\hline
\end{tabular}

Note. - Cols. (1) and (2): pair of dust components with significant degeneracy. "Wm." denotes warm, "Cl." denotes cool, "Sm." denotes small, "Lg." denotes large, "Am." denotes amorphous, "Py." denotes pyroxene, and "Ol." denotes olivine. Col. (3): Correlation coefficients between pair of dust components obtained from covariance matrices. The probability, $\mathrm{P}$, of the correlation coefficient between each of the seven pairs listed in the table being drawn from a random distribution of data is less than or equal to $0.1 \%$, assuming there are 133 independent data points in each spectrum for which the a covariance matrix is computed. 
Table 6. Dust Model Parameters

\begin{tabular}{|c|c|c|c|c|c|c|c|c|c|c|c|c|}
\hline $\begin{array}{l}\text { Object } \\
(1)\end{array}$ & $\begin{array}{c}\text { Temp } \\
(\mathrm{K}) \\
(2)\end{array}$ & $\begin{array}{c}\Omega_{B B} \\
(3)\end{array}$ & $\begin{array}{c}\text { small } \\
\text { Amorphous } \\
\text { Pyroxene }^{\mathrm{a}} \\
(4)\end{array}$ & $\begin{array}{c}\text { small } \\
\text { Amorph. } \\
\text { Olivine }^{\mathrm{b}} \\
(5)\end{array}$ & $\begin{array}{c}\text { large } \\
\text { Amorph. } \\
\text { Pyroxene }^{\mathrm{c}} \\
(6)\end{array}$ & $\begin{array}{c}\text { large } \\
\text { Amorph. } \\
\text { Olivine }^{\mathrm{d}} \\
(7)\end{array}$ & $\begin{array}{c}\text { Crystalline } \\
\text { Enstatite } \\
\text { (8) }\end{array}$ & $\begin{array}{l}\text { Crystalline } \\
\text { Forsterite }^{\mathrm{f}} \\
\quad(9)\end{array}$ & $\begin{array}{c}\text { Crystalline } \\
\text { Silica }^{\mathrm{g}} \\
(10)\end{array}$ & $\begin{array}{l}\text { Total } \\
\text { Dust } \\
\text { Mass } \\
(11)\end{array}$ & $\frac{\chi^{2}}{\text { d.o.f. }}$ & \\
\hline \multirow{2}{*}{$\begin{array}{l}\text { AA Tau } \\
\text { ""? }\end{array}$} & 127 & $123.8 \pm 3.9$ & $75.1 \pm 30.1$ & $0.0 \pm 18.8$ & $0.0 \pm 21.6$ & $0.0 \pm 14.3$ & $6.2 \pm 11.7$ & $7.6 \pm 10.6$ & $11.1 \pm 14.4$ & 59.1 & 2.9 & \\
\hline & 545 & $1.22 \pm 0.02$ & $9.7 \pm 4.4$ & $28.9 \pm 5.0$ & $34.2 \pm 6.1$ & $22.6 \pm 5.8$ & $4.5 \pm 3.5$ & $0.0 \pm 3.2$ & $0.0 \pm 2.5$ & 0.937 & $\cdots$ & \\
\hline \multirow{2}{*}{$\begin{array}{l}\text { BP Tau } \\
\text { “"” }\end{array}$} & 103 & $64.3 \pm 10.9$ & $15.5 \pm 4.9$ & $24.4 \pm 3.8$ & $45.2 \pm 4.6$ & $0.0 \pm 2.6$ & $8.4 \pm 2.5$ & $3.0 \pm 2.1$ & $3.5 \pm 3.7$ & 981 & 3.6 & \\
\hline & 431 & $2.73 \pm 0.05$ & $45.6 \pm 6.4$ & $27.7 \pm 5.2$ & $9.3 \pm 6.9$ & $0.0 \pm 6.4$ & $12.9 \pm 3.8$ & $4.6 \pm 3.4$ & $0.0 \pm 2.8$ & 1.95 & $\ldots$ & \\
\hline \multirow{2}{*}{$\begin{array}{l}\text { CI Tau } \\
\text { "'” }\end{array}$} & 115 & $312.4 \pm 9.93272$ & $80.2 \pm 17.9$ & $11.8 \pm 10.0$ & $0.0 \pm 11.6$ & $0.0 \pm 7.6$ & $0.0 \pm 6.9$ & $8.0 \pm 6.3$ & $0.0 \pm 9.2$ & 278 & 3.3 & \\
\hline & 545 & $1.50 \pm 0.03$ & $0.0 \pm 3.5$ & $35.1 \pm 4.1$ & $47.6 \pm 4.9$ & $11.4 \pm 4.6$ & $4.7 \pm 2.7$ & $0.7 \pm 2.5$ & $0.5 \pm 1.9$ & 1.66 & $\ldots$ & \\
\hline \multirow{2}{*}{$\begin{array}{l}\text { CoKu Tau/3 } \\
\text { ""” }\end{array}$} & 103 & $0.0 \pm 0.0$ & $0.0 \pm 7.8$ & $3.0 \pm 5.6$ & $24.9 \pm 5.5$ & $54.0 \pm 7.6$ & $3.9 \pm 3.6$ & $9.1 \pm 3.4$ & $5.2 \pm 5.4$ & 488 & 4.7 & \\
\hline & 602 & $0.975 \pm 0.019$ & $11.1 \pm 3.2$ & $13.2 \pm 3.4$ & $28.0 \pm 4.4$ & $36.6 \pm 4.3$ & $7.0 \pm 2.6$ & $4.1 \pm 2.4$ & $0.0 \pm 1.8$ & 1.06 & & \\
\hline \multirow{2}{*}{$\begin{array}{l}\text { CoKu Tau/4 } \\
\text { "'”" }\end{array}$} & 114 & $354.2 \pm 8.2$ & $32.8 \pm 3.9$ & $47.6 \pm 4.2$ & $13.8 \pm 3.7$ & $0.0 \pm 2.8$ & $0.0 \pm 2.3$ & $2.3 \pm 2.0$ & $3.4 \pm 2.7$ & 677 & 2.2 & \\
\hline & 200 & $0.420 \pm 0.230$ & $8.4 \pm 10.0$ & $91.6 \pm 22.4$ & $0.0 \pm 14.1$ & $0.0 \pm 12.5$ & $0.0 \pm 6.9$ & $0.0 \pm 5.1$ & $0.0 \pm 7.6$ & 4.64 & $\ldots$ & \\
\hline \multirow{2}{*}{$\begin{array}{l}\text { CW Tau } \\
\text { "'” }\end{array}$} & 151 & $142.2 \pm 3.9$ & $0.0 \pm 26.8$ & $24.8 \pm 17.8$ & $0.0 \pm 21.1$ & $0.0 \pm 14.0$ & $15.5 \pm 12.7$ & $29.8 \pm 15.7$ & $29.9 \pm 17.3$ & 59.1 & 5.3 & \\
\hline & 659 & $1.52 \pm 0.03$ & $0.0 \pm 7.5$ & $51.6 \pm 11.7$ & $42.1 \pm 10.8$ & $0.0 \pm 12.4$ & $2.9 \pm 5.9$ & $0.0 \pm 5.2$ & $3.4 \pm 4.2$ & 0.643 & $\ldots$ & 1 \\
\hline \multirow{2}{*}{$\begin{array}{l}\text { CX Tau } \\
\text { "” }\end{array}$} & 151 & $58.3 \pm 1.4$ & $27.2 \pm 15.1$ & $62.7 \pm 19.8$ & $0.0 \pm 16.0$ & $0.0 \pm 11.5$ & $0.0 \pm 8.8$ & $3.4 \pm 7.5$ & $6.8 \pm 9.4$ & 27.0 & 2.6 & ป \\
\hline & 602 & $0.400 \pm 0.013$ & $7.3 \pm 6.8$ & $15.2 \pm 7.4$ & $24.4 \pm 9.9$ & $38.9 \pm 9.9$ & $7.6 \pm 5.4$ & $3.2 \pm 5.1$ & $3.4 \pm 4.2$ & 0.268 & $\ldots$ & \\
\hline \multirow{2}{*}{$\begin{array}{l}\text { CY Tau } \\
\text { “"” }\end{array}$} & 127 & $22.4 \pm 1.0$ & $22.6 \pm 37.7$ & $0.0 \pm 30.1$ & $0.0 \pm 34.0$ & $0.0 \pm 21.9$ & $0.0 \pm 20.2$ & $48.2 \pm 38.1$ & $29.2 \pm 28.6$ & 9.50 & 4.0 & \\
\hline & 602 & $0.319 \pm 0.006$ & $0.0 \pm 22.1$ & $69.9 \pm 39.9$ & $0.0 \pm 33.1$ & $0.0 \pm 30.1$ & $12.8 \pm 17.0$ & $0.0 \pm 13.839$ & $17.3 \pm 15.1$ & 0.0460 & $\ldots$ & \\
\hline \multirow{2}{*}{$\begin{array}{l}\text { DD Tau } \\
\text { "'" }\end{array}$} & 127 & $220.2 \pm 8.4$ & $80.5 \pm 18.1$ & $0.0 \pm 10.7$ & $0.0 \pm 11.8$ & $0.0 \pm 7.9$ & $10.1 \pm 6.8$ & $9.3 \pm 6.4$ & $0.0 \pm 9.0$ & 233 & 3.5 & \\
\hline & 488 & $4.72 \pm 0.07$ & $26.3 \pm 9.1$ & $0.0 \pm 9.7$ & $7.9 \pm 13.0$ & $46.2 \pm 12.3$ & $13.7 \pm 6.8$ & $5.8 \pm 6.1$ & $0.0 \pm 4.8$ & 1.42 & $\cdots$ & \\
\hline \multirow{2}{*}{$\begin{array}{l}\text { DE Tau } \\
\text { "'” }\end{array}$} & 115 & $226.5 \pm 7.7$ & $84.7 \pm 9.9$ & $5.2 \pm 5.6$ & $0.0 \pm 6.2$ & $0.0 \pm 4.0$ & $0.0 \pm 3.5$ & $10.1 \pm 3.4$ & $0.0 \pm 4.6$ & 390 & 4.7 & \\
\hline & 545 & $1.17 \pm 0.02$ & $0.0 \pm 6.1$ & $59.2 \pm 10.4$ & $0.0 \pm 10.1$ & $28.0 \pm 8.2$ & $5.0 \pm 4.8$ & $7.8 \pm 4.3$ & $0.0 \pm 3.4$ & 0.600 & $\ldots$ & \\
\hline \multirow{2}{*}{$\begin{array}{l}\text { DF Tau } \\
\text { ""” }\end{array}$} & 163 & $46.6 \pm 2.2$ & $0.0 \pm 14.1$ & $0.0 \pm 10.2$ & $0.0 \pm 12.3$ & $86.4 \pm 21.3$ & $0.0 \pm 6.8$ & $4.6 \pm 5.8$ & $9.0 \pm 8.0$ & 56.2 & 5.3 & \\
\hline & 716 & $1.34 \pm 0.02$ & $63.6 \pm 27.0$ & $0.0 \pm 17.4$ & $0.0 \pm 23.8$ & $0.0 \pm 23.4$ & $18.5 \pm 12.5$ & $17.9 \pm 11.6$ & $0.0 \pm 7.6$ & 0.250 & $\ldots$ & \\
\hline \multirow{2}{*}{$\begin{array}{l}\text { DG Tau } \\
\text { "'" }\end{array}$} & 127 & $2810.9 \pm 56.2$ & $86.3 \pm 18.4$ & $0.0 \pm 11.0$ & $0.0 \pm 11.3$ & $0.0 \pm 7.3$ & $0.0 \pm 5.8$ & $11.7 \pm 6.4$ & $1.9 \pm 8.2$ & 1350 & 6.4 & \\
\hline & 431 & $23.1 \pm 0.4$ & $0.0 \pm 335.1$ & $0.0 \pm 531.6$ & $0.0 \pm 745.9$ & $0.0 \pm 855.8$ & $0.0 \pm 326.0$ & $100.0 \pm 1347.6$ & $0.0 \pm 162.1$ & 0.113 & $\ldots$ & \\
\hline \multirow{2}{*}{$\begin{array}{l}\text { DH Tau } \\
\text { "'” }\end{array}$} & 127 & $89.4 \pm 2.8$ & $6.7 \pm 14.5$ & $48.9 \pm 13.4$ & $0.0 \pm 12.4$ & $0.0 \pm 8.5$ & $17.9 \pm 7.7$ & $26.6 \pm 8.9$ & $0.0 \pm 7.0$ & 68.3 & 8.4 & \\
\hline & 944 & $0.0953 \pm 0.0032$ & $0.0 \pm 4.1$ & $7.5 \pm 4.9$ & $0.0 \pm 7.4$ & $47.3 \pm 6.7$ & $26.0 \pm 4.5$ & $14.8 \pm 3.8$ & $4.3 \pm 2.1$ & 0.115 & $\ldots$ & \\
\hline \multirow{2}{*}{$\begin{array}{l}\text { DK Tau } \\
\text { "'” }\end{array}$} & 175 & $74.0 \pm 2.1$ & $0.0 \pm 14.0$ & $9.1 \pm 9.4$ & $19.0 \pm 11.3$ & $15.9 \pm 8.5$ & $17.1 \pm 7.4$ & $15.4 \pm 6.8$ & $23.4 \pm 9.1$ & 54.3 & 5.7 & \\
\hline & 830 & $0.631 \pm 0.020$ & $0.0 \pm 2.2$ & $1.5 \pm 2.6$ & $30.3 \pm 3.1$ & $54.2 \pm 3.6$ & $7.1 \pm 1.9$ & $4.8 \pm 1.8$ & $2.0 \pm 1.1$ & 1.58 & $\ldots$ & \\
\hline \multirow{2}{*}{$\begin{array}{l}\text { DL Tau } \\
\text { "'” }\end{array}$} & 139 & $147.2 \pm 5.2$ & $0.0 \pm 25.1$ & $0.0 \pm 17.3$ & $36.0 \pm 20.3$ & $41.0 \pm 20.0$ & $10.0 \pm 11.8$ & $6.8 \pm 10.6$ & $6.2 \pm 14.3$ & 75.1 & 3.8 & \\
\hline & 488 & $3.63 \pm 0.06$ & $16.2 \pm 22.6$ & $0.0 \pm 25.3$ & $0.0 \pm 38.2$ & $0.0 \pm 34.9$ & $34.4 \pm 25.8$ & $26.8 \pm 21.7$ & $22.6 \pm 18.7$ & 0.385 & $\cdots$ & \\
\hline \multirow{2}{*}{$\begin{array}{l}\text { DM Tau } \\
\text { (")" }\end{array}$} & 121 & $119.1 \pm 2.7$ & $0.0 \pm 15.5$ & $70.6 \pm 18.5$ & $0.0 \pm 13.6$ & $0.0 \pm 9.1$ & $0.0 \pm 6.1$ & $12.6 \pm 7.2$ & $16.9 \pm 8.3$ & 62.2 & 4.5 & \\
\hline & 260 & $0.295 \pm 0.040$ & $0.0 \pm 2.4$ & $25.3 \pm 2.7$ & $34.0 \pm 3.2$ & $40.4 \pm 3.3$ & $0.0 \pm 1.7$ & $0.2 \pm 1.7$ & $0.0 \pm 1.2$ & 2.90 & $\cdots$ & \\
\hline
\end{tabular}


Table 6-Continued

\begin{tabular}{|c|c|c|c|c|c|c|c|c|c|c|c|c|}
\hline $\begin{array}{l}\text { Object } \\
\text { (1) }\end{array}$ & $\begin{array}{c}\text { Temp } \\
(\mathrm{K}) \\
(2)\end{array}$ & $\begin{array}{c}\Omega_{B B} \\
(3)\end{array}$ & $\begin{array}{c}\text { small } \\
\text { Amorphous } \\
\text { Pyroxene } \\
\text { (4) }\end{array}$ & $\begin{array}{c}\text { small } \\
\text { Amorph. } \\
\text { Olivine }^{\mathrm{b}} \\
(5)\end{array}$ & $\begin{array}{c}\text { large } \\
\text { Amorph. } \\
\text { Pyroxene } \\
\quad(6)\end{array}$ & $\begin{array}{c}\text { large } \\
\text { Amorph. } \\
\text { Olivine }^{\mathrm{d}} \\
(7)\end{array}$ & $\begin{array}{c}\text { Crystalline } \\
\text { Enstatite }^{\mathrm{e}} \\
(8)\end{array}$ & $\begin{array}{c}\text { Crystalline } \\
\text { Forsterite }^{f} \\
(9)\end{array}$ & $\begin{array}{c}\text { Crystalline } \\
\text { Silica }^{\mathrm{g}} \\
(10)\end{array}$ & $\begin{array}{c}\text { Total } \\
\text { Dust } \\
\text { Mass } \\
(11)\end{array}$ & $\frac{\chi^{2}}{\text { d.o.f. }}$ & \\
\hline \multirow{2}{*}{$\begin{array}{l}\text { DN Tau } \\
\text { “"” }\end{array}$} & 127 & $100.1 \pm 3.6$ & $77.1 \pm 23.7$ & $16.4 \pm 13.5$ & $0.0 \pm 16.2$ & $0.0 \pm 10.9$ & $0.0 \pm 9.1$ & $5.9 \pm 8.1$ & $0.6 \pm 12.5$ & 68.4 & 2.9 & \\
\hline & 431 & $1.99 \pm 0.03$ & $0.0 \pm 20.5$ & $49.7 \pm 28.9$ & $0.0 \pm 31.9$ & $0.0 \pm 29.2$ & $20.6 \pm 17.0$ & $7.7 \pm 13.3$ & $22.0 \pm 15.8$ & 0.283 & $\ldots$ & \\
\hline \multirow{2}{*}{$\begin{array}{l}\text { DO Tau } \\
\text { "'” }\end{array}$} & 139 & $565.9 \pm 11.4$ & $79.4 \pm 35.5$ & $0.0 \pm 20.1$ & $0.0 \pm 25.1$ & $0.0 \pm 16.9$ & $0.0 \pm 13.4$ & $12.7 \pm 12.8$ & $7.9 \pm 15.9$ & 136 & 2.3 & \\
\hline & 545 & $3.59 \pm 0.07$ & $0.0 \pm 5.3$ & $1.3 \pm 6.3$ & $12.1 \pm 8.2$ & $76.8 \pm 11.0$ & $5.6 \pm 4.3$ & $4.2 \pm 4.0$ & $0.0 \pm 2.9$ & 2.03 & $\ldots$ & \\
\hline \multirow{2}{*}{$\begin{array}{l}\text { DP Tau } \\
\text { "'" }\end{array}$} & 139 & $145.5 \pm 4.8$ & $34.2 \pm 6.7$ & $50.1 \pm 7.3$ & $0.0 \pm 6.6$ & $0.0 \pm 4.4$ & $4.0 \pm 3.6$ & $4.7 \pm 3.2$ & $6.9 \pm 4.9$ & 236 & 2.9 & \\
\hline & 488 & $2.40 \pm 0.05$ & $0.0 \pm 6.0$ & $64.7 \pm 11.8$ & $0.0 \pm 10.5$ & $29.9 \pm 8.7$ & $0.0 \pm 5.1$ & $5.4 \pm 4.8$ & $0.0 \pm 2.9$ & 1.16 & $\cdots$ & \\
\hline \multirow{2}{*}{$\begin{array}{l}\text { DQ Tau } \\
\text { ""” }\end{array}$} & 127 & $236.4 \pm 7.1$ & $93.1 \pm 30.6$ & $0.0 \pm 15.0$ & $0.0 \pm 18.0$ & $0.0 \pm 11.9$ & $0.0 \pm 10.6$ & $6.9 \pm 9.9$ & $0.0 \pm 13.2$ & 122 & 3.1 & \\
\hline & 431 & $4.43 \pm 0.07$ & $8.1 \pm 16.9$ & $42.8 \pm 22.8$ & $0.0 \pm 27.6$ & $0.0 \pm 25.4$ & $41.1 \pm 21.6$ & $7.9 \pm 12.1$ & $0.0 \pm 9.3$ & 0.642 & $\ldots$ & \\
\hline \multirow{2}{*}{$\begin{array}{l}\text { DR Tau } \\
\text { "'” }\end{array}$} & 127 & $683.4 \pm 17.9$ & $47.6 \pm 10.8$ & $41.4 \pm 9.8$ & $0.0 \pm 10.0$ & $0.0 \pm 6.7$ & $0.0 \pm 5.5$ & $3.9 \pm 4.8$ & $7.0 \pm 6.8$ & 594 & 2.1 & \\
\hline & 545 & $6.24 \pm 0.10$ & $0.0 \pm 7.4$ & $59.0 \pm 12.5$ & $5.5 \pm 11.6$ & $34.5 \pm 9.9$ & $0.5 \pm 6.0$ & $0.5 \pm 5.4$ & $0.0 \pm 4.0$ & 2.32 & $\ldots$ & \\
\hline \multirow{2}{*}{$\begin{array}{l}\text { DS Tau } \\
\text { "" }\end{array}$} & 139 & $31.1 \pm 1.2$ & $0.0 \pm 25.1$ & $21.9 \pm 16.6$ & $40.8 \pm 20.2$ & $0.0 \pm 13.3$ & $21.1 \pm 13.0$ & $10.9 \pm 10.6$ & $5.3 \pm 13.5$ & 19.1 & 5.1 & \\
\hline & 545 & $0.674 \pm 0.014$ & $0.0 \pm 3.7$ & $45.3 \pm 5.0$ & $44.7 \pm 5.1$ & $0.0 \pm 5.2$ & $4.1 \pm 2.9$ & $3.8 \pm 2.7$ & $2.1 \pm 1.9$ & 0.682 & $\cdots$ & | \\
\hline \multirow{2}{*}{$\begin{array}{l}\text { F04147+2822 } \\
\text { "'" }\end{array}$} & 163 & $9.70 \pm 0.54$ & $0.0 \pm 8.1$ & $18.9 \pm 5.4$ & $61.1 \pm 8.5$ & $0.0 \pm 4.9$ & $4.2 \pm 3.8$ & $15.8 \pm 3.8$ & $0.0 \pm 4.7$ & 24.1 & 2.2 & $\infty$ \\
\hline & 602 & $0.361 \pm 0.009$ & $3.2 \pm 3.5$ & $36.2 \pm 4.1$ & $60.6 \pm 5.4$ & $0.0 \pm 4.9$ & $0.0 \pm 2.6$ & $0.0 \pm 2.4$ & $0.0 \pm 1.9$ & 0.485 & $\ldots$ & \\
\hline \multirow{2}{*}{$\begin{array}{l}\text { FM Tau } \\
\text { "'” }\end{array}$} & 127 & $93.4 \pm 3.0$ & $60.2 \pm 11.1$ & $27.1 \pm 7.9$ & $0.0 \pm 9.2$ & $0.0 \pm 6.2$ & $5.6 \pm 5.2$ & $3.5 \pm 4.6$ & $3.6 \pm 6.2$ & 107 & 2.4 & \\
\hline & 431 & $1.32 \pm 0.03$ & $7.6 \pm 4.4$ & $78.3 \pm 9.3$ & $10.5 \pm 6.4$ & $0.0 \pm 6.3$ & $1.6 \pm 3.4$ & $2.0 \pm 3.1$ & $0.0 \pm 2.4$ & 1.19 & $\ldots$ & \\
\hline \multirow{2}{*}{$\begin{array}{l}\text { FN Tau } \\
\text { “"” }\end{array}$} & 139 & $254.4 \pm 5.3$ & $74.6 \pm 19.7$ & $0.0 \pm 12.9$ & $0.0 \pm 14.3$ & $0.0 \pm 10.2$ & $0.1 \pm 8.6$ & $25.3 \pm 9.6$ & $0.0 \pm 7.0$ & 105 & 5.1 & \\
\hline & 488 & $1.80 \pm 0.04$ & $0.0 \pm 5.8$ & $38.2 \pm 7.8$ & $0.0 \pm 10.0$ & $30.6 \pm 8.3$ & $28.8 \pm 6.2$ & $2.4 \pm 4.6$ & $0.0 \pm 3.2$ & 1.11 & $\ldots$ & \\
\hline \multirow{2}{*}{$\begin{array}{l}\text { FO Tau } \\
\text { "'" }\end{array}$} & 115 & $133.8 \pm 6.0$ & $43.5 \pm 6.6$ & $23.8 \pm 5.2$ & $19.2 \pm 5.5$ & $0.0 \pm 4.2$ & $8.9 \pm 3.5$ & $4.6 \pm 3.2$ & $0.0 \pm 4.5$ & 332 & 2.7 & \\
\hline & 488 & $1.57 \pm 0.03$ & $42.6 \pm 11.8$ & $0.0 \pm 9.6$ & $40.2 \pm 12.5$ & $0.0 \pm 12.9$ & $8.1 \pm 7.0$ & $2.6 \pm 6.7$ & $6.5 \pm 5.5$ & 0.537 & $\ldots$ & \\
\hline \multirow{2}{*}{$\begin{array}{l}\text { FP Tau } \\
\text { "'" }\end{array}$} & 115 & $39.4 \pm 2.0$ & $13.2 \pm 6.4$ & $0.0 \pm 5.0$ & $82.2 \pm 9.4$ & $0.0 \pm 3.9$ & $1.4 \pm 3.1$ & $3.2 \pm 2.7$ & $0.0 \pm 4.3$ & 110 & 5.3 & \\
\hline & 488 & $0.400 \pm 0.008$ & $90.2 \pm 41.6$ & $0.0 \pm 18.4$ & $0.0 \pm 27.8$ & $0.0 \pm 24.7$ & $0.9 \pm 13.1$ & $8.9 \pm 11.7$ & $0.0 \pm 9.2$ & 0.0743 & $\ldots$ & \\
\hline \multirow{2}{*}{$\begin{array}{l}\text { FQ Tau } \\
\text { "'” }\end{array}$} & 151 & $12.7 \pm 0.4$ & $47.2 \pm 119.2$ & $0.0 \pm 95.8$ & $0.0 \pm 116.3$ & $0.0 \pm 80.9$ & $0.0 \pm 64.0$ & $51.7 \pm 125.2$ & $1.1 \pm 60.0$ & 1.02 & 3.9 & \\
\hline & 830 & $0.0635 \pm 0.0016$ & $10.0 \pm 6.0$ & $0.0 \pm 7.3$ & $4.6 \pm 10.5$ & $71.4 \pm 12.3$ & $11.9 \pm 5.2$ & $1.5 \pm 4.7$ & $0.6 \pm 3.7$ & 0.0345 & $\ldots$ & \\
\hline \multirow{2}{*}{$\begin{array}{l}\text { FS Tau } \\
\text { “"” }\end{array}$} & 127 & $445.3 \pm 12.2$ & $27.2 \pm 9.6$ & $18.9 \pm 7.5$ & $45.4 \pm 9.8$ & $0.0 \pm 6.5$ & $0.0 \pm 5.2$ & $3.6 \pm 4.5$ & $4.9 \pm 6.8$ & 415 & 2.8 & \\
\hline & 488 & $3.05 \pm 0.06$ & $0.0 \pm 6.1$ & $44.8 \pm 9.0$ & $8.2 \pm 9.9$ & $40.1 \pm 9.1$ & $2.7 \pm 5.1$ & $4.3 \pm 4.6$ & $0.0 \pm 3.2$ & 1.45 & $\ldots$ & \\
\hline \multirow{2}{*}{$\begin{array}{l}\text { FT Tau } \\
\text { (") }\end{array}$} & 115 & $101.0 \pm 4.2$ & $74.8 \pm 18.6$ & $10.5 \pm 11.1$ & $0.0 \pm 12.6$ & $0.0 \pm 8.2$ & $0.0 \pm 7.2$ & $13.3 \pm 7.0$ & $1.4 \pm 10.5$ & 108 & 5.9 & \\
\hline & 431 & $1.29 \pm 0.03$ & $7.5 \pm 6.5$ & $60.6 \pm 11.3$ & $15.4 \pm 9.4$ & $0.0 \pm 9.7$ & $7.4 \pm 5.2$ & $4.6 \pm 4.7$ & $4.4 \pm 3.8$ & 0.675 & $\ldots$ & \\
\hline \multirow{2}{*}{$\begin{array}{l}\text { FV Tau } \\
\text { (") }\end{array}$} & 127 & $359.6 \pm 13.4$ & $35.7 \pm 7.2$ & $58.7 \pm 8.6$ & $0.0 \pm 6.8$ & $0.0 \pm 4.5$ & $1.8 \pm 3.9$ & $1.1 \pm 3.5$ & $2.7 \pm 4.8$ & 652 & 3.6 & \\
\hline & 545 & $5.24 \pm 0.09$ & $0.0 \pm 3.5$ & $20.0 \pm 5.0$ & $0.0 \pm 6.8$ & $72.6 \pm 8.1$ & $0.0 \pm 3.6$ & $7.5 \pm 3.4$ & $0.0 \pm 1.5$ & 3.30 & $\ldots$ & \\
\hline \multirow{2}{*}{$\begin{array}{l}\text { FX Tau } \\
\text { "'” }\end{array}$} & 115 & $58.3 \pm 5.4$ & $0.0 \pm 6.5$ & $55.3 \pm 6.3$ & $0.0 \pm 5.2$ & $29.8 \pm 4.1$ & $1.3 \pm 3.0$ & $6.8 \pm 2.7$ & $6.7 \pm 3.8$ & 358 & 5.2 & \\
\hline & 545 & $0.788 \pm 0.019$ & $6.4 \pm 3.2$ & $48.1 \pm 4.6$ & $30.3 \pm 4.3$ & $0.7 \pm 4.7$ & $8.9 \pm 2.5$ & $5.5 \pm 2.4$ & $0.0 \pm 1.6$ & 1.10 & $\ldots$ & \\
\hline
\end{tabular}


Table 6-Continued

\begin{tabular}{|c|c|c|c|c|c|c|c|c|c|c|c|c|}
\hline $\begin{array}{l}\text { Object } \\
(1)\end{array}$ & $\begin{array}{c}\text { Temp } \\
(\mathrm{K}) \\
(2)\end{array}$ & $\begin{array}{c}\Omega_{B B} \\
(3)\end{array}$ & $\begin{array}{c}\text { small } \\
\text { Amorphous } \\
\text { Pyroxene } \\
\text { (4) }\end{array}$ & $\begin{array}{c}\text { small } \\
\text { Amorph. } \\
\text { Olivine }^{\mathrm{b}} \\
(5)\end{array}$ & $\begin{array}{c}\text { large } \\
\text { Amorph. } \\
\text { Pyroxene } \\
\quad(6)\end{array}$ & $\begin{array}{c}\text { large } \\
\text { Amorph. } \\
\text { Olivine }^{\mathrm{d}} \\
(7)\end{array}$ & $\begin{array}{c}\text { Crystalline } \\
\text { Enstatite }^{\mathrm{e}} \\
(8)\end{array}$ & $\begin{array}{c}\text { Crystalline } \\
\text { Forsterite }^{\mathrm{f}} \\
\quad(9)\end{array}$ & $\begin{array}{c}\text { Crystalline } \\
\text { Silica }^{\mathrm{g}} \\
(10)\end{array}$ & $\begin{array}{c}\text { Total } \\
\text { Dust } \\
\text { Mass } \\
(11)\end{array}$ & $\frac{\chi^{2}}{\text { d.o.f. }}$ & \\
\hline \multirow{2}{*}{$\begin{array}{l}\text { FZ Tau } \\
\text { "'” }\end{array}$} & 139 & $135.1 \pm 4.5$ & $0.0 \pm 32.6$ & $50.9 \pm 29.3$ & $0.0 \pm 26.4$ & $0.0 \pm 18.0$ & $21.6 \pm 16.8$ & $14.7 \pm 13.6$ & $12.8 \pm 17.5$ & 55.7 & 7.5 & \\
\hline & 545 & $2.68 \pm 0.05$ & $0.0 \pm 6.0$ & $0.0 \pm 6.5$ & $51.2 \pm 9.0$ & $27.2 \pm 7.9$ & $6.7 \pm 4.7$ & $4.9 \pm 4.3$ & $10.0 \pm 3.9$ & 1.29 & $\ldots$ & \\
\hline \multirow{2}{*}{$\begin{array}{l}\text { GG Tau A } \\
\text { "'" }\end{array}$} & 139 & $192.2 \pm 5.0$ & $73.2 \pm 16.0$ & $8.6 \pm 9.7$ & $0.0 \pm 11.9$ & $0.0 \pm 8.1$ & $0.0 \pm 6.5$ & $12.8 \pm 6.6$ & $5.4 \pm 6.9$ & 130 & 2.4 & \\
\hline & 545 & $1.65 \pm 0.04$ & $0.0 \pm 2.9$ & $35.7 \pm 3.5$ & $49.3 \pm 4.2$ & $12.5 \pm 3.9$ & $0.3 \pm 2.3$ & $0.9 \pm 2.2$ & $1.3 \pm 1.5$ & 2.44 & $\cdots$ & \\
\hline \multirow{2}{*}{$\begin{array}{l}\text { GG Tau B } \\
\text { ""” }\end{array}$} & 139 & $46.1 \pm 1.0$ & $51.6 \pm 8.7$ & $37.1 \pm 7.2$ & $0.0 \pm 8.0$ & $0.0 \pm 5.5$ & $0.0 \pm 4.3$ & $8.7 \pm 4.2$ & $2.7 \pm 4.8$ & 38.2 & 7.5 & \\
\hline & 1172 & $0.0224 \pm 0.0009$ & $0.0 \pm 3.4$ & $12.6 \pm 4.2$ & $25.5 \pm 5.3$ & $43.1 \pm 5.6$ & $12.7 \pm 3.1$ & $6.2 \pm 2.9$ & $0.0 \pm 1.5$ & 0.0438 & $\ldots$ & \\
\hline \multirow{2}{*}{$\begin{array}{l}\text { GH Tau } \\
\text { "'" }\end{array}$} & 139 & $65.8 \pm 2.0$ & $80.8 \pm 15.9$ & $0.0 \pm 9.1$ & $0.0 \pm 10.7$ & $0.0 \pm 7.2$ & $0.0 \pm 5.9$ & $14.0 \pm 5.8$ & $5.2 \pm 7.5$ & 56.5 & 4.3 & \\
\hline & 716 & $0.349 \pm 0.007$ & $0.0 \pm 6.3$ & $1.3 \pm 7.3$ & $10.5 \pm 9.6$ & $76.8 \pm 12.8$ & $7.7 \pm 5.0$ & $0.6 \pm 4.6$ & $3.2 \pm 3.5$ & 0.182 & $\ldots$ & \\
\hline \multirow{2}{*}{$\begin{array}{l}\text { GI Tau } \\
\text { ""” }\end{array}$} & 115 & $171.6 \pm 10.1$ & $0.0 \pm 7.6$ & $0.0 \pm 5.7$ & $87.1 \pm 11.0$ & $0.0 \pm 4.0$ & $9.2 \pm 3.5$ & $3.3 \pm 3.2$ & $0.4 \pm 5.4$ & 610 & 3.9 & \\
\hline & 488 & $2.99 \pm 0.06$ & $19.9 \pm 3.5$ & $38.5 \pm 4.3$ & $9.2 \pm 5.1$ & $29.3 \pm 4.3$ & $1.5 \pm 2.7$ & $1.6 \pm 2.4$ & $0.0 \pm 1.8$ & 3.22 & $\ldots$ & \\
\hline \multirow{2}{*}{$\begin{array}{l}\text { GK Tau } \\
\text { "'” }\end{array}$} & 175 & $115.6 \pm 2.6$ & $0.0 \pm 10.0$ & $0.0 \pm 7.2$ & $38.7 \pm 8.6$ & $35.1 \pm 7.6$ & $10.5 \pm 4.9$ & $9.8 \pm 4.5$ & $6.0 \pm 5.8$ & 90.4 & 4.2 & \\
\hline & 1001 & $0.283 \pm 0.013$ & $0.0 \pm 2.3$ & $40.3 \pm 3.3$ & $45.6 \pm 3.6$ & $6.6 \pm 3.9$ & $3.8 \pm 2.0$ & $3.4 \pm 2.0$ & $0.3 \pm 1.0$ & 0.992 & $\ldots$ & 1 \\
\hline \multirow{2}{*}{$\begin{array}{l}\text { GM Aur } \\
\text { "'" }\end{array}$} & 91 & $810.0 \pm 32.8$ & $58.4 \pm 3.8$ & $37.7 \pm 3.0$ & $0.0 \pm 3.1$ & $0.0 \pm 2.0$ & $0.0 \pm 1.7$ & $2.1 \pm 1.7$ & $1.9 \pm 2.5$ & 3610 & 4.9 & $\infty$ \\
\hline & 488 & $0.326 \pm 0.014$ & $1.0 \pm 3.5$ & $98.1 \pm 8.8$ & $0.0 \pm 5.3$ & $0.0 \pm 4.9$ & $0.0 \pm 2.5$ & $0.9 \pm 2.6$ & $0.0 \pm 1.6$ & 0.714 & $\ldots$ & $\longmapsto$ \\
\hline \multirow{2}{*}{$\begin{array}{l}\text { GN Tau } \\
\text { "'” }\end{array}$} & 139 & $48.9 \pm 2.2$ & $0.0 \pm 18.2$ & $36.4 \pm 14.0$ & $0.0 \pm 14.3$ & $0.0 \pm 9.8$ & $33.6 \pm 12.3$ & $19.9 \pm 9.3$ & $10.1 \pm 11.4$ & 50.7 & 5.6 & 1 \\
\hline & 545 & $1.06 \pm 0.02$ & $0.0 \pm 3.1$ & $12.2 \pm 3.1$ & $53.7 \pm 4.5$ & $22.7 \pm 3.8$ & $1.1 \pm 2.4$ & $2.8 \pm 2.1$ & $7.6 \pm 1.8$ & 1.42 & $\cdots$ & \\
\hline \multirow{2}{*}{$\begin{array}{l}\text { GO Tau } \\
\text { “"” }\end{array}$} & 115 & $59.1 \pm 1.9$ & $86.5 \pm 17.5$ & $0.0 \pm 9.9$ & $0.0 \pm 10.9$ & $0.0 \pm 7.0$ & $0.0 \pm 6.1$ & $11.2 \pm 5.8$ & $2.3 \pm 8.1$ & 53.2 & 5.3 & \\
\hline & 545 & $0.213 \pm 0.006$ & $0.0 \pm 5.1$ & $34.2 \pm 6.0$ & $42.1 \pm 7.1$ & $14.3 \pm 7.1$ & $4.3 \pm 3.9$ & $3.1 \pm 3.3$ & $2.0 \pm 2.8$ & 0.184 & $\ldots$ & \\
\hline \multirow{2}{*}{$\begin{array}{l}\text { Haro 6-28 } \\
\text { “"” }\end{array}$} & 163 & $14.9 \pm 0.6$ & $0.0 \pm 13.5$ & $0.0 \pm 9.6$ & $0.0 \pm 12.7$ & $82.4 \pm 19.9$ & $0.0 \pm 6.7$ & $6.0 \pm 5.7$ & $11.6 \pm 7.4$ & 15.1 & 11.8 & \\
\hline & 659 & $0.197 \pm 0.006$ & $11.3 \pm 9.5$ & $0.0 \pm 11.1$ & $44.2 \pm 14.8$ & $0.0 \pm 16.4$ & $21.4 \pm 9.0$ & $14.5 \pm 7.8$ & $8.6 \pm 6.1$ & 0.0825 & $\ldots$ & \\
\hline \multirow{2}{*}{$\begin{array}{l}\text { Haro 6-37 } \\
\text { (") }\end{array}$} & 163 & $74.0 \pm 2.0$ & $61.0 \pm 26.4$ & $1.2 \pm 18.9$ & $0.0 \pm 22.5$ & $0.0 \pm 15.5$ & $23.3 \pm 14.4$ & $11.1 \pm 11.1$ & $3.4 \pm 13.5$ & 26.5 & 4.9 & \\
\hline & 716 & $0.939 \pm 0.022$ & $6.9 \pm 6.5$ & $28.3 \pm 8.0$ & $0.0 \pm 11.6$ & $36.9 \pm 9.8$ & $18.8 \pm 6.0$ & $3.9 \pm 4.9$ & $5.0 \pm 3.8$ & 0.464 & $\ldots$ & \\
\hline \multirow{2}{*}{$\begin{array}{l}\text { HK Tau } \\
\text { "'” }\end{array}$} & 115 & $360.7 \pm 9.4$ & $91.6 \pm 10.0$ & $0.0 \pm 5.1$ & $0.0 \pm 5.7$ & $0.0 \pm 3.7$ & $0.0 \pm 3.4$ & $8.3 \pm 3.1$ & $0.1 \pm 5.0$ & 506 & 6.2 & \\
\hline & 488 & $0.809 \pm 0.020$ & $0.0 \pm 6.2$ & $14.3 \pm 7.1$ & $13.1 \pm 9.9$ & $48.3 \pm 9.9$ & $20.8 \pm 5.9$ & $3.5 \pm 4.8$ & $0.0 \pm 3.5$ & 0.483 & $\ldots$ & \\
\hline \multirow{2}{*}{$\begin{array}{l}\text { HN Tau } \\
\text { "'” }\end{array}$} & 139 & $171.0 \pm 5.1$ & $66.6 \pm 12.6$ & $0.0 \pm 8.8$ & $0.0 \pm 9.7$ & $0.0 \pm 6.6$ & $0.0 \pm 5.5$ & $20.2 \pm 5.9$ & $13.2 \pm 6.6$ & 165 & 4.5 & \\
\hline & 545 & $2.01 \pm 0.04$ & $0.0 \pm 3.6$ & $44.6 \pm 5.0$ & $22.8 \pm 5.0$ & $28.1 \pm 4.8$ & $0.0 \pm 2.9$ & $3.0 \pm 2.7$ & $1.5 \pm 1.9$ & 2.07 & $\ldots$ & \\
\hline \multirow{2}{*}{$\begin{array}{l}\text { HO Tau } \\
\text { "'” }\end{array}$} & 139 & $11.7 \pm 0.6$ & $82.7 \pm 30.1$ & $0.0 \pm 15.9$ & $0.0 \pm 19.0$ & $0.0 \pm 12.1$ & $0.0 \pm 10.2$ & $16.9 \pm 11.3$ & $0.4 \pm 17.9$ & 9.81 & 5.6 & \\
\hline & 488 & $0.211 \pm 0.006$ & $0.1 \pm 5.8$ & $53.0 \pm 8.6$ & $32.9 \pm 7.6$ & $0.0 \pm 8.2$ & $8.3 \pm 4.4$ & $3.9 \pm 3.8$ & $1.8 \pm 3.1$ & 0.178 & $\ldots$ & \\
\hline \multirow{2}{*}{$\begin{array}{l}\text { HP Tau } \\
\text { "'” }\end{array}$} & 139 & $386.8 \pm 9.8$ & $88.5 \pm 31.8$ & $0.0 \pm 15.5$ & $0.0 \pm 18.5$ & $0.0 \pm 12.2$ & $0.0 \pm 10.5$ & $11.5 \pm 10.3$ & $0.0 \pm 18.4$ & 178 & 2.1 & \\
\hline & 488 & $2.98 \pm 0.07$ & $0.0 \pm 3.5$ & $52.8 \pm 5.6$ & $28.4 \pm 4.9$ & $14.6 \pm 5.0$ & $0.0 \pm 2.9$ & $1.8 \pm 2.6$ & $2.3 \pm 1.8$ & 3.55 & $\ldots$ & \\
\hline \multirow{2}{*}{$\begin{array}{l}\text { HQ Tau } \\
\text { “"” }\end{array}$} & 187 & $77.3 \pm 2.1$ & $23.2 \pm 5.9$ & $25.7 \pm 4.8$ & $46.4 \pm 6.2$ & $0.0 \pm 4.3$ & $0.0 \pm 3.4$ & $3.2 \pm 3.0$ & $1.5 \pm 4.1$ & 108 & 4.9 & \\
\hline & 1400 & $0.227 \pm 0.008$ & $0.0 \pm 2.9$ & $46.5 \pm 4.5$ & $24.5 \pm 4.3$ & $25.7 \pm 4.4$ & $1.8 \pm 2.5$ & $1.4 \pm 2.5$ & $0.0 \pm 1.4$ & 0.480 & $\cdots$ & \\
\hline
\end{tabular}


Table 6-Continued

\begin{tabular}{|c|c|c|c|c|c|c|c|c|c|c|c|c|}
\hline $\begin{array}{l}\text { Object } \\
(1)\end{array}$ & $\begin{array}{l}\text { Temp } \\
(\mathrm{K}) \\
(2)\end{array}$ & $\begin{array}{c}\Omega_{B B} \\
(3)\end{array}$ & $\begin{array}{c}\text { small } \\
\text { Amorphous } \\
\text { Pyroxene }^{\mathrm{a}} \\
(4)\end{array}$ & $\begin{array}{c}\text { small } \\
\text { Amorph. } \\
\text { Olivine }^{\mathrm{b}} \\
(5)\end{array}$ & $\begin{array}{c}\text { large } \\
\text { Amorph. } \\
\text { Pyroxene }^{\mathrm{c}} \\
(6)\end{array}$ & $\begin{array}{c}\text { large } \\
\text { Amorph. } \\
\text { Olivine }^{\mathrm{d}} \\
(7)\end{array}$ & $\begin{array}{c}\text { Crystalline } \\
\text { Enstatite }^{\mathrm{e}} \\
(8)\end{array}$ & $\begin{array}{l}\text { Crystalline } \\
\text { Forsterite }^{\mathrm{f}} \\
(9)\end{array}$ & $\begin{array}{l}\text { Crystalline } \\
\text { Silica }^{\mathrm{g}} \\
(10)\end{array}$ & $\begin{array}{l}\text { Total } \\
\text { Dust } \\
\text { Mass } \\
(11)\end{array}$ & $\frac{\chi^{2}}{\text { d.o.f. }}$ & \\
\hline \multirow{2}{*}{$\begin{array}{l}\text { IP Tau } \\
\text { "'” }\end{array}$} & 103 & $88.6 \pm 6.3$ & $0.0 \pm 5.4$ & $27.7 \pm 3.9$ & $64.4 \pm 6.0$ & $0.0 \pm 2.7$ & $2.6 \pm 2.5$ & $0.8 \pm 2.1$ & $4.4 \pm 4.1$ & 546 & 4.2 & \\
\hline & 488 & $0.648 \pm 0.016$ & $23.7 \pm 3.9$ & $64.9 \pm 6.8$ & $5.9 \pm 5.8$ & $0.0 \pm 5.5$ & $1.4 \pm 3.1$ & $4.2 \pm 2.7$ & $0.0 \pm 1.9$ & 0.785 & $\ldots$ & \\
\hline \multirow{2}{*}{$\begin{array}{l}\text { IQ Tau } \\
\text { "'” }\end{array}$} & 139 & $51.8 \pm 1.9$ & $92.6 \pm 54.1$ & $0.0 \pm 29.2$ & $0.0 \pm 33.0$ & $0.0 \pm 21.9$ & $0.0 \pm 17.1$ & $7.4 \pm 17.6$ & $0.0 \pm 18.9$ & 16.9 & 6.3 & \\
\hline & 602 & $0.782 \pm 0.016$ & $0.0 \pm 4.3$ & $37.0 \pm 5.4$ & $10.7 \pm 6.5$ & $43.2 \pm 6.1$ & $4.2 \pm 3.5$ & $4.8 \pm 3.1$ & $0.0 \pm 2.3$ & 0.588 & $\cdots$ & \\
\hline \multirow{2}{*}{$\begin{array}{l}\text { IS Tau } \\
\text { "'” }\end{array}$} & 175 & $13.7 \pm 0.5$ & $0.0 \pm 31.1$ & $0.0 \pm 22.0$ & $0.0 \pm 26.8$ & $0.0 \pm 19.0$ & $3.8 \pm 15.2$ & $64.3 \pm 36.2$ & $31.9 \pm 21.4$ & 5.39 & 3.3 & \\
\hline & 659 & $0.417 \pm 0.010$ & $0.0 \pm 3.5$ & $0.0 \pm 3.7$ & $41.8 \pm 4.8$ & $44.0 \pm 4.8$ & $7.1 \pm 2.7$ & $2.8 \pm 2.5$ & $4.2 \pm 2.0$ & 0.504 & $\cdots$ & \\
\hline \multirow{2}{*}{$\begin{array}{l}\text { IT Tau } \\
\text { "'” }\end{array}$} & 127 & $53.5 \pm 2.3$ & $91.6 \pm 17.3$ & $0.0 \pm 9.3$ & $0.0 \pm 10.3$ & $0.0 \pm 7.0$ & $3.3 \pm 5.7$ & $4.9 \pm 5.1$ & $0.2 \pm 7.3$ & 72.8 & 6.3 & \\
\hline & 659 & $0.649 \pm 0.011$ & $0.0 \pm 25.3$ & $37.1 \pm 30.8$ & $0.0 \pm 40.4$ & $0.0 \pm 38.7$ & $52.6 \pm 38.6$ & $10.4 \pm 17.6$ & $0.0 \pm 13.8$ & 0.0709 & $\cdots$ & \\
\hline \multirow{2}{*}{$\begin{array}{l}\mathrm{Lk} \text { Ca } 15 \\
\text { “"” }\end{array}$} & 103 & $267.6 \pm 8.7$ & $86.5 \pm 8.0$ & $0.0 \pm 4.5$ & $0.0 \pm 4.9$ & $0.0 \pm 2.9$ & $0.0 \pm 2.6$ & $6.2 \pm 2.8$ & $7.4 \pm 3.8$ & 562 & 7.5 & \\
\hline & 716 & $0.182 \pm 0.006$ & $0.0 \pm 2.5$ & $88.7 \pm 6.2$ & $9.7 \pm 3.8$ & $0.0 \pm 3.9$ & $0.0 \pm 2.0$ & $1.6 \pm 2.0$ & $0.0 \pm 1.1$ & 0.451 & $\ldots$ & \\
\hline \multirow{2}{*}{$\begin{array}{l}\text { RW Aur A } \\
\text { "'” }\end{array}$} & 151 & $230.2 \pm 5.5$ & $67.2 \pm 25.0$ & $0.0 \pm 16.0$ & $0.0 \pm 19.5$ & $0.0 \pm 13.0$ & $0.0 \pm 10.8$ & $18.5 \pm 11.1$ & $14.2 \pm 14.4$ & 88.3 & 3.9 & \\
\hline & 602 & $2.49 \pm 0.05$ & $4.1 \pm 6.2$ & $68.1 \pm 12.2$ & $10.9 \pm 9.4$ & $11.9 \pm 9.0$ & $3.8 \pm 5.0$ & $1.2 \pm 4.6$ & $0.0 \pm 3.5$ & 1.29 & $\cdots$ & 1 \\
\hline \multirow{2}{*}{$\begin{array}{l}\text { UY Aur } \\
\text { "'” }\end{array}$} & 151 & $912.9 \pm 16.9$ & $10.3 \pm 19.5$ & $0.0 \pm 15.2$ & $72.4 \pm 25.7$ & $0.0 \pm 13.1$ & $0.0 \pm 10.1$ & $4.6 \pm 8.9$ & $12.7 \pm 11.9$ & 284 & 1.4 & $\infty$ \\
\hline & 545 & $4.96 \pm 0.13$ & $0.0 \pm 3.6$ & $59.4 \pm 6.3$ & $25.9 \pm 5.1$ & $14.7 \pm 5.3$ & $0.0 \pm 2.9$ & $0.0 \pm 2.6$ & $0.0 \pm 1.7$ & 6.23 & $\ldots$ & N \\
\hline \multirow{2}{*}{$\begin{array}{l}\text { UZ Tau E } \\
\text { (") }\end{array}$} & 175 & $93.0 \pm 2.4$ & $47.8 \pm 10.4$ & $16.3 \pm 7.5$ & $30.6 \pm 9.0$ & $0.0 \pm 6.8$ & $1.0 \pm 5.3$ & $1.6 \pm 4.6$ & $2.8 \pm 6.8$ & 77.3 & 5.4 & | \\
\hline & 1400 & $0.282 \pm 0.009$ & $0.0 \pm 3.1$ & $7.0 \pm 3.7$ & $4.8 \pm 5.3$ & $85.9 \pm 7.3$ & $0.1 \pm 2.7$ & $2.3 \pm 2.6$ & $0.0 \pm 1.6$ & 0.401 & $\cdots$ & \\
\hline \multirow{2}{*}{$\begin{array}{l}\text { V410 Anon } 13 \\
\text { “"” }\end{array}$} & 103 & $7.28 \pm 0.65$ & $54.4 \pm 6.8$ & $6.8 \pm 5.0$ & $33.0 \pm 5.4$ & $0.0 \pm 3.6$ & $1.0 \pm 3.3$ & $4.3 \pm 3.0$ & $0.4 \pm 5.1$ & 42.5 & 5.6 & \\
\hline & 488 & $0.101 \pm 0.002$ & $46.3 \pm 13.6$ & $0.0 \pm 10.5$ & $10.4 \pm 14.4$ & $0.0 \pm 14.0$ & $24.0 \pm 8.9$ & $13.2 \pm 7.0$ & $6.1 \pm 6.4$ & 0.0318 & $\ldots$ & \\
\hline \multirow{2}{*}{$\begin{array}{l}\text { V710 Tau } \\
\text { "'” }\end{array}$} & 139 & $35.5 \pm 1.0$ & $62.9 \pm 23.1$ & $0.0 \pm 15.7$ & $0.0 \pm 18.1$ & $0.0 \pm 11.6$ & $0.0 \pm 10.2$ & $14.7 \pm 10.2$ & $22.4 \pm 14.8$ & 16.0 & 8.7 & \\
\hline & 602 & $0.427 \pm 0.008$ & $0.0 \pm 8.0$ & $63.4 \pm 13.8$ & $0.0 \pm 12.4$ & $14.1 \pm 10.3$ & $14.5 \pm 6.2$ & $2.4 \pm 5.5$ & $5.6 \pm 5.3$ & 0.176 & $\ldots$ & \\
\hline \multirow{2}{*}{$\begin{array}{l}\text { V807 Tau } \\
\text { “"” }\end{array}$} & 127 & $117.4 \pm 3.2$ & $54.9 \pm 7.9$ & $30.5 \pm 6.0$ & $0.0 \pm 7.0$ & $0.0 \pm 4.7$ & $0.0 \pm 3.7$ & $8.6 \pm 3.6$ & $6.0 \pm 4.5$ & 137 & 3.4 & \\
\hline & 773 & $0.428 \pm 0.008$ & $0.0 \pm 14.6$ & $8.9 \pm 16.2$ & $83.4 \pm 32.5$ & $0.0 \pm 25.1$ & $2.4 \pm 12.3$ & $5.2 \pm 10.6$ & $0.0 \pm 8.6$ & 0.0752 & $\cdots$ & \\
\hline \multirow{2}{*}{$\begin{array}{l}\text { V836 Tau } \\
\text { “"” }\end{array}$} & 127 & $37.1 \pm 1.4$ & $58.4 \pm 16.0$ & $0.0 \pm 12.1$ & $0.0 \pm 13.7$ & $0.0 \pm 9.2$ & $15.8 \pm 7.9$ & $16.5 \pm 7.6$ & $9.3 \pm 8.6$ & 32.6 & 4.5 & \\
\hline & 602 & $0.237 \pm 0.007$ & $0.0 \pm 3.3$ & $24.0 \pm 3.4$ & $55.8 \pm 4.8$ & $3.7 \pm 4.4$ & $7.8 \pm 2.4$ & $4.3 \pm 2.2$ & $4.5 \pm 1.8$ & 0.387 & $\ldots$ & \\
\hline \multirow{2}{*}{$\begin{array}{l}\text { V955 Tau } \\
\text { "'” }\end{array}$} & 115 & $81.8 \pm 5.9$ & $30.0 \pm 8.4$ & $49.4 \pm 9.0$ & $0.0 \pm 7.8$ & $0.0 \pm 5.2$ & $3.5 \pm 4.6$ & $15.4 \pm 4.7$ & $1.7 \pm 6.5$ & 257 & 2.5 & \\
\hline & 488 & $2.06 \pm 0.03$ & $12.3 \pm 6.1$ & $0.0 \pm 6.5$ & $63.0 \pm 10.0$ & $0.0 \pm 8.6$ & $11.5 \pm 4.8$ & $4.8 \pm 4.3$ & $8.4 \pm 3.9$ & 0.966 & $\ldots$ & \\
\hline \multirow{2}{*}{ VY Tau } & 115 & $47.7 \pm 1.7$ & $62.9 \pm 38.7$ & $0.0 \pm 30.0$ & $0.0 \pm 31.3$ & $0.0 \pm 22.0$ & $0.0 \pm 17.1$ & $37.1 \pm 27.4$ & $0.0 \pm 15.5$ & 15.3 & 7.2 & \\
\hline & 545 & $0.226 \pm 0.006$ & $22.0 \pm 6.2$ & $19.7 \pm 6.5$ & $43.8 \pm 8.7$ & $0.0 \pm 9.1$ & $5.0 \pm 4.8$ & $8.0 \pm 4.5$ & $1.5 \pm 3.6$ & 0.153 & $\cdots$ & \\
\hline \multirow{2}{*}{$\begin{array}{l}\text { XZ Tau } \\
\text { "'" }\end{array}$} & 151 & $787.4 \pm 18.1$ & $75.3 \pm 22.1$ & $20.5 \pm 12.5$ & $0.0 \pm 16.8$ & $0.0 \pm 12.2$ & $0.0 \pm 8.3$ & $4.2 \pm 7.8$ & $0.0 \pm 8.9$ & 317 & 2.0 & \\
\hline & 488 & $12.1 \pm 0.2$ & $0.0 \pm 9.6$ & $13.4 \pm 10.4$ & $68.3 \pm 17.7$ & $10.4 \pm 14.4$ & $4.8 \pm 7.7$ & $3.0 \pm 7.0$ & $0.0 \pm 5.5$ & 3.54 & $\ldots$ & \\
\hline ZZ Tau & 211 & $6.43 \pm 0.15$ & $0.0 \pm 33.3$ & $49.4 \pm 34.8$ & $0.0 \pm 35.2$ & $0.0 \pm 28.2$ & $0.4 \pm 18.9$ & $0.0 \pm 15.6$ & $50.1 \pm 35.1$ & 1.17 & 3.5 & \\
\hline “"' & 1400 & $0.0106 \pm 0.0007$ & $8.6 \pm 4.3$ & $0.0 \pm 5.4$ & $78.9 \pm 10.1$ & $0.0 \pm 8.5$ & $7.0 \pm 3.8$ & $1.2 \pm 3.7$ & $4.3 \pm 2.4$ & 0.0218 & $\cdots$ & \\
\hline
\end{tabular}


Table 6-Continued

\begin{tabular}{|c|c|c|c|c|c|c|c|c|c|c|c|}
\hline $\begin{array}{l}\text { Object } \\
(1)\end{array}$ & $\begin{array}{c}\text { Temp } \\
(\mathrm{K}) \\
(2)\end{array}$ & $\begin{array}{c}\Omega_{B B} \\
(3)\end{array}$ & $\begin{array}{c}\text { small } \\
\text { Amorphous } \\
\text { Pyroxene }^{\mathrm{a}} \\
(4)\end{array}$ & $\begin{array}{c}\text { small } \\
\text { Amorph. } \\
\text { Olivine }^{\mathrm{b}} \\
(5)\end{array}$ & $\begin{array}{c}\text { large } \\
\text { Amorph. } \\
\text { Pyroxene } \\
\quad(6)\end{array}$ & $\begin{array}{c}\text { large } \\
\text { Amorph. } \\
\text { Olivine }^{\mathrm{d}} \\
(7)\end{array}$ & $\begin{array}{c}\text { Crystalline } \\
\text { Enstatite }^{\mathrm{e}} \\
(8)\end{array}$ & $\begin{array}{c}\text { Crystalline } \\
\text { Forsterite }^{\text {f }} \\
(9)\end{array}$ & $\begin{array}{c}\text { Crystalline } \\
\text { Silicag }^{\mathrm{g}} \\
(10)\end{array}$ & $\begin{array}{l}\text { Total } \\
\text { Dust } \\
\text { Mass } \\
(11)\end{array}$ & 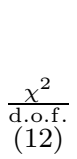 \\
\hline $\begin{array}{l}\text { ZZ Tau IRS } \\
\text { “"” }\end{array}$ & $\begin{array}{l}127 \\
545\end{array}$ & $\begin{array}{l}280.3 \pm 7.5 \\
1.28 \pm 0.03\end{array}$ & $\begin{aligned} 84.4 & \pm 11.1 \\
0.0 & \pm 3.1\end{aligned}$ & $\begin{array}{c}11.0 \pm 5.9 \\
0.0 \pm 4.1\end{array}$ & $\begin{array}{l}0.0 \pm 7.3 \\
0.0 \pm 6.2\end{array}$ & $\begin{array}{c}0.0 \pm 4.8 \\
96.3 \pm 8.8\end{array}$ & $\begin{array}{l}0.0 \pm 3.7 \\
0.0 \pm 2.9\end{array}$ & $\begin{array}{l}4.6 \pm 3.9 \\
3.7 \pm 2.8\end{array}$ & $\begin{array}{l}0.0 \pm 4.7 \\
0.0 \pm 1.5\end{array}$ & $\begin{array}{c}323 \\
1.18\end{array}$ & $\begin{array}{l}4.9 \\
\ldots\end{array}$ \\
\hline
\end{tabular}

Note. - Col. (1): Object name. Col. (2): One of two dust model temperatures (Kelvin). Col. (3): Solid angle, $\Omega_{B B}$, of blackbody of temperature specified in Col. (2) representing continuum emission, expressed in units of $10^{-16}$ steradians. Cols. (4)-(10): mass percentages and their propagated uncertainties of all dust masses at temperature specified in Col. (2). One dust model is completely specified by two adjacent rows - the row following the object's name and the row beneath that one. Propagated uncertainties are the square root of the sum of the squares of terms, such that each term is the product of the uncertainty in one of seven mass weights and the partial derivative of the mass fraction of the dust species in question with respect to the same mass weight. This way, even if a species has zero mass, its uncertainty still contributes to the propagated uncertainty in question. Col. (11): Total dust máss at one temperature in $10^{-4}$ lunar masses, computed assuming 140pc to Taurus-Auriga. Col. (12): $\chi^{2}$ per degree of freedom, determined over $7.7<\lambda<37 \mu \mathrm{m}$.

aOptical constants for amorphous pyroxene of cosmic composition from Jaeger et al. (1994), assuming CDE2 (Fabian et al. 2001)

${ }^{b}$ Optical constants for amorphous olivine $\mathrm{MgFeSiO}_{4}$ from Dorschner et al. (1995), assuming CDE2

${ }^{c}$ Optical constants for amorphous pyroxene of cosmic composition from Jaeger et al. (1994), using the Bruggeman EMT and Mie theory (Bohren \& Huffman 1983 ) with a volume fraction of vacuum of $f=0.6$ for porous spherical grains of radius $5 \mu \mathrm{m}$

${ }^{d}$ Optical constants for amorphous olivine $\mathrm{MgFeSiO}_{4}$ from Dorschner et al. (1995), using the Bruggeman EMT and Mie theory (Bohren \& Huffman 1983) with a volume fraction of vacuum of $f=0.6$ for porous spherical grains of radius $5 \mu \mathrm{m}$

e Opacities for clinoenstatite $\mathrm{Mg}_{0.9} \mathrm{Fe}_{0.1} \mathrm{SiO}_{3}$ from Chihara et al. (2002)

${ }^{\mathrm{f}}$ Optical constants for 3 crystallographic axes of forsterite, $\mathrm{Mg}_{2} \mathrm{SiO}_{4}$, from Sogawa et al. (2006), assuming tCDE (see discussion in Section 4.2.3).

gOpacity for annealed silica by Fabian et al. (2000). 
Table A1: Mispointing Corrections

\begin{tabular}{|c|c|c|c|c|c|c|c|c|c|}
\hline $\begin{array}{l}\text { Object } \\
(1)\end{array}$ & $\begin{array}{l}\text { modules } \\
(2)\end{array}$ & $\begin{array}{c}\text { SL2nod } 1 \\
\text { (3) }\end{array}$ & $\begin{array}{c}\text { SL2nod } 2 \\
\text { (4) }\end{array}$ & $\begin{array}{c}\text { SL1nod } 1 \\
(5)\end{array}$ & $\begin{array}{c}\text { SL1nod2 } \\
(6)\end{array}$ & $\begin{array}{l}\text { LL2nod1 or } \\
\text { SHnod } 1 \\
(7)\end{array}$ & $\begin{array}{c}\text { LL2nod2 or } \\
\text { SHnod2 } \\
(8)\end{array}$ & $\begin{array}{c}\text { LL1nod1 or } \\
\text { LHnod } 1 \\
(9)\end{array}$ & $\begin{array}{c}\text { LL1nod2 or } \\
\text { LHnod2 } \\
(10)\end{array}$ \\
\hline AA Tau & SLLL & 1.00 & 1.00 & 1.00 & 1.00 & 1.02 & 1.00 & 1.02 & 1.04 \\
\hline BP Tau & SLLL & 1.01 & 1.00 & 1.05 & 1.00 & 1.00 & 1.01 & 1.00 & 1.01 \\
\hline CI Tau & SLLL & 1.00 & 1.00 & 1.00 & 1.01 & 1.00 & 1.03 & 1.00 & 1.01 \\
\hline $\mathrm{CoKu} \mathrm{Tau/3}$ & SLLL & 1.01 & 1.00 & 1.00 & 1.00 & 0.96 & 1.00 & 1.00 & 1.00 \\
\hline $\mathrm{CoKu} \mathrm{Tau} / 4$ & SLLL & 1.01 & 1.00 & 1.00 & 1.01 & 1.00 & 1.00 & 1.00 & 1.01 \\
\hline CW Tau & SLSHLH & 1.13 & 1.14 & 1.10 & 1.09 & 1.25 & 1.00 & 1.00 & 1.00 \\
\hline CX Tau & SLLL & 1.12 & 1.00 & 1.24 & 1.20 & 1.21 & 1.20 & 1.20 & 1.21 \\
\hline CY Tau & SLLL & 1.01 & 1.00 & 1.00 & 1.01 & 1.01 & 1.00 & 1.00 & 1.00 \\
\hline DD Tau & SLLL & 1.00 & 1.00 & 1.01 & 1.00 & 1.00 & 1.01 & 1.00 & 1.00 \\
\hline DE Tau & SLLL & 1.00 & 1.00 & 1.00 & 1.00 & 1.00 & 1.00 & 1.00 & 1.01 \\
\hline DF Tau & SLSHLH & 1.00 & 1.00 & 1.00 & 1.00 & 1.17 & 0.96 & 1.02 & 1.00 \\
\hline DG Tau & SLSHLH & 1.00 & 1.00 & 1.05 & 1.00 & 1.12 & 1.00 & 1.05 & 1.06 \\
\hline DH Tau & SLLL & 1.00 & 1.00 & 1.00 & 1.00 & 1.00 & 1.01 & 1.00 & 1.03 \\
\hline DK Tau & SLSHLH & 1.01 & 1.00 & 1.00 & 1.01 & 1.00 & 1.01 & 1.00 & 1.00 \\
\hline DL Tau & SLSHLH & 1.01 & 1.00 & 1.00 & 1.00 & 1.00 & 1.04 & 1.00 & 1.01 \\
\hline DM Tau & SLLL & 1.15 & 1.15 & 1.15 & 1.15 & 1.00 & 1.00 & 1.00 & 1.01 \\
\hline DN Tau & SLLL & 1.00 & 1.00 & 1.00 & 1.00 & 1.02 & 1.00 & 1.00 & 1.01 \\
\hline DO Tau & SLSHLH & 1.01 & 1.00 & 1.00 & 1.00 & 1.00 & 1.07 & 1.05 & 1.06 \\
\hline DP Tau & SLLL & 1.00 & 1.00 & 1.01 & 1.00 & 1.01 & 1.00 & 1.00 & 1.01 \\
\hline DQ Tau & SLLL & 1.05 & 1.05 & 1.05 & 1.05 & 1.00 & 1.00 & 1.00 & 1.00 \\
\hline DR Tau & SLSHLH & 1.00 & 1.00 & 1.00 & 1.00 & 1.05 & 0.95 & 1.01 & 1.00 \\
\hline DS Tau & SLLL & 1.00 & 1.00 & 1.00 & 1.00 & 1.00 & 1.00 & 1.00 & 1.00 \\
\hline $\mathrm{F} 04147+2822$ & SLLL & 1.00 & 1.00 & 1.00 & 1.01 & 1.00 & 1.01 & 1.01 & 1.00 \\
\hline FM Tau & SLLL & 1.02 & 1.00 & 1.00 & 1.00 & 1.00 & 1.00 & 1.00 & 1.00 \\
\hline FN Tau & SLLL & 1.02 & 1.00 & 1.10 & 1.00 & 1.10 & 1.10 & 1.10 & 1.10 \\
\hline FO Tau & SLLL & 1.03 & 1.00 & 1.15 & 0.92 & 1.00 & 1.01 & 1.00 & 1.00 \\
\hline FP Tau & SLLL & 1.01 & 1.00 & 1.00 & 1.01 & 1.00 & 1.00 & 1.00 & 1.00 \\
\hline FQ Tau & SLLL & 1.00 & 1.01 & 1.00 & 1.00 & 1.01 & 1.00 & 1.00 & 1.00 \\
\hline FS Tau & SLSHLH & 1.00 & 1.00 & 1.02 & 1.00 & 1.09 & 0.93 & 1.00 & 1.03 \\
\hline FT Tau & SLLL & 1.00 & 1.00 & 1.00 & 1.00 & 1.00 & 1.00 & 1.00 & 1.00 \\
\hline FV Tau & SLSHLH & 1.01 & 1.00 & 1.04 & 1.00 & 1.12 & 0.97 & 1.00 & 1.00 \\
\hline FX Tau & SLLL & 1.01 & 1.00 & 1.02 & 1.00 & 1.00 & 1.00 & 1.05 & 1.06 \\
\hline FZ Tau & SLSHLH & 1.01 & 1.00 & 1.03 & 1.00 & 1.19 & 0.97 & 1.01 & 1.00 \\
\hline GG Tau A & SLLL & 1.04 & 1.03 & 1.04 & 1.03 & 1.00 & 1.00 & 1.00 & 1.00 \\
\hline GG Tau B & SLLL & 1.10 & 1.15 & 1.10 & 1.15 & 1.02 & 1.00 & 1.01 & 1.00 \\
\hline GH Tau & SLLL & 1.00 & 1.00 & 1.00 & 1.01 & 1.00 & 1.01 & 1.00 & 1.01 \\
\hline GI Tau & SLLL & 1.02 & 1.00 & 1.04 & 1.00 & 1.00 & 1.00 & 1.00 & 1.01 \\
\hline GK Tau & SLSHLH & 1.02 & 1.00 & 1.03 & 1.00 & 1.06 & 1.05 & 1.05 & 1.06 \\
\hline GM Aur & SLLL & 1.00 & 1.00 & 1.00 & 1.02 & 1.00 & 1.01 & 1.00 & 1.00 \\
\hline GN Tau & SLLL & 1.01 & 1.00 & 1.00 & 1.00 & 1.00 & 1.00 & 1.00 & 1.00 \\
\hline GO Tau & SLLL & 1.03 & 1.00 & 1.00 & 1.00 & 1.00 & 1.01 & 1.00 & 1.01 \\
\hline Haro 6-28 & SLSHLH & 1.03 & 1.00 & 1.00 & 1.00 & 1.00 & 1.00 & 1.00 & 1.00 \\
\hline Haro 6-37 & SLSHLH & 1.00 & 1.00 & 1.03 & 1.00 & 1.06 & 1.00 & 1.00 & 1.00 \\
\hline HK Tau & SLLL & 1.00 & 1.00 & 1.00 & 1.01 & 1.00 & 1.01 & 1.00 & 1.01 \\
\hline HN Tau & SLSHLH & 1.01 & 1.00 & 1.00 & 1.00 & 1.00 & 1.00 & 1.00 & 1.00 \\
\hline HO Tau & SLLL & 1.01 & 1.00 & 1.00 & 1.00 & 1.06 & 1.00 & 1.02 & 1.00 \\
\hline HP Tau & SLSHLH & 1.02 & 1.00 & 1.00 & 1.01 & 1.07 & 1.13 & 1.06 & 1.10 \\
\hline HQ Tau & SLSHLH & 1.01 & 1.00 & 1.00 & 1.01 & 1.00 & 1.11 & 1.03 & 1.03 \\
\hline IP Tau & SLLL & 1.01 & 1.00 & 1.01 & 1.00 & 1.00 & 1.00 & 1.00 & 1.01 \\
\hline IQ Tau & SLLL & 1.01 & 1.00 & 1.00 & 1.00 & 1.02 & 1.04 & 1.02 & 1.04 \\
\hline IS Tau & SLLL & 1.00 & 1.02 & 1.00 & 1.02 & 1.00 & 1.00 & 1.00 & 1.00 \\
\hline IT Tau & SLLL & 1.02 & 1.00 & 1.00 & 1.04 & 1.00 & 1.01 & 1.00 & 1.01 \\
\hline LkCa 15 & SLLL & 1.01 & 1.00 & 1.00 & 1.02 & 1.00 & 1.02 & 1.00 & 1.00 \\
\hline RW Aur A & SLSHLH & 1.00 & 1.00 & 1.00 & 1.00 & 1.01 & 1.00 & 1.01 & 1.00 \\
\hline UY Aur & SLSHLH & 1.00 & 1.00 & 1.00 & 1.00 & 1.11 & 1.01 & 1.08 & 1.07 \\
\hline UZ Tau E & SLSHLH & 1.03 & 1.00 & 1.00 & 1.02 & 1.37 & 0.87 & 1.10 & 1.11 \\
\hline V410 Anon 13 & SLLL & 1.02 & 1.00 & 1.00 & 1.02 & 1.08 & 1.00 & 1.05 & 1.00 \\
\hline V710 Tau & SLLL & 1.02 & 1.00 & 1.12 & 1.07 & 1.04 & 1.00 & 1.05 & 1.06 \\
\hline V807 Tau & SLLL & 1.01 & 1.00 & 1.00 & 1.01 & 1.01 & 1.00 & 1.00 & 1.00 \\
\hline V836 Tau & SLLL & 1.00 & 1.00 & 1.00 & 1.01 & 1.00 & 1.00 & 1.00 & 1.00 \\
\hline V955 Tau & SLLL & 1.00 & 1.00 & 1.00 & 1.01 & 1.00 & 1.00 & 1.00 & 1.01 \\
\hline VY Tau & SLLL & 1.00 & 1.00 & 1.01 & 1.00 & 1.01 & 1.00 & 1.00 & 1.01 \\
\hline XZ Tau & SLSHLH & 1.00 & 1.01 & 1.01 & 1.00 & 1.00 & 1.01 & 1.00 & 1.02 \\
\hline $\mathrm{ZZ} \mathrm{Tau}^{a}$ & SLLL & $\ldots$ & $\ldots$ & $\ldots$ & $\ldots$ & $\ldots$ & $\ldots$ & $\ldots$ & $\ldots$ \\
\hline ZZ Tau IRS & SLLL & 1.00 & 1.00 & 1.03 & 1.00 & 1.00 & 1.00 & 1.00 & 1.00 \\
\hline
\end{tabular}

Note. - Col. (1): Object name. Cols. (2)-(9): Multiplicative scalars applied to the spectrum of one order of one nod for a given object to match the flux density of the other nod as described in the text. SL2 means Short-Low order 2, SL1 is Short-Low order 1, LL2 is Long-Low order 2, LL1 is Long-Low order 1.

${ }^{a}$ For ZZ Tau, there were 6 DCEs per spectral order. Each DCE of each order was normalized to the DCE with the highest average flux density over that order, except for Long-Low Bonus order. In that case, the DCEs were normalized to the average flux level of the DCE with the second-highest flux density level (see discussion by Sargent et al. 2008). 